University of Tennessee Health Science Center

UTHSC Digital Commons

$12-2019$

\title{
The Discovery and Development of Thienopyrimidines as Inhibitors of Helicobacter pylori
}

\author{
Alex Kagabo Mugengana \\ University of Tennessee Health Science Center
}

Follow this and additional works at: https://dc.uthsc.edu/dissertations

Part of the Pharmacy and Pharmaceutical Sciences Commons

\section{Recommended Citation}

Mugengana, Alex Kagabo (https://orcid.org/0000-0002-2240-5928), "The Discovery and Development of Thienopyrimidines as Inhibitors of Helicobacter pylori" (2019). Theses and Dissertations (ETD). Paper 503. http://dx.doi.org/10.21007/etd.cghs.2019.0580.

This Dissertation is brought to you for free and open access by the College of Graduate Health Sciences at UTHSC Digital Commons. It has been accepted for inclusion in Theses and Dissertations (ETD) by an authorized administrator of UTHSC Digital Commons. For more information, please contact jwelch30@uthsc.edu. 


\title{
The Discovery and Development of Thienopyrimidines as Inhibitors of Helicobacter pylori
}

\author{
Abstract \\ The rate of successful treatment for Helicobacter pylori infections, with the clarithromycin triple therapy, \\ is only $75 \%$. The triple therapy, which consists of a proton pump inhibitor and two broad-spectrum \\ antibiotics such as clarithromycin and amoxicillin, is becoming less effective due to the rise of strains \\ with resistance against these antibiotics. In the search for narrow spectrum drugs for the treatment of $\mathrm{H}$. \\ pylori infections, a high-throughput screen was performed to identify selective compounds against $\mathrm{H}$. \\ pylori. This screen revealed two selective and structurally related thienopyrimidines. Structure-activity \\ relationship of the thienopyrimidines against $\mathrm{H}$. pylori was examined through the synthesis of 27 analogs, \\ efforts that merged elements of the two scaffolds. The resulting leads, including compound 25, \\ demonstrate high potency with an acceptable cytotoxicity profile against the human FaDu cells. Mode of \\ action studies were performed by the generation and sequencing of resistant mutants. These \\ experiments identified $\mathrm{H}$. pylori's respiratory Complex I as the putative target of the series, with the amino \\ acid changes found in the NuoD subunit. Lead compounds demonstrated efficacy in an ex vivo model but \\ not in the in vivo studies in a $\mathrm{H}$. pylori murine infection model, suggesting further optimization of the \\ pharmacological properties is required for this series. A scaffold hopping exercise was performed to \\ discover novel scaffolds with improved ADME properties. The pyrrolopyrimidine 56 emerged with good \\ anti-H. pylori activity, an improved solubility and is less bound to human plasma protein. Lead \\ optimization efforts are ongoing, to realize proof of concept of this series of compounds in the in vivo \\ efficacy studies. \\ Document Type \\ Dissertation \\ Degree Name \\ Doctor of Philosophy $(\mathrm{PhD})$ \\ Program \\ Pharmaceutical Sciences \\ Research Advisor \\ Richard E. Lee, Ph.D. \\ Keywords \\ Helicobacter pylori, Hit-to-lead optimization, Phenotypic high-throughput screen, Respiratory Complex I, \\ Structure-Activity Relationship, Thienopyrimidines \\ Subject Categories \\ Medicine and Health Sciences | Pharmacy and Pharmaceutical Sciences
}

This dissertation is available at UTHSC Digital Commons: https://dc.uthsc.edu/dissertations/503 
UNIVERSITY OF TENNESSEE HEALTH SCIENCE CENTER

DOCTOR OF PHILOSOPHY DisSERTATION

\section{The Discovery and Development of Thienopyrimidines as Inhibitors of Helicobacter pylori}

Author:

Alex K. Mugengana
Advisor:

Richard E. Lee, Ph.D.

A Dissertation Presented for The Graduate Studies Council of

The University of Tennessee Health Science Center

in Partial Fulfillment of the Requirements for the

Doctor of Philosophy degree from

The University of Tennessee

in

Pharmaceutical Sciences: Medicinal Chemistry

College of Graduate Health Sciences

December 2019 
Copyright (C) 2019 by Alex K. Mugengana.

All rights reserved. 


\section{DEDICATION}

To my parents, Gorrette and Celestin,

For their unconditional love and encouragement. 


\section{ACKNOWLEDGEMENTS}

I would like to thank my $\mathrm{PhD}$ advisor, Dr. Richard E. Lee for his support during my graduate studies.

I would like to thank the members of the Lee laboratory for their assistance during my training and the dissertation committee members, Dr. Isaac O. Donkor, Dr. Bernd Meibohm, Dr. Glen E. Palmer and Dr. Charles Rock, for their guidance in completing this dissertation.

I would like to acknowledge contributions from the laboratory of Dr. Mike LaFleur for testing the in vitro and in vivo activity of the compounds discussed in this dissertation and the members of the Lee laboratory, Nicole Vita and Elizabeth Griffith, for the computational work described in this dissertation. The in vitro pharmacokinetic properties of the lead compounds were determined and provided by the analytical core at St Jude Children's Research Hospital. The work described in this dissertation was supported by funds from the National Institutes of Health and ALSAC.

Last but not least, I would like to thank my parents and siblings for their care, and friends who were supportive during my training. 


\begin{abstract}
The rate of successful treatment for Helicobacter pylori infections, with the clarithromycin triple therapy, is only $75 \%$. The triple therapy, which consists of a proton pump inhibitor and two broad-spectrum antibiotics such as clarithromycin and amoxicillin, is becoming less effective due to the rise of strains with resistance against these antibiotics. In the search for narrow spectrum drugs for the treatment of H. pylori infections, a high-throughput screen was performed to identify selective compounds against $H$. pylori. This screen revealed two selective and structurally related thienopyrimidines. Structure-activity relationship of the thienopyrimidines against $H$. pylori was examined through the synthesis of 27 analogs, efforts that merged elements of the two scaffolds. The resulting leads, including compound 25, demonstrate high potency with an acceptable cytotoxicity profile against the human FaDu cells. Mode of action studies were performed by the generation and sequencing of resistant mutants. These experiments identified H. pylori's respiratory Complex I as the putative target of the series, with the amino acid changes found in the NuoD subunit. Lead compounds demonstrated efficacy in an ex vivo model but not in the in vivo studies in a H. pylori murine infection model, suggesting further optimization of the pharmacological properties is required for this series. A scaffold hopping exercise was performed to discover novel scaffolds with improved ADME properties. The pyrrolopyrimidine 56 emerged with good anti-H. pylori activity, an improved solubility and is less bound to human plasma protein. Lead optimization efforts are ongoing, to realize proof of concept of this series of compounds in the in vivo efficacy studies.
\end{abstract}




\section{TABLE OF CONTENTS}

CHAPTER 1. INTRODUCTION .....................................................................................1

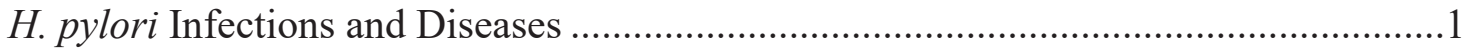

The Treatment Against H. pylori Infections ............................................................

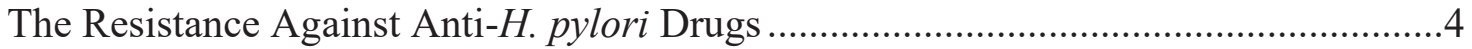

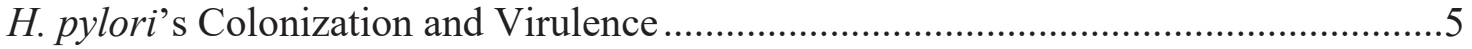

The Status of Discovery of the Anti-H. pylori Drugs...............................................

H. pylori Genome and Target-based Drug Discovery ...........................................

Phenotypic/Highthrough-put Screening for Anti-H. pylori Drugs ............................ 7

Examples of Novel Anti-H. pylori Compounds ................................................... 8

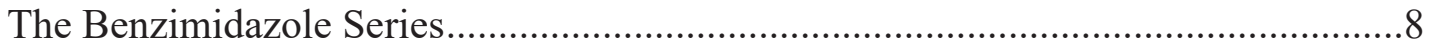

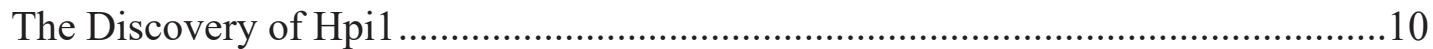

Repurposing Other Antibiotics for the Treatment of H. pylori............................... 10

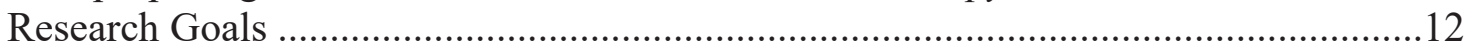

\section{CHAPTER 2. THE DISCOVERY AND DEVELOPMENT OF} THIENOPYRIMIDINES AS INHIBITORS OF HELICOBACTER PYLORI THROUGH INHIBITION OF THE RESPIRATORY COMPLEX I .....................13

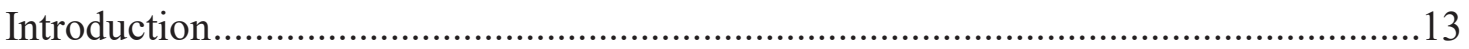

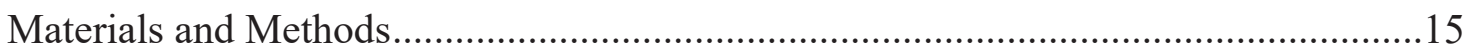

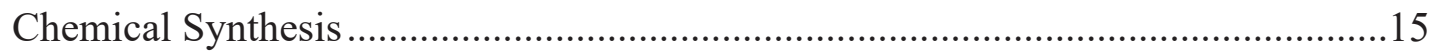

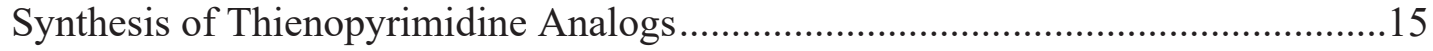

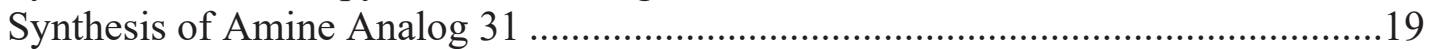

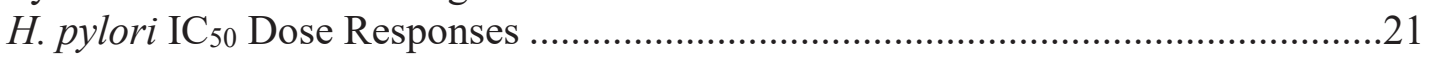

Cytotoxicity Assays ......................................................................................21

Generation of Resistant Mutants, Sequencing and Target Identification ..................22

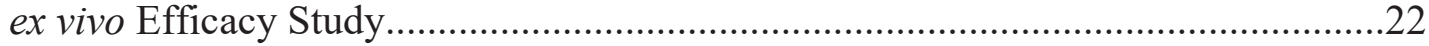

Homology Modeling of $H$. pylori's Complex I ...................................................23

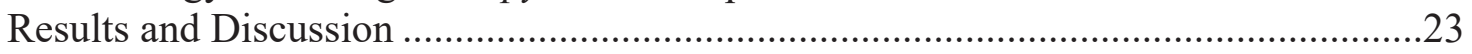

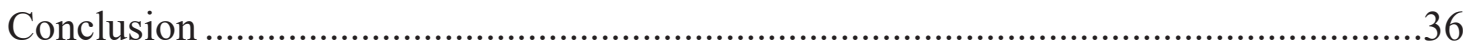

\section{CHAPTER 3. ADME OPTIMIZATION OF THE THIENOPYRIMIDINES}

AGAINST HELICOBACTER PYLORI .........................................................38

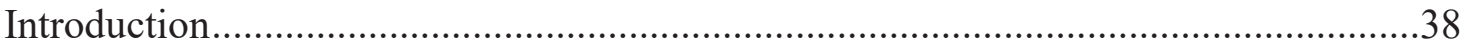

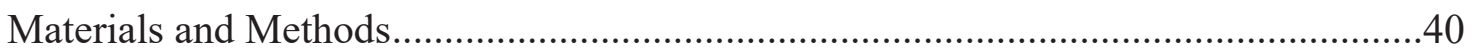

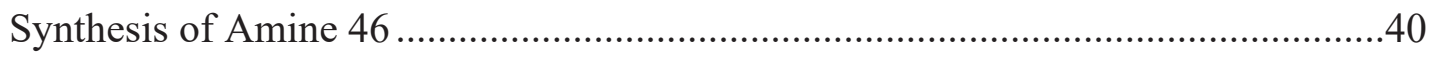

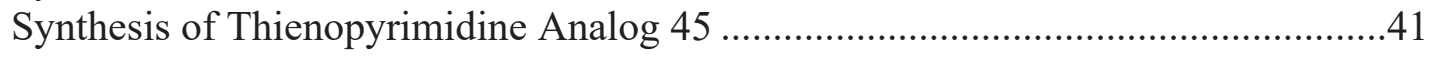

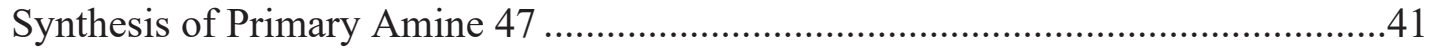

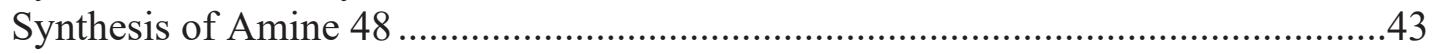

Synthesis of Thienopyrimidine Analog 49 .......................................................44

Synthesis of Thienopyrimidine and Pyrrolopyrimidine Analogs with

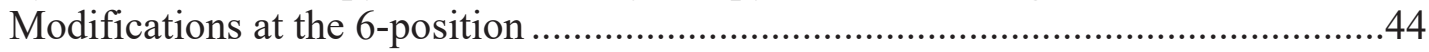

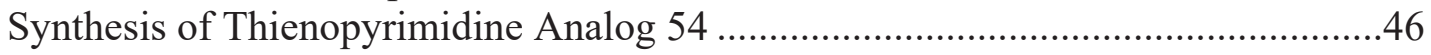

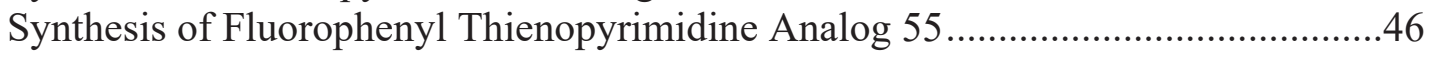


Synthesis of Pyrrolopyrimidine and Quinazoline Analogs with Modification at

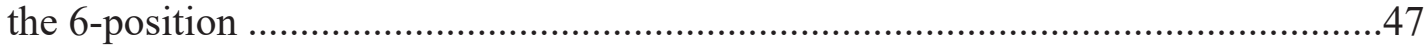

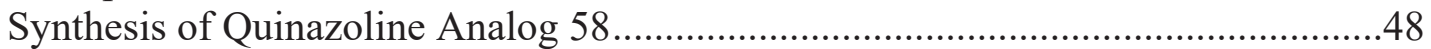

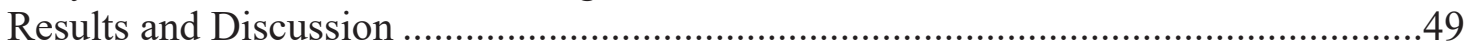

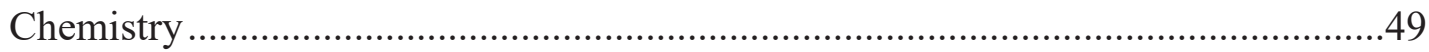

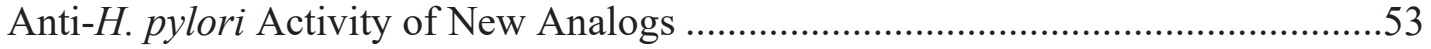

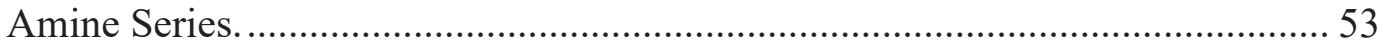

Heteroaryl Substitution. .......................................................................... 53

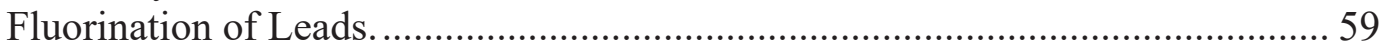

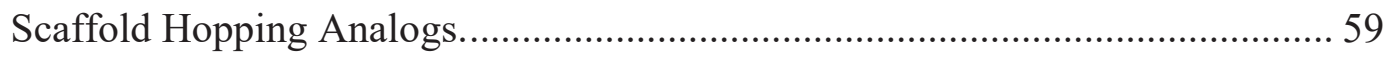

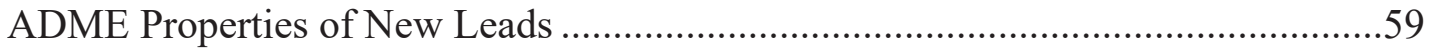

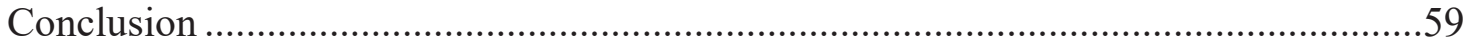

CHAPTER 4. CONCLUSION ..............................................................................66

LIST OF REFERENCES .........................................................................................66

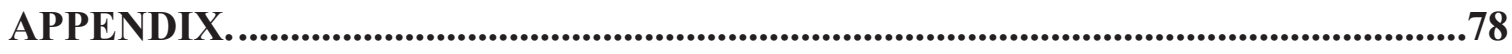

VITA 


\section{LIST OF TABLES}

Table 2-1. The structure-activity relationship of thienopyrimidines of series 1 ..........26

Table 2-2. The structure-activity relationship of thienopyrimidines of series 2 ..........27

Table 2-3. The structure-activity relationship of thienopyrimidines of series 3..........28

Table 2-4. The structure-activity relationship of series 4 with substitutions at the 5 and 6-positions of the thienopyrimdine core............................................32

Table 3-1. Thienopyrimidines with modified moieties at the 4-position for improved solubility and reduced lipophilicity. .......................................56

Table 3-2. Thienopyrimidines with modifications at the 4-position.............................58

Table 3-3. Pyrrolo[2,3-d]pyrimidine and quinazolines synthesized for improved

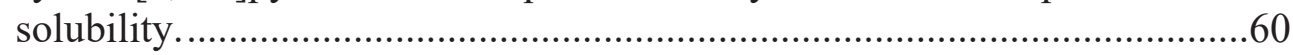

Table 3-4. ADME properties of selected compounds. .............................................61

Table A-1. Inhibition profile of thienopyrimidines against H. pylori ATCC 43504 strain, SS1 strain and SS1 mutant strain.

Table A-2. Inhibition profile of thienopyrimidines with modifications at the 5 and 6 positions against $H$. pylori ATCC 43504 strain, SS1 strain and SS1 mutant strain

Table A-3. ADME of the lead compount 25

Table A-4. Evaluation of in vivo pharmacokinetic properties of the lead compound 25 


\section{LIST OF FIGURES}

Figure 2-1. Structures of hit compounds from a highthrough-put screen against $H$. pylori and SAR development of the thienopyrimidines series.

Figure 2-2. Sequence alignment of the wildtype nuoD and two $H$. pylori mutant nuoD.

Figure 2-3. The homology model of H. pylori's respiratory Complex I, the putative target of the thienopyrimidines.

Figure 2-4. Compound 25 eradicates H. pylori in the ex vivo efficacy experiment. .......37

Figure 3-1. Lead compound 25 and targeted moieties to improve the ADME properties.

Figure A-1. In vivo efficacy of compound 25 in the $H$. pylori infected mouse model.....82 


\section{CHAPTER 1. INTRODUCTION}

\section{H. pylori Infections and Diseases}

Helicobacter pylori infects more than $50 \%$ of the world's population. ${ }^{1,2}$ In the United States, more than 500000 cases of $H$. pylori infections are diagnosed annually, according to the Center for Disease Control and Prevention. ${ }^{3}$ The majority H. pylori infections leads to gastritis, often without showing symptoms. ${ }^{1,4} 10 \%$ of the $\mathrm{H}$. pylori infections lead to peptic ulcers. ${ }^{5}$ Peptic ulcers are often chronic and may cause bleeding, perforation and eventually death. ${ }^{4}$ Less commonly, H. pylori infections may result into certain forms of cancer such as mucosa-associated lymphoid tissue (MALT) lymphoma, gastric and pancreatic adenocarcinoma. ${ }^{6}$ The World Health Organization (WHO) lists $H$. pylori as a class I carcinogen. ${ }^{7}$ Often, acute gastritis becomes chronic and then chronic gastritis leads to metaplasia and adenocarcinoma. ${ }^{4}$ Gastric cancer is the fourth most common cancer and the second leading cause of deaths among all cancers. ${ }^{8} \mathrm{H}$. pylori has also been implicated in the pathogenesis of many other diseases including idiopathic thrombocytopenia purpura, idiopathic iron deficiency anemia, ischaemic heart diseases, ischaemic cerebrovascular disease, atherosclerosis, Raynaud's phenomenon and many skin diseases. ${ }^{4}$

Barry Marshall and Robin Warren were awarded the Nobel Prize in 2005, for their work that established $H$. pylori as the causative agent of gastritis and peptic ulcers. ${ }^{9}$ Their discovery enlightened the long-standing ambiguity around the cause of peptic ulcers. As result, patients with peptic ulcers and gastric cancer are recommended to be tested for $\mathrm{H}$. pylori infections. ${ }^{10}$ The tests that are conducted to identify active infection include the urea breath test, fecal antigen test and mucosal biopsy-based testing that relies on endoscopy. ${ }^{11} \mathrm{H}$. pylori is generally identified in $70 \%$ of antral biopsy specimen of patients with chronic gastritis and $90 \%$ of patients with duodenal ulcers. ${ }^{4}$ The eradication of $\mathrm{H}$. pylori is recommended in treating gastritis, instead of drugs that suppress the high gastric acidity. ${ }^{12,13,14}$ Patients treated by eradicating the bacteria show lower relapse rate $(9 \%)$ without recurrence, as oppose to high relapse rates $(58-67 \%)$ identified with patients who manifest the infection after treatment. ${ }^{4} \mathrm{H}$. pylori eradication is also recommended in both treating low grade MALT lymphoma and preventing gastric cancer. ${ }^{15-17}$ According to a study that was conducted in Japan, nearly $80 \%$ of 420 patients who tested positive for the gastric MALT lymphoma, responded to the eradication therapy with minimal residual disease. ${ }^{18}$ Usually, the eradication of $H$. pylori is confirmed using the tests, mentioned above. ${ }^{11}$

\section{The Treatment Against $H$. pylori Infections}

The clarithromycin triple therapy consists of a Proton Pump Inhibitor (PPI), clarithromycin and either amoxicillin or metronidazole for 14 days. (Figure 1-1) In the 2017 American College of Gastroenterology (ACG) guidelines, the clarithromycin triple therapy is recommended as the first-line treatment for $\mathrm{H}$. pylori infections. ${ }^{11}$ However, 
A

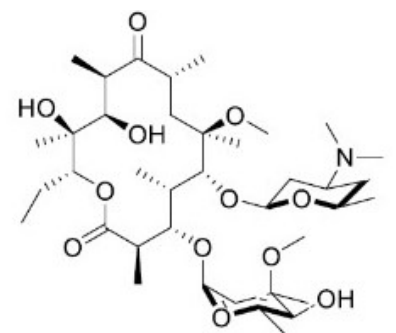

D

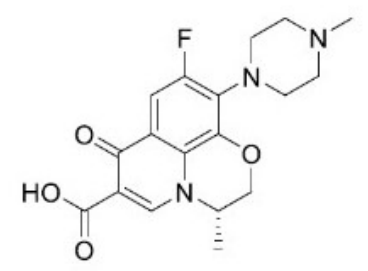

B
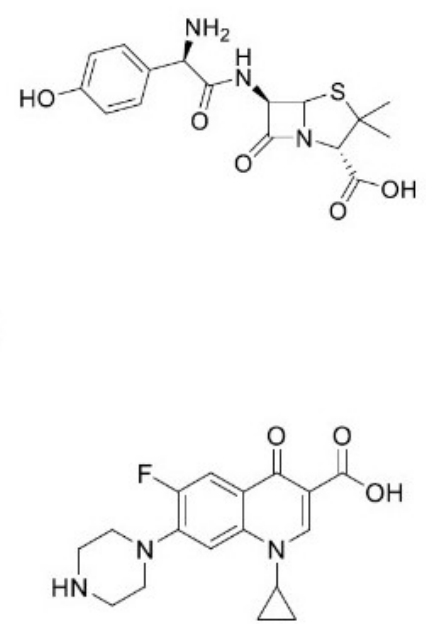

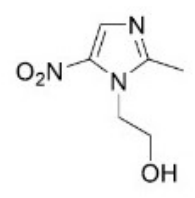

F

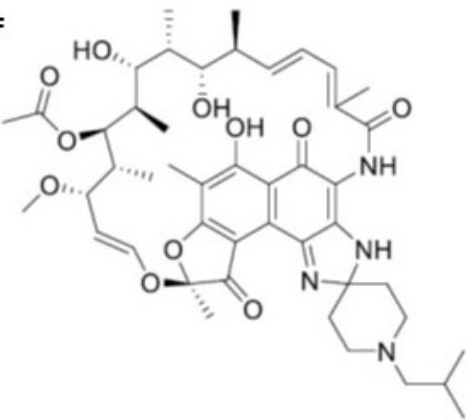

Figure 1-1. Anti-H. pylori drugs.

(A) Structure of clarithromycin. (B) Structure of amoxicillin. (C) Structure of metronidazole. (D) Structure of levofloxacin. (E) Structure of ciprofloxacin. (F) Structure of rifabutin. 
the clarithromycin triple therapy have shown limitations. The clarithromycin triple therapy has been associated with lower eradication rates, when it is administered for a shorter period of 7 to 10 days. The drug, clarithromycin is highly recommended as a first line treatment for patients who have not been previously exposed to macrolides and who are in the area identified with low resistance against clarithromycin $(<15 \%) .{ }^{19}$ Clarithromycin is a bacterial static agent that acts following the inhibition of protein synthesis via binding to the 50S ribosomal subunit. Amoxicillin is a bactericidal agent and inhibits the synthesis of the cell wall. These antibiotics act on actively replicating bacteria. ${ }^{20} \mathrm{~A}$ proton pump inhibitor such as omeprazole, acts by suppressing the secretion of gastric acid, which allow the bacteria to replicate and facilitate the bactericidal effects of the antibiotics.

A strong association has been reported between the number of previous exposures and a high risk of antibiotic resistance. ${ }^{21}$ In general, the appropriate regimen for the treatment against $H$. pylori infections is recommended based on the drug resistance pattern in the region and the patient's exposure to the antibiotics and the patient compliance. ${ }^{22}$ For patients who have an allergy to penicillin, amoxicillin is substituted with metronidazole in the clarithromycin triple therapy regimen. The use of either metronidazole or amoxicillin achieves the same therapeutic effect. ${ }^{23}$ Inside the bacteria, metronidazole is metabolized by nitroreductase enzymes into metabolites that are toxic to bacteria. The triple therapy regimen, containing metronidazole, is recommended to patients who have been previously exposed to macrolides.

The bismuth quadruple therapy is another recommended first-line treatment, which consists of a PPI, bismuth, a tetracycline and a nitroimidazole for 10-14 days. ${ }^{11}$ (Figure 1-1) In case the clarithromycin triple therapy fails to eradicate the infection, the bismuth quadruple therapy is recommended as a salvage treatment. The use of bismuth as part of the regimen achieves the eradication rate of above $90 \% .{ }^{24}$ However, the role of bismuth is not well understood. Marcus et al. suggests that bismuth hinders proton entry into the bacteria which leads to the up-regulation of growth depended genes and H. pylori becomes more susceptible to the antibiotics. ${ }^{25}$

The concomitant, the sequential, the hybrid and the fluoroquinolone sequential therapies are other first line treatment options that are used worldwide for the treatment of H. pylori infection. The concomitant therapy consists of a PPI, clarithromycin, amoxicillin and a nitroimidazole for 10 to 14 days. ${ }^{11}$ The sequential therapy consists of a PPI and amoxicillin for 5 to 7 days followed by a PPI, amoxicillin, clarithromycin and a nitroimidazole for 5 to 7 days. The hybrid therapy consists of a PPI and amoxicillin for 7 days followed by a PPI, amoxicillin, clarithromycin and a nitroimidazole for 7 days. Fluoroquinolone sequential therapy consists of a PPI and amoxicillin for 5-7 days followed by a PPI, fluoroquinolone and nitroimidazole for 5-7 days with levofloxacin and ciprofloxacin as the fluoroquinolone drugs. (Figure 1-1) The concomitant, the sequential and the hybrid therapies have been found to achieve the same efficacy based on clinical research data published by ACG. ${ }^{11}$ According to the recent meta-analysis, the sequential and concomitant therapies have shown higher eradication rates ( $>90 \%)$, than the clarithromycin triple therapy $(>70 \%) .{ }^{10}$ The concomitant therapy, in particular, is 
preferred among these alternative first-line therapies, given it constitutes a simpler regimen that affords patient compliance.

The levofloxacin triple therapy, the rifabutin triple therapy and the high-dose dual therapy are recommended as salvage treatments, in case the first line treatment fails to eradicate the infections. (Figure 1-1) The levofloxacin triple therapy, which consists of a Proton Pump Inhibitor (PPI), levofloxacin and amoxicillin for 10 to 14 days, is recommended as a salvage regimen, in case the clarithromycin triple therapy or the bismuth quadruple therapy fails to eradicate $H$. pylori. Rifabutin triple regimen consists of a PPI, amoxicillin and rifabutin for 10 days. High-dose dual therapy consists of a PPI and amoxicillin administered for 14 days. Despite the availability of these alternative regimens, the rate of successful treatments has significantly declined worldwide, due to the rise of resistance against the antibiotic components of these regimens. ${ }^{1,26}$

\section{The Resistance Against Anti-H. pylori Drugs}

H. pylori has become a global threat due to a steady increase of worldwide drug resistance against anti- $H$. pylori drugs. $H$. pylori is listed as a high priority drug-resistant bacterium by the World Health Organization among bacteria that are threat to the human health. ${ }^{7}$ Worth noting, the rate of successful treatment of H. pylori infections has dropped below $80 \%{ }^{1,26}$ In a recent study, $22 \%$ of patients tested in a clinical trial were not cured by either the triple therapy or the quadruple therapy. ${ }^{27}$ In the early 1990 s, the worldwide treatment rate of $H$. pylori infections with the triple therapy were recorded at $80-90 \%{ }^{28}$ From 1998 to 2002, the United States national sampling study reported a resistance rate of $21 \%$ against metronidazole and $13 \%$ against clarithromycin. ${ }^{29}$ Between the year of 2000 and 2008, the resistance rate in Alaska was recorded at 42\% and 30\% against metronidazole and clarithromycin respectively. ${ }^{30}$ In 2012, the resistance rates were reported at $20 \%$ and $16 \%$ by the Houston VA medical center, for metronidazole and clarithromycin respectively. ${ }^{31}$ In particular, the resistance against metronidazole has been fueled by its overuse in the regions predominated by parasitic diseases. ${ }^{19}$ In certain parts of the world, such as Japan, the resistance rate against metronidazole is low, due to its restricted use at the national level. ${ }^{32}$

The effectiveness of the regimens for the treatment of $H$. pylori infection, has declined due to the resistance against the antibiotics at various degrees. The metaanalysis studies have revealed that the resistance against clarithromycin is the major contributor to the failure of the triple therapy. ${ }^{10}$ More worrisome, the fluoroquinolone resistance has been recorded as high as clarithromycin resistance (rate: 16\%), in North America. ${ }^{31,33}$ Although in vitro resistance against metronidazole (rate: $20 \%$ ) is high worldwide, $H$. pylori mutations against clarithromycin or levofloxacin are the most clinically relevant mutations. They are usually identified using molecular methods including polymerase chain reaction or fluorescently-labeled nucleic acid hybridization. These methods have been optimized overtime and are simply applied to clinical isolates collected from gastric mucosal biopsies or fecal samples. ${ }^{34}$ The resistance against amoxicillin, tetracycline and rifabutin are still low (rate: $<2 \%$ for each), whereas the 
resistance against bismuth hasn't been reported worldwide. ${ }^{11,35}$ The resistance rates against these antibiotics are registered higher in children than adults, because the same drugs are prescribed for the treatment of diseases that are prevalent in children, such as the respiratory and parasitic diseases. ${ }^{36}$ Clarithromycin, fluoroquinolones and rifabutin are generally discouraged to be reused in the clinic, given the resistance against these drugs cannot be overcome by simply increasing the dose.

Point mutations have been the primarily mechanism of resistance against the antibiotics for the treatment of $H$. pylori infections. In $H$. pylori, point mutation in the 23S rRNA are the main mechanism of resistance against clarithromycin. Mutations in the penicillin binding proteins drive the resistance against amoxicillin. The resistance against levofloxacin comes from the mutation in the gyrA gene, which encodes the subunit A of DNA gyrase. ${ }^{38}$ Various mechanisms have been reported as the basis for the resistance against metronidazole.

The resistance against metronidazole originate from the mutations in the $\mathrm{rdxA}$ gene. In bacteria, rdxA encodes for NADPH nitroreductase, which is responsible for activating metronidazole and other nitroaromatic compounds into toxic metabolites. ${ }^{39}$ The efflux pump, hefA is found overexpressed in resistant $H$. pylori strains and has been reported to play a key role in reducing the cellular concentrations of metronidazole. ${ }^{40}$ hefC, a multidrug efflux pump, also reduces drug concentrations to below effective concentrations. $^{41}$

The side effects from the use combination broad spectrum antibiotics, contributes to poor patient compliance affecting the success of these treatments. ${ }^{10}$ The use of these broad-spectrum antibiotics perturbs the gut microbiome, leading to symptoms like diarrhea. ${ }^{11}$ Other side effects associated with the triple therapy include: headache, dizziness, nausea, dysgeusia, dyspepsia, pseudomembranous colitis, mycosis, sore mouth and tongue, drug hypersensitivity and paraesthesia. Given the rise of resistance, specific therapies against $H$. pylori infections are needed to preserve these broad- spectrum drugs, that are crucial for the treatment of other infections and to mitigate the side effects associated with these drugs.

\section{H. pylori's Colonization and Virulence}

H. pylori is a spiral gram-negative bacterium that colonizes in the human stomach. ${ }^{42} H$. pylori infections are generally acquired at a young age via oral-oral route. ${ }^{43,44,45}$ Given $H$. pylori can only survive for 30 minutes, $H$. pylori must move quickly to avoid gastric emptying and penetrate the stomach mucus layer. ${ }^{46} \mathrm{H}$. pylori is found in the corpus and the antrum regions of the stomach, which differ in their cellular make up. ${ }^{47} H$. pylori colonizes areas in close association to the epithelial cells, to form a niche where it releases cytotoxins. ${ }^{48}$ Although, the majority of bacteria are found freely swimming in the lumen, important populations of $\mathrm{H}$. pylori are also found beneath the gastric mucous layer and within the glands, regions that are considered more favorable environment for long term survival than the lumen $(\mathrm{pH}<2)$. 
The $\mathrm{pH}$ gradient of different regions of the stomach is a major factor in determining $H$. pylori's colonization site. ${ }^{46} \mathrm{H}$. pylori is a bioenergetically neutrophil, with the ability to survive in an environment with a $\mathrm{pH}$ range of $4-8 .{ }^{49}$ Unlike other bacteria, $H$. pylori can inhabit acidic milieu, by maintaining a favorable $\mathrm{pH}$ in the proximity via 2 proteins: the urease which hydrolyzes urea into $\mathrm{CO}_{2}$ and ammonia, and the $\alpha$-carbonic anhydrase protein, which catalyzes the conversion of $\mathrm{CO}_{2}$ to $\mathrm{HCO}_{3}{ }^{-}$and eventually reduces the concentrations of $\mathrm{HCl}^{50-52}$ UreI is the proton gated channel, responsible for the urea influx. The protein urease is also found at the surface layer of the cell. The ammonia reacts with $\mathrm{HCl}$ to create a neutral surrounding environment in the periplasm and around the bacterium. The diffusion rate of chemicals, including ammonia, is significantly diminished in the mucus, which allows the acid to be neutralized and the neutral $\mathrm{pH}$ is maintained for a long period. ${ }^{53}$

H. pylori reaches the epithelial cells at the surface of the stomach, by motility with the flagella and the chemotaxis signaling system. ${ }^{54}$ Recent studies have shown that the wildtype strains swim faster in viscous environment than the mutants with straight cell bodies, suggesting $H$. pylori's spiral shape facilitate the bacteria to penetrate the mucus, in addition to the flagella. ${ }^{55,56}$ The bacterium utilizes chemotaxis, a mechanism that allows $H$. pylori to measure the concentration of the attractant and repellent substances, to flee undesirable conditions for growth. With chemotaxis, H. pylori can detect urea, metals and amino acids in the environment. ${ }^{57-59}$ It can detect injured epithelial cells that supply high concentrations of nutrients in the environment. ${ }^{60}$ The antrum and the corpus are populated with differentiated cells, with variation in concentrations of nutrients and metabolites such as sugars and amino acids. H. pylori's chemoreceptors, known as transducer like proteins, TlpA, TlpB, TlpC and TlpD, play an important role of inputting environment signals that the bacterium utilizes to determine the swimming direction toward a favorable environment to grow and colonize. ${ }^{61}$ For instance, H. pylori's TlpA senses its attractant, arginine, and in response the bacterium swims toward the source of this essential amino acid. ${ }^{62} \mathrm{TlpB}$ senses a repellent, autoinducer 2 (AI-2), that allows H. pylori to determine the density of other bacteria in the surrounding area as it grows and colonizes in the stomach. ${ }^{63}$

H. pylori produces two main cytotoxins: the cytotoxic associated gene (CagA) and the vacuolating cytotoxin (VacA) ${ }^{64,65} \mathrm{H}$. pylori adheres to the epithelial cells using adhesion factors to deliver CagA. ${ }^{66,67}$ Type IV secretion system (T4SS) delivers CagA upon attachment to the epithelial cells. Inside the human cells, CagA triggers the alteration of transduction signals and gene expression. ${ }^{68-70}$ As a result, different signaling pathways are affected, including pathways that participate in cell-cell tight junction and pro-inflammatory response. Upon interaction with the host cell, T4SS alone can trigger pro- inflammatory response and the production of the chemokine IL- $8 .{ }^{71}$ Unlike CagA, H. pylori actively secretes VacA not requiring adhesion which interacts with the plasma membrane of host cells, leading to the formation of anion channels, $\mathrm{Cl}^{-}$ions influx and the formation of vacuoles. ${ }^{72}$ Inside the host cell, VacA leads to the release of proinflammatory proteins and the induction of apoptosis. ${ }^{73,74} \mathrm{H}$. pylori benefits from 
apoptosis of the host cells, which results in an increase of nutrients in the surrounding environment or diminished immunity that comes with the degradation of immune cells. ${ }^{75}$

\section{The Status of Discovery of the Anti-H. pylori Drugs}

\section{H. pylori Genome and Target-based Drug Discovery}

Advances in whole genome sequencing have provided opportunities for targetbased drug discovery against $H$. pylori. Targeting the genes that promotes the growth and colonization of $\mathrm{H}$. pylori is a promising strategy in the discovery of new therapies against H. pylori infections. In 1997, the complete genome sequence of the H. pylori 26695 strain became available. ${ }^{77}$ A couple of years later, the genome of H. pylori J99 strain was reported. ${ }^{78}$ The analysis of these genomes revealed that $60 \%$ of the genes produce proteins of known function based on the sequence similarity to their orthologs that are found in the public database. ${ }^{79} 23 \%$ of the remaining genes have their corresponding orthologs in the public domain, however the function of their gene products is unknown. The rest of the genes (17\%) have not been matched in the public database and the function of these genes have yet to be determined.

A recent transposon mutagenesis study identified $344 \mathrm{H}$. pylori essential genes. ${ }^{80}$ The function of $24 \%$ of these $H$. pylori specific genes remains unknown. Some of these unique $H$. pylori genes are believed to enable $H$. pylori, to grow under the gastric mucosa, in close association with the epithelial cells. ${ }^{81}$ Other studies have discovered genes responsible for the motility of $\mathrm{H}$. pylori, a function that facilitates $\mathrm{H}$. pylori to penetrate and colonize in the mucus. ${ }^{82}$ Other genes have been associated with chemotaxis, a mechanism that enables $H$. pylori to survive the gastric acidity. ${ }^{83,84}$ More genes have identified by the knock out mutagenesis to be involved in different cellular functions such as cell envelope synthesis, cell division, protein synthesis, gene expression and regulation, cell metabolism and energetics. ${ }^{79}$ The existence of these $H$. pylori specific genes suggest that $H$. pylori possesses unique proteins that can be targeted for the treatment of H. pylori.

\section{Phenotypic/Highthrough-put Screening for Anti-H. pylori Drugs}

The existence of many unique genes provides opportunities to selectively target H. pylori. The highthrough-put screen of compound library is a promising approach to identify active compounds against $H$. pylori. In addition, there is a high likelihood of finding selective hits against $H$. pylori by screening commercial libraries, given these libraries are often screened to identify broad-spectrum, while narrow-spectrum compounds are overlooked. 


\section{Examples of Novel Anti-H. pylori Compounds}

Various research groups have published compounds that have demonstrated activity and selectivity against $H$. pylori. Katsura et al. published a series of guanidino analogs that demonstrates selectivity comparable to the standard of care drugs such as metronidazole and amoxicillin. ${ }^{85-89}$ The structure activity relationship of the guanidino compounds focused on the guanidine substitution and the 4 position of the thiazole ring. ${ }^{86,88-90}$ Among these guanidino analogs, compound 6 (Figure 1-2A) demonstrates the highest potency with an MIC of $0.17 \mathrm{ug} / \mathrm{mL} .{ }^{85}$ Ando et. al reported a series of arylacetamides analogs that were discovered through compound screening efforts. ${ }^{91}$ The lead compound, structure shown in Figure 1-2G, demonstrates high potency (MIC $=0.39$ $\mu \mathrm{g} / \mathrm{mL}$ ), selectivity and stability in acidic conditions. The pyrazole derivatives have demonstrated activity and selectivity against $H$. pylori (Figure 1-2H) demonstrates good potency $(\mathrm{MIC}=0.125 \mu \mathrm{g} / \mathrm{mL})$ and selectivity against $H$. pylori. ${ }^{92-94}$ Dihydroorotate dehydrogenase (DHOase) was reported as the target of the pyrazole lead compound (MIC $=0.125 \mu \mathrm{g} / \mathrm{mL}$, Figure 1-2H), a key enzyme in the de novo biosynthesis of pyrimidines. Other natural product compounds have demonstrated activity and selectivity against $H$. pylori, including the pyloricidins (A, B and C), the phthalide derivatives, the pyrone derivatives, Indolmycin and Calvatic acid. ${ }^{95-104}$ These compounds, structures shown in Figure 1-2B, C, D, E and F, have demonstrated potency with a MIC equivalent to 0.006 $\mu \mathrm{g} / \mathrm{mL}, 5 \mathrm{ng} / \mathrm{mL}$ and $0.003 \mu \mathrm{g} / \mathrm{mL}, 0.016 \mu \mathrm{g} / \mathrm{mL}$ and $0.016 \mu \mathrm{g} / \mathrm{mL}$ respectively.

\section{The Benzimidazole Series}

Researchers at AstraZeneca discovered a series of benzimidazole analogs including lead compound in Figure 1-2I with a high potency (MICs $<0.5 \mathrm{ug} / \mathrm{mL}$ ) and selectivity against diverse $H$. pylori strains. ${ }^{105}$ In this study, resistant $H$. pylori mutants were generated with the lead compound of the series (Figure 1-2I). ${ }^{106}$ The genomic DNA of the mutants were isolated and transformed into a sensitive strain (AH244), confirming that these DNAs can confer resistance. The genomic library of the mutant was constructed and mapped onto the $H$. pylori strain J99 genome to identify resistant genes. This study revealed a single point mutation in nuoD (NADH:Quinone oxidoreductase) that yielded the amino acid change G398S. NuoD was confirmed as the putative target, with the additional 32 independent spontaneous mutants that revealed missense mutations which resulted in the amino acid changes: G398S, F404S and V407M. NuoD is one of the polypeptides that make up H. pylori's respiratory Complex I, a proton-pumping oxidoreductase that is located in the plasma membrane. ${ }^{107}$ It catalyzes the first step of oxidative phosphorylation, by transferring 2 electrons from NADH to quinone.

According to this study, the benzimidazoles bind at the interface of NuoD and the neighboring subunit NuoB. H. pylori's complex I and E. coli's complex I were sensitive to rotenone, an inhibitor of Rhodobacter capsulatus' complex I inhibitor, whereas the $E$. coli's complex I was not sensitive to the benzimidazole lead (Figure 1-2I), suggesting that the benzimidazoles are selective against $H$. pylori. ${ }^{108}$ Knockout mutagenesis experiments determined that nuoD was essential for the viability of $H$. pylori strain J99 
A

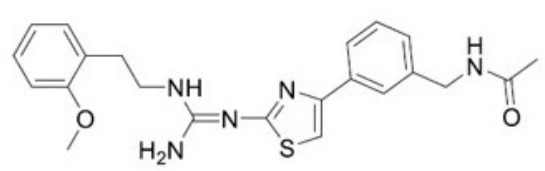

C

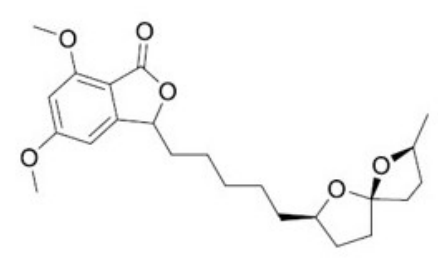

E

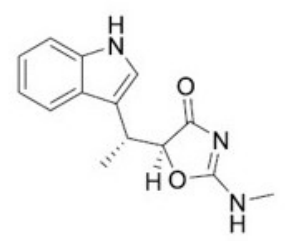

G<smiles>CNC(=O)c1cccc(NC(=O)Cc2cccc(Br)c2)c1</smiles>

I

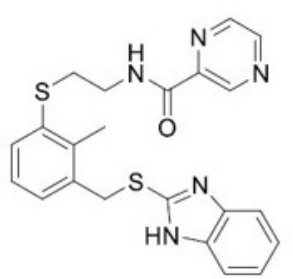

B<smiles>C=CC[C@H](N)C(=O)N[C@H](CO)[C@H](O)[C@H](O)C(O)C(=O)N[C@H](CC(=O)O)c1ccccc1</smiles>

D

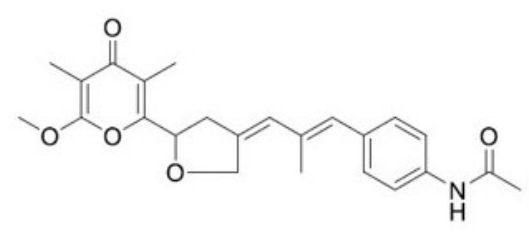

F

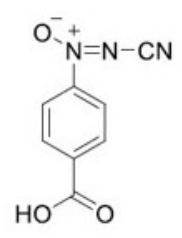

H

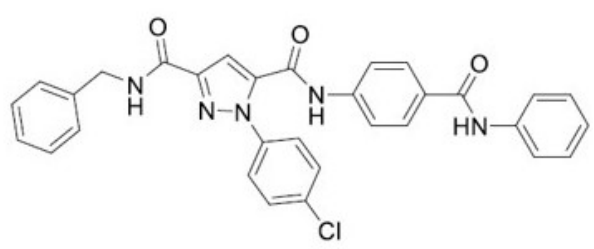

J

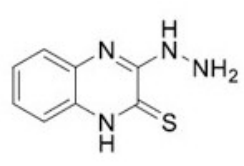

Figure 1-2. Structures of published anti-H. pylori compounds.

(A) Guanidinothiazole derivative ( $\mathrm{MIC}=0.17 \mu \mathrm{g} / \mathrm{mL}$ ). (B) Pyloricidin derivative ( $\mathrm{MIC}<$ $0.006 \mu \mathrm{g} / \mathrm{mL}$ ). (C) Phthalide derivative ( $\mathrm{MIC}=5.00 \mathrm{ng} / \mathrm{mL})$. (D) Pyrone derivative (MIC $=0.003 \mu \mathrm{g} / \mathrm{mL})$. (E) Indolmycin $(\mathrm{MIC}=0.016 \mu \mathrm{g} / \mathrm{mL})$. (F) Calvatic acid $(\mathrm{MIC}=0.016$ $\mu \mathrm{g} / \mathrm{mL}) .(\mathrm{G})$ Arylacetamide derivative $(\mathrm{MIC}=0.39 \mu \mathrm{g} / \mathrm{mL})$. $(\mathrm{H})$ Pyrazole derivative $(\mathrm{MIC}=0.125 \mu \mathrm{g} / \mathrm{mL})$. (I) Benzimidazole derivative ( $\mathrm{MIC}<0.5 \mu \mathrm{g} / \mathrm{mL})$. (J) HPi1 ( MIC $<0.16 \mu \mathrm{g} / \mathrm{mL})$. 
and AH244. In E. coli, nuoD and nuoC genes are found fused and they encode a single polypeptide. ${ }^{109}$ In $H$. pylori, these genes are separated, emphasizing the essentiality of nuoD in $H$. pylori, and not for other bacteria including E. coli.

\section{The Discovery of Hpi1}

Recently, we conducted a highthrough-put screen of 30,000 compounds to identify selective compounds with activity against $H$. pylori. ${ }^{81}$ The conditions for growing H. pylori were optimized in a 384 well plate, a challenging foot given the requirement of H. pylori for microaerophilic growth conditions. Promiscuous compounds were identified and eliminated studying the activity against a panel of commensal bacteria.

Bacterial growth was measured by reading the reduction of resazurin (blue dye), a metabolic indicator, to resorufin (pink fluorescent compound). The screen yielded HPi1 (Figure 1-2J), a selective hit compound, which demonstrates a high in vitro potency ( $\mathrm{IC}_{50}$ of $\left.0.24 \mu \mathrm{M}\right)$ compared to Clarithromycin $(0.04 \mu \mathrm{M})$ and good selectivity against a panel of commensal bacteria such as Lactobacillus casei, Lactobacillus reuteri and Bifidocterium longum. In the in vivo studies, HPil showed efficacy when tested in the $H$. pylori murine model at $25 \mathrm{mg} / \mathrm{kg}$ (p.o.). Hpil reduces the colony counts to below the limit of detection. Attempts to generate resistant mutants against HPi1, by serial passage on agar plate or from Hpil treated mice, were not successful and its target is unknown. ${ }^{53}$

\section{Repurposing Other Antibiotics for the Treatment of $\boldsymbol{H}$. pylori}

Fluoroquinolone compounds have been explored for the eradication of H. pylori. Finafloxacin, a novel fluoroquinolone, demonstrates superior anti-H.pylori activity in acidic conditions than other fluoroquinolones. ${ }^{110}$ Sitafloxacin, a fluoroquinolone antibiotic, show higher anti-H. pylori activity $\left(\mathrm{MIC}_{90}: 0.06 \mu \mathrm{g} / \mathrm{mL}\right)$ than metronidazole, clarithromycin, amoxicillin, tetracycline, doxycycline, minocycline, norfloxacin, ciprofloxacin and levofloxacin against $293 \mathrm{H}$. pylori strains, with low resistance development. ${ }^{111}$ In combination with lansoprazole, sitafloxacin eradicates $H$. pylori in vivo at $83.3 \%$ rate. At $1 \mathrm{mg} / \mathrm{kg}$, the Cmax of sitafloxacin is 10 fold higher than its MIC. DX-619, a des-F(6)-quinolone, is active against gram-positive bacteria including MRSA and vancomycin resistant enterococci. ${ }^{112}$ DX-619 also demonstrates bactericidal activity against $293 \mathrm{H}$. pylori strains, including those that are resistant to metronidazole, clarithromycin, amoxicillin and levofloxacin, with $\mathrm{MIC}_{50}$ of $0.008 \mathrm{mg} / \mathrm{L}$ and $\mathrm{MIC}_{90}$ of $0.06 \mathrm{mg} / \mathrm{L}$.

Some macrolides have been reported to show anti-H. pylori activity. Azithromycin, a macrolide antibiotic, demonstrates an in vitro $\mathrm{MIC}_{90}$ of $0.25 \mathrm{mg} / \mathrm{L} .{ }^{113}$ Azithromycin is stable in acidic conditions and exhibits physical chemical properties that allows a rapid migration of drug from blood to tissue and a slow release from tissue to blood circulation. The evaluation of in vivo pharmacokinetic properties revealed that the concentration of Azithromycin in mucosa was significantly higher than in plasma, 
ranging between $18.5 \mathrm{mg} / \mathrm{kg}$ and $24.6 \mathrm{mg} / \mathrm{kg}$ after a single oral administration (2.3-4.6 $\mathrm{mg} / \mathrm{kg}$ ). After 30 days of treatment with azithromycin at $500 \mathrm{mg} / \mathrm{kg}$ (+ omeprazole at 40 $\mathrm{mg} / \mathrm{kg}$ ), eradication of $H$. pylori was achieved in $80 \%$ of patients. Dirithromycin and roxithromycin demonstrates anti- $H$. pylori activity with $\mathrm{MIC}_{50}$ of 0.25 and $<0.008$ $\mu \mathrm{g} / \mathrm{mL}$, respectively. ${ }^{114}$ These macrolides show acid stability unlike other macrolides such as erythromycin, ethylsuccinate, josamycin and spiramycin.

Marketed drugs to treat infections caused by parasites, have also investigated for growth inhibition of $H$. pylori. Artemisinin and its derivatives have been explored for their anti-H. pylori activity. ${ }^{115}$ Five artemisinin derivatives, b-artecyclopropylmether, barteether, a-arteether, b-artemether and b-artefurfurylether, show anti-H. pylori activity and lack activity against the majority of other bacterial and fungal strains. Bartecyclopropylmether is the most active with MIC of $0.25-1 \mu \mathrm{g} / \mathrm{mL}$ at the stomach $\mathrm{pH}$. B-artecyclopropylmether demonstrates mild in vivo efficacy at $50 \mathrm{mg} / \mathrm{kg} /$ day as a single therapy. The mechanism of action of the artemisinin derivatives is not well understood. Nitazoxanide (NTZ), a thiazolide drug, is a broad-spectrum drug used for the treatment of infections caused by parasites and helminths. ${ }^{116} \mathrm{NTZ}$ exhibits activity against $H$. pylori and is a non-competitive inhibitor of pyruvate:ferredoxin/flavodoxin oxidoreductase (PFOR) of H. pylori. PFOR catalyzes the oxidative decarboxylation of pyruvate to acetyl $\mathrm{CoA}$ and $\mathrm{CO}_{2}$. It is only found in anaerobic bacteria, anaerobic parasites and epsilon proteobacteria.

Drugs targeting proton pump enzymes have demonstrated anti-H. pylori activity. Rabeprazole and its thioether derivative inhibits the motility of $H$. pylori with inhibition of $50 \%$ of motility at 16 and $0.25 \mu \mathrm{g} / \mathrm{mL}$ respectively. ${ }^{117}$ The inhibition of motility is observed instantly when bacteria is exposed to the agent, but growth inhibition is realized after hours of incubation. Lansoprazole, a benzimidazole proton pump inhibitor, show high activity against $H$. pylori with MIC of 3.13-12.5 $\mu \mathrm{g} / \mathrm{mL} .{ }^{118}$ Under acidic conditions, lansoprazole is transformed into AG-2000 and AG-1812, which show comparable activity to lansoprazole. These compounds lead to morphological change of cell, their constriction, collapse of cell surface structures and eventually cell death. Lansoprazole and its metabolites (AG-2000 and AG-1812) do not show activity at $100 \mu \mathrm{g} / \mathrm{mL}$ against aerobic, anaerobic bacteria and campylobacter jejuni. Lansoprazole is found highly distributed in the gastric mucosa and less in the serum. Idebenone and other quinones including duroquinone, menadione, juglone and coenzyme q1 inhibit $H$. pylori growth at the $\mathrm{MIC}_{90}$ of $1.6-3.2 \mu \mathrm{g} / \mathrm{mL} .{ }^{119}$ Idebenone inhibits respiration and triggers the reduction of the cellular ATP level, without showing adverse effect to human cells. Idebenone is selective against $H$. pylori and does not show activity against a variety of bacteria including Bacillus subtilis, Staphylococcus aureaus, Streptococcus salivarius and Pseudomonas aeruginosa.

Other antibiotics and non-antibiotics have shown anti-H. pylori activity. Blactams including cefuroxime, cefetamet, ceftetrame, cefixime and tigemonam, demonstrate activity against $H$. pylori with $\mathrm{MIC}_{50}$ of $0.125,2,0.5,0.06$ and $0.25 \mu \mathrm{g} / \mathrm{mL}$, respectively. ${ }^{114}$ The antimycotic ketoconazole is active against forty $H$. pylori strains at $\mathrm{MIC}_{50}$ and $\mathrm{MIC}_{90}$ of 16 and $32 \mathrm{mg} / \mathrm{L}$ respectively. ${ }^{120}$ Mupirocin, a topical antimicrobial 
for the treatment of staphylococcal infection of the skin, show activity against 57 strains of $H$. pylori with $\mathrm{MIC}_{90}$ of 0.25 and $0.12 \mathrm{mg} / \mathrm{L}$ at $\mathrm{pH} 7.4$ and 5.4 respectively. ${ }^{121}$

Linezolid, an oxazolidone indicated for staphylococcal and streptococcal infections, has demonstrated activity against gram-negative bacteria. ${ }^{122}$ Linezolid in hibits 70 strains of $H$. pylori at the $\mathrm{MIC}_{50}$ of 8-64 $\mu \mathrm{g} / \mathrm{mL}$. Simethicone, a non-antibiotic and antifoaming agent used to treat meteorism, demonstrates anti- $H$. pylori activity with an MIC of $64-128 \mathrm{mg} / \mathrm{L} .{ }^{123} \mathrm{HSR}-903$, a quinolone compound, demonstrates anti- $H$. pylori activity with $\mathrm{MIC}_{50}$ of $0.20 \mu \mathrm{g} / \mathrm{mL}$ and $\mathrm{MIC}_{90}$ of $12.5 \mu \mathrm{g} / \mathrm{mL} .{ }^{124}$

\section{Research Goals}

In conclusion, new therapies for the treatment of $H$. pylori infections are needed, given the rise of resistance against current antibiotics. The development of narrow spectrum anti-H. pylori drugs should be prioritized to reduce side effects from disruption of the microbiome. Advances in whole genome sequencing have revealed many H. pylori targets for the discovery of selective anti-H. pylori compounds. The highthrough-put screen of commercial libraries is an attractive approach in the discovery of selective compounds against $H$. pylori. However, lead optimization campaign of compounds discovered from a phenotypic screen is more challenging and takes a long time compared to compounds that are developed through target-based drug discovery approach. The generation of mutants and genome sequencing techniques enable the study of mode of action of novel antimicrobials. To eradicate $H$. pylori in vivo, compounds must demonstrate the ability to reach the colonization site, in the mucus layer of the stomach. The goals of the research presented in this dissertation are to identify active compounds against $H$. pylori through the highthrough-put screening approach, to explore the hit compounds through the structure-activity relationship for improved potency and to optimize the in vitro and in vivo pharmacokinetic properties the resulting lead compounds for proof of concept studies in H. pylori infected mice. 


\title{
CHAPTER 2. THE DISCOVERY AND DEVELOPMENT OF THIENOPYRIMIDINES AS INHIBITORS OF HELICOBACTER PYLORI THROUGH INHIBITION OF THE RESPIRATORY COMPLEX I
}

\begin{abstract}
Introduction
Helicobacter pylori is a major causative agent of gastritis, peptic ulcers and ultimately gastric cancer. According to the Center for Disease Control, more than 500,000 cases of $H$. pylori infections are diagnosed annually in the United States. ${ }^{1}$ The triple therapy, consisting of a proton pump inhibitor and broad spectrum antibiotics clarithromycin and amoxicillin, is the recommended treatment against $H$. pylori infections. ${ }^{2}$ The rate of successful treatment with the triple therapy has dropped below $80 \%$ due to the rise of resistance..$^{3-6}$ In a recent study, $22 \%$ of patients tested in a clinical trial were not cured by the triple therapy. ${ }^{5}$ Additionally, the intensive use of the broadspectrum antibiotics for $\mathrm{H}$. pylori infections perturbs the gut microbiome, leading to side effects such as diarrhea and drug resistance in commensal pathogens. ${ }^{2}$ Given the rise of $H$. pylori resistance, new drugs and approaches are needed for the eradication of $H$. pylori infections.
\end{abstract}

A high-throughput screening protocol was developed that addressed the H. pylori challenge of growing $H$. pylori in a fastidious microaerophilic environment required for growth, to discover novel chemical matter active against $\mathrm{H}_{\text {. pylori. }}{ }^{7}$ Hits were then counter-screened against Staphylococcus aureus to remove promiscuous compounds, against gut commensal bacteria and mammalian cell lines to discover selective hits that can eradicate $H$. pylori while preserving the host microbiota. ${ }^{7}$ From this approach we have previously reported Hpil (Figure 2-1A), a selective anti-H. pylori compound that emerged as a hit, with excellent in vitro potency (H. pylori $\mathrm{IC}_{50}$ of $\left.0.24 \pm 0.04 \mu \mathrm{M}\right)$ compared to clarithromycin $(0.04 \pm 0.01 \mu \mathrm{M})$ and amoxicillin $(0.08 \pm 0.04 \mu \mathrm{M}){ }^{7}$ The screening approach was subsequently applied to a larger library of 219,197 compounds. ${ }^{8}$ 2,027 compounds emerged as positive hits and these compounds were clustered and subsequently prioritized based on their solubility and their chemical tractability. To eliminate generally toxic compounds, 511 were picked and counter-screened against $S$. aureus and gut commensals including Bifidocacterium longum ATCC BAA-999, Lactobacilus casei ATCC 334 and Lactobacillys reuteri ATXX 23272 and FaDu human cell line to eliminate promiscuous compounds. This screen identified two thienopyrimidine compounds, whose activity was confirmed by resynthesis and testing, $\mathbf{1}$ and 2 (Figure 2-1A), with the H. pylori $\mathrm{IC}_{50}$ values of $0.46 \mu \mathrm{g} / \mathrm{ml}$ and $0.59 \mu \mathrm{g} / \mathrm{ml}$ respectively as prospective series leads.

Herein, we report the structure-activity relationship developed from the optimization thienopyrimidines hits $\mathbf{1}$ and $\mathbf{2}$ against $H$. pylori, efforts that examine substitutions around the thienopyrmidine core and merge elements of the two hits. Leads are developed based on their activity and cytotoxicity profiles, and their efficacy in the in vivo and ex vivo H. pylori infection models in mice is examined. Through resistance generation, whole genome sequencing and resistance transfer, NuoD subunit of 


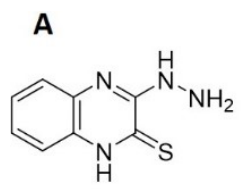

B

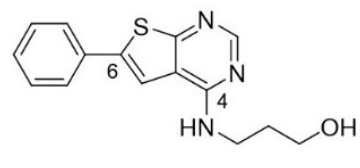

1
C

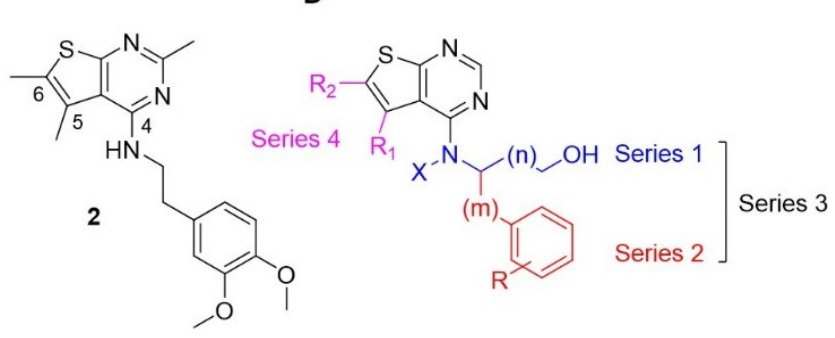

Figure 2-1. Structures of hit compounds from a highthrough-put screen against $H$. pylori and SAR development of the thienopyrimidines series.

(A) Structure of Hpi1, a selective anti-H.pylori compound that emerged as a positive hit from a high through-put screen against $H$. pylori. (B) and (C) Structures of

thienopyrimidines hits $\mathbf{1}$ and $\mathbf{2}$, that were identified using a similar screening approach with the H. pylori $\mathrm{IC}_{50}$ of $0.46 \mu \mathrm{g} / \mathrm{mL}$ and $0.59 \mu \mathrm{g} / \mathrm{mL}$ respectively. (D) The StructureActivity Relationship (SAR) of the thienopyrimidines against $H$. pylori is reported in four series, at the 4, 5 and 6 positions. 
Complex I was discovered as the putative target and validated by resistance transfer. A homology model of the putative $H$. pylori NuoD binding site was created and the binding mode of the leads examined. These studies produced tractable thienopyrimidine leads with a defined mechanism of action suitable for further optimization of their ADME and in vivo properties.

\section{Materials and Methods}

\section{Chemical Synthesis}

Starting materials were purchased from the major vendors such as Sigma-Aldrich and Enamine. Chemical reactions were monitored by the thin-layer chromatography (TLC) and the Waters ACQUITY-UPLC-MS-UV system. Microwave-assisted chemical reactions were carried out in the Biotage Initiator ${ }^{+}$. The reaction mixtures were purified using the Biotage Flash column chromatography system, with silica cartridges acquired from Biotage Inc. The solvents for chromatography were purchased from Sigma-Aldrich. NMR spectra were obtained using the Bruker $400 \mathrm{MHz}$ NMR spectrometer and the Bruker AVANCE $500 \mathrm{MHz}$ NMR spectrometer. NMR analysis was carried out using the MestReNova software. The chemical shifts and the coupling constants $(J)$ are reported in $\mathrm{ppm}$ and hertz $(\mathrm{Hz})$ respectively. The purity of compounds was determined to be $>95 \%$ by UPLC-MS-UV system and NMR. Optical rotation of the chiral compounds was determined using JASCO P-1010 Polarimeter.

\section{Synthesis of Thienopyrimidine Analogs}

Ethyl 2-amino-5-phenylthiophene-3-carboxylate (5). In a microwave tube, ethyl 3-cyanopropanoate $4(1.771 \mathrm{~mL}, 16.65 \mathrm{mmol})$ was added to a solution of 2phenylacetaldehyde $3(2 \mathrm{~g}, 16.65 \mathrm{mmol})$, sulfur $(0.587 \mathrm{~g}, 18.315 \mathrm{mmol})$ and morpholine $(1.579 \mathrm{~mL}, 18.315 \mathrm{mmol})$ in ethanol $(20 \mathrm{~mL})$. The resulting mixture was submitted to microwave heating for $30 \mathrm{~min}$ at $70^{\circ} \mathrm{C}$. After cooling, the mixture was filtered, and the filtrate was poured in water. The precipitate was collected, dried and purified by flash column chromatography (Isolera 1, Biotage, $25 \mathrm{~g}$ size, elution gradient 12-60\% ethyl acetate in hexanes) to afford $\mathbf{5}$ as a yellow solid.

Ethyl 2-amino-5-phenylthiophene-3-carboxylate (5). ${ }^{1} \mathrm{H}$ NMR (DMSO, 500 MHZ): $\delta$ 7.48-7.44 (m, 4H), $7.33(\mathrm{t}, J=7.7 \mathrm{~Hz}, 2 \mathrm{H}), 7.23(\mathrm{~s}, 1 \mathrm{H}), 7.19$ (t, $J=7.4 \mathrm{~Hz}$, $1 \mathrm{H}), 4.21$ (q, $J=7.1 \mathrm{~Hz}, 2 \mathrm{H}, 1.28(\mathrm{t}, J=7.1 \mathrm{~Hz}, 3 \mathrm{H})$; MS (ESI): $m / z 248.5\left(\mathrm{M}^{+}+\mathrm{H}\right)$.

6-phenylthieno[2,3-d]pyrimidin-4(3H)-one (6). Compound 5 (1.15 g, $4.65 \mathrm{mmol})$ was dissolved in excess formamide and the resulting mixture was heated for $16 \mathrm{~h}$ at $160^{\circ} \mathrm{C}$. The mixture was concentrated in vacuo and purified by flash column chromatography (Isolera 1, Biotage, $25 \mathrm{~g}$ size, elution gradient 12-60\% ethyl acetate in hexanes) to afford $\mathbf{6}$ as a red solid. 
6-phenylthieno[2,3-d]pyrimidin-4(3H)-one (6). ${ }^{1} \mathrm{H} \mathrm{NMR}\left(\mathrm{CDCl}_{3}, 500 \mathrm{MHZ}\right): \delta$ $11.05(\mathrm{~s}, 1 \mathrm{H}), 8.58(\mathrm{~s}, 1 \mathrm{H}), 7.65-7.60(\mathrm{~m}, 2 \mathrm{H}), 7.45(\mathrm{~s}, 1 \mathrm{H}), 7.43-7.38(\mathrm{~m}, 2 \mathrm{H}), 7.33-7.30$ (m, 1H); MS (ESI): $m / z 230.1\left(\mathrm{M}^{+}+\mathrm{H}\right)$.

4-chloro-6-phenylthieno[2,3-d]pyrimidine (7). Compound 6 (1 g, 4.38 mmol) and $\mathrm{N}, \mathrm{N}$-dimethylaniline $(0.043 \mathrm{~mL}, 0.336 \mathrm{mmol})$ were dissolved in phosphoryl trichloride $(5.10 \mathrm{~mL}, 54.8 \mathrm{mmol})$ and the mixture was heated for $16 \mathrm{~h}$ to $95^{\circ} \mathrm{C}$. The mixture was concentrated in vacuo and purified by flash column chromatography (Isolera 1, Biotage, $25 \mathrm{~g}$ size, elution gradient of $12-60 \%$ ethyl acetate in hexanes) to afford 7 as a yellow solid.

4-chloro-6-phenylthieno[2,3-d]pyrimidine (7). ${ }^{1} \mathrm{H} \mathrm{NMR}\left(\mathrm{CDCl}_{3}, 500 \mathrm{MHZ}\right): \delta$ 8.85 (s, 1H), 7.79-7.77 (m, 2H), 7.62 (s, 1H), 7.54-7.46 (m, 3H); MS (ESI): $m / z ~ 247.1$ $\left(\mathrm{M}^{+}+\mathrm{H}\right)$.

General procedure for the amination of 4-chloro-6-phenylthieno[2,3d]pyrimidine. In a microwave tube, compound 7 ( 1 equiv), amine (1.5 equiv) and triethylamine (3 equiv) were dissolved in ethanol and subjected to microwave heating for $1 \mathrm{~h}$ at $150^{\circ} \mathrm{C}$. The mixture was extracted with Ethyl Acetate/Water and the organic phase was washed with brine, dried $\left(\mathrm{Na}_{2} \mathrm{SO}_{4}\right)$ and concentrated in vacuo. The residue was purified by column chromatography (Isolera 1, Biotage, elution gradient of $20-85 \%$ ethyl acetate in hexanes) to afford the targeted compound.

\section{3-((6-phenylthieno[2,3-d]pyrimidin-4-yl)amino)propan-1-ol (1). ${ }^{1} \mathrm{H}$ NMR} $\left(\mathrm{CDCl}_{3}, 400 \mathrm{MHZ}\right): \delta 8.48(\mathrm{~s}, 1 \mathrm{H}), 7.69-7.67(\mathrm{~m}, 1 \mathrm{H}), 7.67-7.66(\mathrm{~m}, 1 \mathrm{H}), 7.48-7.43(\mathrm{~m}$, 2H), 7.41-7.37 (m, 1H), $7.33(\mathrm{~s}, 1 \mathrm{H}), 5.70(\mathrm{br}, 1 \mathrm{H}), 3.85(\mathrm{q}, J=6.5 \mathrm{~Hz}, 2 \mathrm{H}), 3.74(\mathrm{t}, J=$ $5.9 \mathrm{~Hz}, 2 \mathrm{H}), 1.93-1.87$ (m, 2H); MS (ESI): $m / z 286.1\left(\mathrm{M}^{+}+\mathrm{H}\right)$.

3-(methyl(6-phenylthieno[2,3-d]pyrimidin-4-yl)amino)propan-1-ol (8). ${ }^{1} \mathrm{H}$ NMR $\left(\mathrm{CDCl}_{3}, 400 \mathrm{MHZ}\right): \delta 8.41(\mathrm{~s}, 1 \mathrm{H}), 7.74(\mathrm{~s}, 1 \mathrm{H}), 7.70-7.64(\mathrm{~m}, 2 \mathrm{H}), 7.49-7.43(\mathrm{~m}, 2 \mathrm{H})$, 7.41-7.36 (m, 1H), 4.67 (br, 1H), $3.96(\mathrm{dd}, J=6.8,5.4 \mathrm{~Hz}, 2 \mathrm{H}), 3.61-3.60(\mathrm{~m}, 2 \mathrm{H}), 3.51$ (s, 3H), 1.96-1.90 (m, 2H); MS (ESI): $m / z 301.1\left(\mathrm{M}^{+}+\mathrm{H}\right)$.

2-((6-phenylthieno[2,3-d]pyrimidin-4-yl)amino)ethan-1-ol (9). ${ }^{1} \mathrm{H} \mathrm{NMR}\left(\mathrm{CDCl}_{3}\right.$, $400 \mathrm{MHz}) \delta 8.45(\mathrm{~d}, J=2.7 \mathrm{~Hz}, 1 \mathrm{H}), 7.72-7.60$ (m, 2H), 7.43 (ddt, $J=9.5,7.8,1.5 \mathrm{~Hz}$, 2H), 7.40-7.30 (m, 2H), $5.70(\mathrm{~s}, 1 \mathrm{H}), 3.93(\mathrm{dt}, J=5.0,3.3 \mathrm{~Hz}, 2 \mathrm{H}), 3.82$ (ddt, $J=7.2$, 5.4, $3.0 \mathrm{~Hz}, 2 \mathrm{H})$; MS (ESI): $m / z 273.1\left(\mathrm{M}^{+}+\mathrm{H}\right)$.

2-((6-phenylthieno[2,3-d]pyrimidin-4-yl)amino)propan-1-ol (10). ${ }^{1} \mathrm{H}$ NMR $\left(\mathrm{CDCl}_{3}, 400 \mathrm{MHZ}\right): \delta 8.38(\mathrm{~s}, 1 \mathrm{H}), 7.62-7.57(\mathrm{~m}, 2 \mathrm{H} 1.32(\mathrm{~d}, J=6.8 \mathrm{~Hz}, 3 \mathrm{H}), 7.37$ (ddd, $J=7.7,6.6,1.6 \mathrm{~Hz}, 2 \mathrm{H}), 7.31-7.25(\mathrm{~m}, 2 \mathrm{H}), 5.24(\mathrm{~d}, J=6.7 \mathrm{~Hz}, 1 \mathrm{H}), 4.42-4.40(\mathrm{~m}, 1 \mathrm{H})$, $3.81(\mathrm{dd}, J=10.9,3.1 \mathrm{~Hz}, 1 \mathrm{H}), 3.68-3.64(\mathrm{~m}, 1 \mathrm{H})$; MS (ESI): $m / z 286.9\left(\mathrm{M}^{+}+\mathrm{H}\right)$.

1-((6-phenylthieno[2,3-d]pyrimidin-4-yl)amino)propan-2-ol (11). ${ }^{1} \mathrm{H}$ NMR (DMSO, 400 MHZ): $\delta 8.33$ (s, 1H), $8.12(\mathrm{~s}, 1 \mathrm{H}), 7.99$ (t, $J=5.9 \mathrm{~Hz}, 1 \mathrm{H}), 7.69$ (d, $J=7.5$ 
$\mathrm{Hz}, 2 \mathrm{H}), 7.51(\mathrm{t}, J=6.8 \mathrm{~Hz}, 2 \mathrm{H}), 7.42-7.38(\mathrm{~m}, J=7.5 \mathrm{~Hz}, 1 \mathrm{H}), 4.86(\mathrm{~d}, J=4.8 \mathrm{~Hz}, 1 \mathrm{H})$, 3.94-3.89 (m, 1H), 3.54-3.38 (m, 2H), 1.13 (d, $J=6.2 \mathrm{~Hz}, 3 \mathrm{H})$; MS (ESI): $m / z 268.7$ $\left(\mathrm{M}^{+}+\mathrm{H}\right)$.

(S)-2-((6-phenylthieno[2,3-d]pyrimidin-4-yl)amino)propan-1-ol (12). ${ }^{1} \mathrm{H}$ NMR $\left(\mathrm{CDCl}_{3}, 400 \mathrm{MHZ}\right): \delta 8.47(\mathrm{~s}, 1 \mathrm{H}), 7.71-7.68(\mathrm{~m}, 2 \mathrm{H}), 7.46(\mathrm{t}, J=7.5 \mathrm{~Hz}, 2 \mathrm{H}), 7.41-7.39$ $(\mathrm{m}, 1 \mathrm{H}), 7.35(\mathrm{~s}, 1 \mathrm{H}), 5.31(\mathrm{~s}, 1 \mathrm{H}), 4.51-4.49(\mathrm{~m}, 1 \mathrm{H}), 3.91(\mathrm{dd}, J=11.1,3.02 \mathrm{~Hz}, 1 \mathrm{H})$, 3.75 (dd, $J=11.1,6.2 \mathrm{~Hz}, 1 \mathrm{H}), 1.41(\mathrm{~d}, J=6.9 \mathrm{~Hz}, 3 \mathrm{H})$; MS (ESI): $m / z 286.9\left(\mathrm{M}^{+}+\mathrm{H}\right)$.

(R)-2-((6-phenylthieno[2,3-d]pyrimidin-4-yl)amino)propan-1-ol (13). ${ }^{1} \mathrm{H}$ NMR $\left(\mathrm{CDCl}_{3}, 400 \mathrm{MHZ}\right): \delta 8.47(\mathrm{~s}, 1 \mathrm{H}), 7.70-7.68(\mathrm{~m}, 2 \mathrm{H}), 7.46(\mathrm{t}, J=8.2 \mathrm{~Hz}, 2 \mathrm{H}), 7.41-7.35$ $(\mathrm{m}, 2 \mathrm{H}), 5.33(\mathrm{~s}, 1 \mathrm{H}), 4.52-4.48(\mathrm{~m}, 1 \mathrm{H}), 3.93-3.88(\mathrm{~m}, 1 \mathrm{H}), 3.78-3.73(\mathrm{~m}, 1 \mathrm{H}), 1.41(\mathrm{~d}, J$ $=6.4 \mathrm{~Hz}, 3 \mathrm{H})$; MS (ESI): $m / z 286.9\left(\mathrm{M}^{+}+\mathrm{H}\right)$.

N-(1-methoxypropan-2-yl)-6-phenylthieno[2,3-d]pyrimidin-4-amine (14). ${ }^{1} \mathrm{H}$ NMR (DMSO, 400 MHZ): $\delta 8.34(\mathrm{~s}, 1 \mathrm{H}), 8.12(\mathrm{~s}, 1 \mathrm{H}), 7.74-7.68(\mathrm{~m}, 3 \mathrm{H}), 7.51(\mathrm{t}, 2 \mathrm{H}, J$ $=7.4 \mathrm{~Hz}), 7.43-7.38(\mathrm{~m}, 1 \mathrm{H}), 4.60-4.53(\mathrm{~m}, 1 \mathrm{H}), 3.52-3.48(\mathrm{~m}, 1 \mathrm{H}), 3.40-3.37(\mathrm{~m}, 1 \mathrm{H})$, $3.30(\mathrm{~s}, 3 \mathrm{H}), 1.24(\mathrm{~d}, J=6.7 \mathrm{~Hz}, 3 \mathrm{H})$; MS (ESI): $m / z 300.8\left(\mathrm{M}^{+}+\mathrm{H}\right)$.

4-((6-phenylthieno[2,3-d]pyrimidin-4-yl)amino)butan-1-ol (15). ${ }^{1} \mathrm{H}$ NMR $\left(\mathrm{CDCl}_{3}, 400 \mathrm{MHZ}\right): \delta 8.47(\mathrm{~s}, 1 \mathrm{H}), 7.67-7.64(\mathrm{~m}, 2 \mathrm{H}), 7.45-7.25(\mathrm{~m}, 4 \mathrm{H}), 5.67(\mathrm{~s}, 1 \mathrm{H})$, 3.79 (s, 2H), 3.69 (s, 2H), 2.16 (s, 1H), 1.87-1.84 (m, 2H), 1.75-1.73 (m, 2H); MS (ESI): $\mathrm{m} / \mathrm{z} 301.3\left(\mathrm{M}^{+}+\mathrm{H}\right)$.

2-((6-phenylthieno[2,3-d]pyrimidin-4-yl)amino)butan-1-ol (16). ${ }^{1} \mathrm{H}$ NMR $\left(\mathrm{CDCl}_{3}, 400 \mathrm{MHZ}\right): \delta 8.45(\mathrm{~s}, 1 \mathrm{H}), 7.70-7.67(\mathrm{~m}, 2 \mathrm{H}), 7.48-7.43(\mathrm{~m}, 2 \mathrm{H}), 7.39(\mathrm{t}, J=$ $7.48 \mathrm{~Hz}, 1 \mathrm{H}), 7.35(\mathrm{~s}, 1 \mathrm{H}), 5.34(\mathrm{~d}, J=7.5 \mathrm{~Hz}, 1 \mathrm{H}), 4.33-4.26(\mathrm{~m}, 1 \mathrm{H}), 3.93(\mathrm{dd}, J=$ 11.1, $3 \mathrm{~Hz}, 1 \mathrm{H}), 3.80(\mathrm{dd}, J=11.1,5.8 \mathrm{~Hz}, 1 \mathrm{H}), 1.88-1.70(\mathrm{~m}, 2 \mathrm{H}), 1.09$ (t, $J=7.2 \mathrm{~Hz}$, $3 \mathrm{H})$; MS (ESI): $m / z 300.9\left(\mathrm{M}^{+}+\mathrm{H}\right)$.

1-((6-phenylthieno[2,3-d]pyrimidin-4-yl)amino)butan-2-ol (17). ${ }^{1} \mathrm{H}$ NMR (DMSO, 400 MHZ): $\delta 8.33(\mathrm{~s}, 1 \mathrm{H}), 8.12(\mathrm{~s}, 1 \mathrm{H}), 7.96(\mathrm{t}, J=5.73 \mathrm{~Hz}, 1 \mathrm{H}), 7.70-7.67$ (m, 2H), $7.51(\mathrm{dd}, J=8.41,7.01 \mathrm{~Hz}, 2 \mathrm{H}), 7.42-7.38(\mathrm{~m}, 1 \mathrm{H}), 4.83(\mathrm{~d}, J=5.24 \mathrm{~Hz}, 1 \mathrm{H}), 3.70-$ $3.62(\mathrm{~m}, 1 \mathrm{H}), 3.61-3.55(\mathrm{~m}, 1 \mathrm{H}), 3.44-3.37(\mathrm{~m}, 1 \mathrm{H}), 1.58-1.48(\mathrm{~m}, 1 \mathrm{H}), 1.42-1.31(\mathrm{~m}$, $1 \mathrm{H}), 0.93$ (t, $J=7.4 \mathrm{~Hz}, 3 \mathrm{H})$; MS (ESI): $m / z 300.9\left(\mathrm{M}^{+}+\mathrm{H}\right)$.

3-methyl-2-((6-phenylthieno[2,3-d]pyrimidin-4-yl)amino)butan-1-ol (18). ${ }^{1} \mathrm{H}$ NMR ( $\left.\mathrm{CDCl}_{3}, 400 \mathrm{MHZ}\right): \delta 8.35(\mathrm{~s}, 1 \mathrm{H}), 7.62-7.59(\mathrm{~m}, 2 \mathrm{H}), 7.37(\mathrm{t}, J=7.2 \mathrm{~Hz}, 2 \mathrm{H})$, 7.32-7.25 (m, 2H), $5.30(\mathrm{~d}, J=7.7 \mathrm{~Hz}, 1 \mathrm{H}), 4.11-4.05(\mathrm{~m}, 1 \mathrm{H}), 3.87-3.75(\mathrm{~m}, 2 \mathrm{H}), 3.48$ (m, 1H), 2.10-2.01 (m, 1H), 1.20-0.98 (m, 6H); MS (ESI): $m / z 315.01\left(\mathrm{M}^{+}+\mathrm{H}\right)$.

N-(3,4-dimethoxyphenethyl)-6-phenylthieno[2,3-d]pyrimidin-4-amine (19). ${ }^{1} \mathrm{H}$ NMR ( $\left.\mathrm{CDCl}_{3}, 400 \mathrm{MHZ}\right): \delta 8.54(\mathrm{~s}, 1 \mathrm{H}), 7.65(\mathrm{~d}, J=7 \mathrm{~Hz}, 2 \mathrm{H}), 7.45$ (t, J= $7.04 \mathrm{~Hz}$, 2H), 7.40-7.36 (m, 1H), 7.20 (s, 1H), 6.88-6.78 (m, 3H), 5.28 (br, 1H), 3.95-3.90 (m, 5H), 3.87 (s, 3H), 2.99 (t, $J=7.04 \mathrm{~Hz}, 2 \mathrm{H})$; MS (ESI): $m / z 392.01\left(\mathrm{M}^{+}+\mathrm{H}\right)$. 
N-(3,4-dimethylphenethyl)-6-phenylthieno[2,3-d]pyrimidin-4-amine (20). ${ }^{1} \mathrm{H}$ NMR (DMSO, $400 \mathrm{MHZ}): \delta 8.37(\mathrm{~s}, 1 \mathrm{H}), 8.07$ (t, $J=5.62 \mathrm{~Hz}, 1 \mathrm{H}), 8.00(\mathrm{~s}, 1 \mathrm{H}), 7.67$ (d, $J=7.52 \mathrm{~Hz}, 2 \mathrm{H}), 7.51(\mathrm{t}, J=8.08 \mathrm{~Hz}, 2 \mathrm{H}), 7.40(\mathrm{t}, J=7.12 \mathrm{~Hz}, 1 \mathrm{H}), 7.06-7.05(\mathrm{~m}, 2 \mathrm{H})$, 6.99-6.97 (m, 1H), 3.73-3.68 (m, 2H), 2.87 (t, $J=7.49$ Hz, 2H), 2.19 (s, 3H), 2.17 (s, $3 \mathrm{H})$; MS (ESI): $m / z 361.21\left(\mathrm{M}^{+}+\mathrm{H}\right)$.

6-phenyl-N-(2-(pyridin-4-yl)ethyl)thieno[2,3-d]pyrimidin-4-amine (21). ${ }^{1} \mathrm{H}$ NMR $\left(\mathrm{CDCl}_{3}, 400 \mathrm{MHZ}\right): \delta$ 8.56-8.47 (m, 3H), 7.65-7.63 (m, 2H), 7.44-7.41 (m, 2H), 7.38-7.36 (m, 1H), 7.21-7.18 (m, 3H), $5.26(\mathrm{~s}, 1 \mathrm{H}), 3.98-3.93(\mathrm{~m}, 2 \mathrm{H}), 3.05(\mathrm{t}, 2 \mathrm{H}, J=$ $7.52 \mathrm{~Hz})$; MS (ESI): $m / z 333.1\left(\mathrm{M}^{+}+\mathrm{H}\right)$.

6-phenyl-N-(2-(pyridin-3-yl)ethyl)thieno[2,3-d]pyrimidin-4-amine (22). ${ }^{1} \mathrm{H}$ NMR ( $\left.\mathrm{CDCl}_{3}, 400 \mathrm{MHZ}\right): \delta 8.56-8.53(\mathrm{~m}, 3 \mathrm{H}), 7.68-7.65(\mathrm{~m}, J=6.88 \mathrm{~Hz}, 2 \mathrm{H}), 7.61(\mathrm{~d}$, $J=7.91 \mathrm{~Hz}, 1 \mathrm{H}), 7.45(\mathrm{dd}, J=8.45,6.60 \mathrm{~Hz}, 2 \mathrm{H}), 7.40-7.37(\mathrm{~m}, 1 \mathrm{H}), 7.24(\mathrm{~s}, 1 \mathrm{H}), 5.26$ $(\mathrm{s}, 1 \mathrm{H}) 3.96(\mathrm{q}, J=6.73,2 \mathrm{H}), 3.08(\mathrm{t}, J=6.96 \mathrm{~Hz}, 2 \mathrm{H})$; MS (ESI): $m / z 333.1\left(\mathrm{M}^{+}+\mathrm{H}\right)$.

N-(2,3-dimethoxybenzyl)-6-phenylthieno[2,3-d]pyrimidin-4-amine (23). ${ }^{1} \mathrm{H}$ NMR $\left(\mathrm{CDCl}_{3}, 400 \mathrm{MHz}\right) \delta 8.54$ (s, 1H), 7.69-7.63 (m, 2H), 7.49-7.41 (m, 2H), 7.40-7.33 $(\mathrm{m}, 1 \mathrm{H}), 7.30(\mathrm{~d}, J=1.2 \mathrm{~Hz}, 1 \mathrm{H}), 7.11-7.00(\mathrm{~m}, 2 \mathrm{H}), 6.94(\mathrm{dd}, J=7.8,1.8 \mathrm{~Hz}, 1 \mathrm{H}), 5.73$ (s, 1H), 4.89 (d, $J=5.6 \mathrm{~Hz}, 2 \mathrm{H}), 3.97$ (d, $J=0.9 \mathrm{~Hz}, 3 \mathrm{H}), 3.92$ (d, $J=1.1 \mathrm{~Hz}, 3 \mathrm{H})$; MS (ESI): $m / z 379.02\left(\mathrm{M}^{+}+\mathrm{H}\right)$.

3-(4-methoxyphenyl)-2-((6-phenylthieno[2,3-d]pyrimidin-4-yl)amino)propan-1ol (24). ${ }^{1} \mathrm{H}$ NMR $\left(\mathrm{CDCl}_{3}, 500 \mathrm{MHZ}\right): \delta 8.38$ (s, 1H), 7.55 (d, $\left.J=7.65 \mathrm{~Hz}, 2 \mathrm{H}\right), 7.36$ (t, $J$ $=7.54 \mathrm{~Hz}, 2 \mathrm{H}), 7.30(\mathrm{t}, J=7.7 \mathrm{~Hz}, 1 \mathrm{H}), 7.14-7.11(\mathrm{~m}, 3 \mathrm{H}), 6.80(\mathrm{~d}, J=8.2 \mathrm{~Hz}, 2 \mathrm{H}), 5.39$ $(\mathrm{d}, J=6.25 \mathrm{~Hz}, 1 \mathrm{H}), 4.45-4.39(\mathrm{~m}, 1 \mathrm{H}), 3.82(\mathrm{~d}, J=10.8 \mathrm{~Hz}, 1 \mathrm{H}), 3.72-3.66(\mathrm{~m}, 4 \mathrm{H})$, 2.97-2.80 (m, 2H); MS (ESI): $m / z 391.9\left(\mathrm{M}^{+}+\mathrm{H}\right)$.

2-(4-methoxyphenyl)-2-((6-phenylthieno[2,3-d]pyrimidin-4-yl)amino)ethan-1-ol (25). ${ }^{1} \mathrm{H} \mathrm{NMR}\left(\mathrm{CDCl}_{3}, 400 \mathrm{MHZ}\right): \delta 8.38(\mathrm{~s}, 1 \mathrm{H}), 7.59(\mathrm{dd}, J=8.19,1.45 \mathrm{~Hz}, 2 \mathrm{H}), 7.36$ $(\mathrm{td}, J=7.63,6.91,1.15 \mathrm{~Hz}, 2 \mathrm{H}), 7.32-7.27(\mathrm{~m}, 4 \mathrm{H}), 6.86(\mathrm{~d}, J=8.48 \mathrm{~Hz}, 2 \mathrm{H}), 5.65(\mathrm{~d}, J$ $=6.22 \mathrm{~Hz}, 1 \mathrm{H}), 5.35-5.31(\mathrm{~m}, 1 \mathrm{H}), 4.01-3.98(\mathrm{~m}, 2 \mathrm{H}), 3.75(\mathrm{~s}, 3 \mathrm{H}), 3.57(\mathrm{~s}, 1 \mathrm{H})$; MS (ESI): $m / z 378.1\left(\mathrm{M}^{+}+\mathrm{H}\right)$.

(R)-2-(4-methoxyphenyl)-2-((6-phenylthieno[2,3-d]pyrimidin-4-yl)amino)ethan1-ol (26). ${ }^{1} \mathrm{H}$ NMR (DMSO, $\left.400 \mathrm{MHZ}\right): \delta 8.28$ (s, $\left.1 \mathrm{H}\right), 8.24(\mathrm{~s}, 1 \mathrm{H}), 8.13(\mathrm{~d}, J=8.2 \mathrm{~Hz}$, $1 \mathrm{H}), 7.73-7.70(\mathrm{~m}, 2 \mathrm{H}), 7.54-7.50(\mathrm{~m}, J=8.12 \mathrm{~Hz}, 2 \mathrm{H}), 7.43-7.39(\mathrm{~m}, 1 \mathrm{H}), 7.37-7.34(\mathrm{~m}$, 2H), $6.89(\mathrm{~d}, J=8.6 \mathrm{~Hz}, 2 \mathrm{H}), 5.43-5.38(\mathrm{~m}, 1 \mathrm{H}), 4.99(\mathrm{t}, J=6.16 \mathrm{~Hz}, 1 \mathrm{H}), 3.79-3.69$ (m, $5 \mathrm{H})$; MS (ESI): $m / z 378.1\left(\mathrm{M}^{+}+\mathrm{H}\right)$.

(S)-2-(4-methoxyphenyl)-2-((6-phenylthieno[2,3-d]pyrimidin-4-yl)amino)ethan1-ol (27). ${ }^{1} \mathrm{H}$ NMR (DMSO, $\left.400 \mathrm{MHZ}\right): \delta 8.28$ (s, 1H), 8.24 (s, 1H), 8.14 (d, J=7.76 Hz, $1 \mathrm{H}), 7.72(\mathrm{~d}, J=8.2 \mathrm{~Hz}, 2 \mathrm{H}), 7.52$ (t, $J=7.72 \mathrm{~Hz}, 2 \mathrm{H}), 7.43-7.35(\mathrm{~m}, 3 \mathrm{H}), 6.89$ (d, $J=9$ $\mathrm{Hz}, 2 \mathrm{H}), 5.41$ (q, $J=7.88 \mathrm{~Hz}, 1 \mathrm{H}), 4.99$ (t, $J=6.04 \mathrm{~Hz}, 1 \mathrm{H}), 3.79-3.69$ (m, 5H); MS (ESI): $m / z 378.1\left(\mathrm{M}^{+}+\mathrm{H}\right)$. 
(R)-2-phenyl-2-((6-phenylthieno[2,3-d]pyrimidin-4-yl)amino)ethan-1-ol (28). ${ }^{1} \mathrm{H}$ NMR (CDCl $3,400 \mathrm{MHZ}): \delta 8.46(\mathrm{~s}, 1 \mathrm{H}), 7.69$ (d, $J=6.88 \mathrm{~Hz}, 2 \mathrm{H}), 7.47-7.34(\mathrm{~m}, 9 \mathrm{H})$, $5.85(\mathrm{~s}, 1 \mathrm{H}), 5.50-5.47$ (m, 1H), 4.17-4.09 (m, 2H); MS (ESI): $m / z 348.9\left(\mathrm{M}^{+}+\mathrm{H}\right)$.

(R)-2-(2-fluoro-4-methoxyphenyl)-2-((6-phenylthieno[2,3-d]pyrimidin-4yl)amino)ethan-1-ol (29). ${ }^{1} \mathrm{H}$ NMR ( $\left.\mathrm{CDCl}_{3}, 400 \mathrm{MHZ}\right): \delta 8.45$ (s, 1H), 7.67 (d, $J=6.16$ $\mathrm{Hz}, 2 \mathrm{H}), 7.45-7.29$ (m, 5H), $6.70(\mathrm{~s}, 2 \mathrm{H}), 6.02$ (s, 1H), $5.70(\mathrm{~s}, 1 \mathrm{H}), 4.14-4.05(\mathrm{~m}, 2 \mathrm{H})$, $3.81(\mathrm{~s}, 3 \mathrm{H})$; MS (ESI): $m / z 396.01\left(\mathrm{M}^{+}+\mathrm{H}\right)$.

(R)-2-(2-fluoro-4-methoxyphenyl)-2-((6-phenylthieno[2,3-d]pyrimidin-4yl)amino)ethan-1-ol (30). ${ }^{1} \mathrm{H} \mathrm{NMR}\left(\mathrm{CDCl}_{3}, 400 \mathrm{MHZ}\right): \delta 8.42(\mathrm{~s}, 1 \mathrm{H}), 7.67-7.65(\mathrm{~m}$, 2H), $7.54(\mathrm{~s}, 1 \mathrm{H}), 7.45-7.35(\mathrm{~m}, 3 \mathrm{H}), 7.22-7.16(\mathrm{~m}, 2 \mathrm{H}), 6.98(\mathrm{t}, J=8.6 \mathrm{~Hz}, 1 \mathrm{H}), 6.31(\mathrm{~s}$, 1H), 5.45-5.41 (m, 1H), 4.14-4.06 (m, 2H), 3.90 (s, 3H); MS (ESI): m/z $397.3\left(\mathrm{M}^{+}+\mathrm{H}\right)$.

Compounds 37-40 were synthesized following the General procedure for the amination of 4-chloro-6-phenylthieno[2,3-d]pyrimidine.

(R)-2-((5,6-dimethylthieno[2,3-d]pyrimidin-4-yl)amino)-2-phenylethan-1-ol (37). ${ }^{1} \mathrm{H}$ NMR $\left(\mathrm{CDCl}_{3}, 400 \mathrm{MHZ}\right): \delta 8.35(\mathrm{~s}, 1 \mathrm{H}), 7.44-7.28(\mathrm{~m}, 5 \mathrm{H}), 6.28(\mathrm{~s}, 1 \mathrm{H}), 5.47-$ $5.43(\mathrm{~m}, 1 \mathrm{H}), 4.07(\mathrm{~d}, J=4.58 \mathrm{~Hz}, 2 \mathrm{H}), 2.51$ (s, 3H), 2.46 (s, 3H); MS (ESI): $m / z 301.00$ $\left(\mathrm{M}^{+}+\mathrm{H}\right)$.

(R)-2-((5-methyl-6-phenylthieno[2,3-d]pyrimidin-4-yl)amino)-2-phenylethan-1ol (38). ${ }^{1} \mathrm{H} \mathrm{NMR}\left(\mathrm{CDCl}_{3}, 400 \mathrm{MHZ}\right)$ : $\delta 8.48$ (s, 1H), 7.48-7.36 (m, 9H), 7.11-7.07 (m, $1 \mathrm{H}), 5.89$ (d, $J=6.84 \mathrm{~Hz}, 1 \mathrm{H}), 5.49$ (q, $J=5.44 \mathrm{~Hz}, 1 \mathrm{H}), 4.17-4.09$ (m, 2H), 1.28 (s, 3H); MS (ESI): $m / z 361.9\left(\mathrm{M}^{+}+\mathrm{H}\right)$.

(R)-2-((6-methyl-5-phenylthieno[2,3-d]pyrimidin-4-yl)amino)-2-phenylethan-1ol (39). ${ }^{1} \mathrm{H}$ NMR $\left(\mathrm{CDCl}_{3}, 400 \mathrm{MHZ}\right): \delta 8.42$ (s, 1H), 7.57 (t, $\left.J=7.63 \mathrm{~Hz}, 1 \mathrm{H}\right), 7.45-7.41$ $(\mathrm{m}, 2 \mathrm{H}), 7.27-7.21(\mathrm{~m}, 5 \mathrm{H}), 6.90-6.88(\mathrm{~m}, 2 \mathrm{H}), 5.33(\mathrm{~d}, J=5.6 \mathrm{~Hz}, 1 \mathrm{H}), 5.20-5.16(\mathrm{~m}$, $1 \mathrm{H}), 3.78-3.69$ (m, 2H), 2.33 (s, 3H); MS (ESI): $m / z 361.9\left(\mathrm{M}^{+}+\mathrm{H}\right)$.

(R)-2-phenyl-2-((5-phenylthieno[2,3-d]pyrimidin-4-yl)amino)ethan-1-ol (40). ${ }^{1} \mathrm{H}$ NMR ( $\left.\mathrm{CDCl}_{3}, 400 \mathrm{MHZ}\right): \delta 8.45(\mathrm{~s}, 1 \mathrm{H}), 7.69-7.67(\mathrm{~m}, 2 \mathrm{H}), 7.47-7.35(\mathrm{~m}, 9 \mathrm{H}), 5.96-$ $5.94(\mathrm{~m}, 1 \mathrm{H}), 5.51-5.47(\mathrm{~m}, 1 \mathrm{H}), 4.17-4.10(\mathrm{~m}, 2 \mathrm{H})$; MS (ESI): $\mathrm{m} / z 348.1\left(\mathrm{M}^{+}+\mathrm{H}\right)$.

\section{Synthesis of Amine Analog 31}

tert-butyl (R)-(1-(3-fluoro-4-methoxyphenyl)-2-hydroxyethyl)carbamate (33). Di-tert-butyl dicarbonate $(0.290 \mathrm{~g}, 1.329 \mathrm{mmol})$ was added to a solution of (R)-1-(3fluoro-4-methoxyphenyl)-2-hydroxyethanaminium chloride 32 (0.267 g, $1.208 \mathrm{mmol})$ and triethylamine $(0.506 \mathrm{~mL}, 3.63 \mathrm{mmol})$ in DCM $(4 \mathrm{~mL})$. The reaction mixture was stirred for $2 \mathrm{~h}$ at room temperature. The mixture was concentrated and purified by flash column chromatography (Isolera 1, Biotage, $10 \mathrm{~g}$ size, elution gradient $12-80 \%$ ethyl acetate in hexanes). Pure fractions were evaporated to afford $\mathbf{3 3}$ as a white solid. 
tert-butyl (R)-(1-(3-fluoro-4-methoxyphenyl)-2-hydroxyethyl)carbamate (33). ${ }^{1} \mathrm{H}$ NMR $\left(\mathrm{CDCl}_{3}, 500 \mathrm{MHZ}\right): \delta 7.08-7.03(\mathrm{~m}, 2 \mathrm{H}), 6.96(\mathrm{t}, J=8.33 \mathrm{~Hz}, 1 \mathrm{H}), 5.21(\mathrm{~d}, J=$ $6.55 \mathrm{~Hz}, 1 \mathrm{H}), 4.73(\mathrm{~s}, 1 \mathrm{H}), 3.90-3.81(\mathrm{~m}, 5 \mathrm{H}), 2.11(\mathrm{~s}, 1 \mathrm{H}), 1.46(\mathrm{~s}, 9 \mathrm{H}) ; \mathrm{MS}(\mathrm{ESI}): \mathrm{m} / z$ $284.3\left(\mathrm{M}^{+}+\mathrm{H}\right)$.

tert-butyl (R)-(2-(1,3-dioxoisoindolin-2-yl)-1-(3-fluoro-4methoxyphenyl)ethyl)carbamate (34). ${ }^{\mathbf{1 4 4}}$ DIAD (0.282 mL, $\left.1.458 \mathrm{mmol}\right)$ was added dropwise at room temperature, to a solution of 33 ( $0.320 \mathrm{~g}, 1.122 \mathrm{mmol})$, phthalimide $(0.215 \mathrm{~g}, 1.458 \mathrm{mmol})$ and $\mathrm{Ph}_{3} \mathrm{P}(0.382 \mathrm{~g}, 1.458 \mathrm{mmol})$ in THF $(3.8 \mathrm{~mL})$. The resulting mixture was stirred for $16 \mathrm{~h}$ at room temperature. The mixture was extracted with Ethyl Acetate/Water and the organic phase was washed with brine, dried $\left(\mathrm{Na}_{2} \mathrm{SO}_{4}\right)$ and evaporated in vacuo. The residue was purified by flash column chromatography (Isolera 1, Biotage, $10 \mathrm{~g}$ size, elution gradient $12-60 \%$ ethyl acetate in hexanes), to afford $\mathbf{3 4}$ as a yellow solid.

tert-butyl (R)-(2-(1,3-dioxoisoindolin-2-yl)-1-(3-fluoro-4methoxyphenyl)ethyl)carbamate (34). ${ }^{1} \mathrm{H} \mathrm{NMR}\left(\mathrm{CDCl}_{3}, 500 \mathrm{MHZ}\right): \delta 7.80(\mathrm{~m}, 2 \mathrm{H}), 7.66$ $(\mathrm{m}, 2 \mathrm{H}), 7.04(\mathrm{~m}, 2 \mathrm{H}), 6.87(\mathrm{~m}, 1 \mathrm{H}), 5.28(\mathrm{~d}, J=8.1 \mathrm{~Hz}, 1 \mathrm{H}), 4.94-4.92(\mathrm{~m}, 1 \mathrm{H}), 3.89-$ $3.81(\mathrm{~m}, 5 \mathrm{H}), 1.20(\mathrm{~s}, 9 \mathrm{H})$; MS (ESI): $m / z 415.7\left(\mathrm{M}^{+}+\mathrm{H}\right)$.

(R)-2-(2-amino-2-(3-fluoro-4-methoxyphenyl)ethyl)isoindoline-1,3-dione (35) and (R)-2-(2-(3-fluoro-4-methoxyphenyl)-2-((6-phenylthieno[2,3-d]pyrimidin-4yl)amino)ethyl)isoindoline-1,3-dione (36). $\mathrm{HCl}$ (4 M in 1,4-dioxane, $0.9 \mathrm{~mL}, 3.6 \mathrm{mmol}$ ) was added to a solution of $34(0.372 \mathrm{~g}, 0.897 \mathrm{mmol})$ in a mixture of DCM $(2.5 \mathrm{~mL})$ and methanol $(1 \mathrm{~mL})$, and the resulting mixture was stirred for $16 \mathrm{~h}$ at room temperature. The mixture was subjected to acid-base extraction with Ethyl Acetate/Water and the organic phase was washed with brine, dried $\left(\mathrm{Na}_{2} \mathrm{SO}_{4}\right)$ and concentrated in vacuo. The resulting residue 35 (yellow oil) was used in the next step without further purification. 36 was synthesized following the general procedure for amination of 4-chloro-6phenylthieno[2,3- $d]$ pyrimidine.

(R)-2-(2-amino-2-(3-fluoro-4-methoxyphenyl)ethyl)isoindoline-1,3-dione (35). ${ }^{1} \mathrm{H}$ NMR (MeOD, $\left.500 \mathrm{MHZ}\right): \delta$ 7.88-7.78 (m, 4H), 7.20 (dd, $\left.J=12.4,2.20 \mathrm{~Hz}, 1 \mathrm{H}\right), 7.08$ $(\mathrm{dd}, J=8.65,2.14 \mathrm{~Hz}, 1 \mathrm{H}), 7.00(\mathrm{t}, J=8.49 \mathrm{~Hz}, 1 \mathrm{H}), 4.25$ (t, $J=7.27 \mathrm{~Hz}, 1 \mathrm{H}), 3.88-3.84$ (m, 5H); MS (ESI): $m / z 315.5\left(\mathrm{M}^{+}+\mathrm{H}\right)$.

(R)-2-(2-(3-fluoro-4-methoxyphenyl)-2-((6-phenylthieno[2,3-d]pyrimidin-4yl)amino)ethyl)isoindoline-1,3-dione (36). ${ }^{1} \mathrm{H} \mathrm{NMR}\left(\mathrm{CDCl}_{3}, 500 \mathrm{MHZ}\right): \delta 8.31(\mathrm{~s}, 1 \mathrm{H})$, 7.88-7.85 (m, 2H), 7.75-7.73 (m, 4H), $7.54(\mathrm{~s}, 1 \mathrm{H}), 7.49(\mathrm{t}, J=7.55,1 \mathrm{H}), 7.42(\mathrm{t}, J=$ $7.35 \mathrm{~Hz}, 1 \mathrm{H}), 7.25-7.22(\mathrm{~m}, 2 \mathrm{H}), 6.99-6.95(\mathrm{~m}, 1 \mathrm{H}), 6.91(\mathrm{~s}, 1 \mathrm{H}), 5.66-5.62(\mathrm{~m}, 1 \mathrm{H})$, 4.24-4.12 (m, 2H), 3.89 (s, 3H); MS (ESI): $m / z ~ 525.5\left(\mathrm{M}^{+}+\mathrm{H}\right)$.

(R)-1-(3-fluoro-4-methoxyphenyl)- $N^{1}$-(6-phenylthieno[2,3-d]pyrimidin-4yl)ethane-1,2-diamine (31). Compound 36 ( $0.203 \mathrm{~g}, 0.393 \mathrm{mmol}$ ) was dissolved in methanol and a $80 \%$ hydrazine hydrate solution $(0.073 \mathrm{~mL}, 1.2 \mathrm{mmol})$ was added dropwise to the solution at $0^{\circ} \mathrm{C}$. The reaction mixture was stirred for $16 \mathrm{~h}$ at room 
temperature. The mixture was extracted with Ethyl Acetate/Water and the organic phase was washed with brine, dried $\left(\mathrm{Na}_{2} \mathrm{SO}_{4}\right)$ and evaporated in vacuo. The residue was purified by flash column chromatography (Isolera 1, Biotage, $10 \mathrm{~g}$ size, elution gradient $2-20 \%$ methanol in ethyl acetate) to afford 31 as a yellow solid.

(R)-1-(3-fluoro-4-methoxyphenyl)-N $N^{1}-(6-p h e n y l t h i e n o[2,3-d] p y r i m i d i n-4-$ yl)ethane-1,2-diamine (31). ${ }^{1} \mathrm{H}$ NMR (DMSO, $\left.500 \mathrm{MHZ}\right): \delta 8.27(\mathrm{~s}, 1 \mathrm{H}), 8.15(\mathrm{~s}, 1 \mathrm{H})$, $7.75(\mathrm{~d}, 2 \mathrm{H}, J=7.6 \mathrm{~Hz}), 7.54(\mathrm{t}, 2 \mathrm{H}, J=7.6 \mathrm{~Hz}), 7.44$ (t, $1 \mathrm{H}, J=7.6 \mathrm{~Hz}), 7.26(\mathrm{dd}, J=$ $12.5,2 \mathrm{~Hz}, 1 \mathrm{H}), 7.20(\mathrm{~d}, 1 \mathrm{H}, J=7.6 \mathrm{~Hz}), 7.12(\mathrm{t}, J=8.6 \mathrm{~Hz}, 1 \mathrm{H}), 5.28-5.25(\mathrm{~m}, 1 \mathrm{H})$, $3.80(\mathrm{~s}, 3 \mathrm{H}), 3.00-2.91(\mathrm{~m}, 2 \mathrm{H}), 1.25-1.18$ (m, 2H); MS (ESI): $m / z 396.4\left(\mathrm{M}^{+}+\mathrm{H}\right)$.

\section{H. pylori IC50 Dose Responses}

H. pylori strains ATCC 43504, SS1, or SS1 NuoD A402P (see below) were grown overnight in brucella broth containing Skirrow's selective medium supplement ${ }^{1}$ and 10\% FBS or brain heart infusion broth containing Skirrow's and 10\% FBS. Cultures were grown in vented $25-\mathrm{cm}^{2}$ tissue culture flasks at $37^{\circ} \mathrm{C}, 10 \% \mathrm{CO}_{2}$ with rotation at 50 rpm. Two-fold serial dilutions of test and control compounds were performed in DMSO or water as appropriate and $1 \mathrm{uL}$ was transferred to a 96 well plate. $99 \mathrm{uL}$ of a 1:10 dilution of an overnight culture of $H$. pylori in Brucella/Skirrow's/10\% FBS was added to each well. Plates were incubated for $18-20 \mathrm{~h}$, and $5 \mathrm{ul}$ of $3 \mathrm{mM}$ resazurin was added to each well. Plates were incubated for an additional 3-5 h, and resazurin reduction was measured using a BioTek Synergy Mx (BioTek, Winooski, VT) with an excitation wavelength of $540 \mathrm{~nm}$ and emission read at $590 \mathrm{~nm}$. Percent inhibition compared to growth controls were calculated and the 50\% inhibitory concentration (IC50) was determined using XLFit software (IDBS, London, United Kingdom).

\section{Cytotoxicity Assays}

FaDu (ATCC HTB-43) and HepG2 (ATCC HB-8065) cell lines were routinely maintained according to the protocols provided by the American Type Culture Collection (ATCC). For cytotoxicity IC-50 determination, 100uL of cell suspension in Eagle's Minimal Essential Medium supplemented with 10\% FBS was seeded into black sided, tissue culture treated 96 well plates (Corning 3603) at a concentration of $3 \times 10^{5}$ cells $/ \mathrm{ml}$. Plates were incubated for 24 hours in a $37^{\circ} \mathrm{C}, 5 \% \mathrm{CO}_{2}$ incubator to allow for cell adhesion and growth. Spent media was aspirated from the test plates and serial two-fold dilutions of test compounds mixed with fresh EMEM $+10 \%$ FBS were added to the plates at $100 \mu \mathrm{L}$ per well. The final DMSO concentration was $1 \%$. The plates were incubated for 72 hours. The media was aspirated and FBS-free EMEM and resazurin (final concentration $0.15 \mathrm{mM}$ ) was added to the plate. Plates were incubated for 3-5 hours and then resazurin reduction was measured using a BioTek Synergy Mx (BioTek, Winooski, VT) with an excitation wavelength of 540nm and emission read at 590nm. Percent inhibition compared to growth controls were calculated and the 50\% inhibitory 
concentration (IC50) curves were generated using XLFit software (IDBS, London, United Kingdom).

\section{Generation of Resistant Mutants, Sequencing and Target Identification}

Mutants resistant to the thienopyrimidine series were generated in two separate ways. Strain HM-274 was chosen for these studies, since it had been previously annotated. Spontaneous mutants of HM-274 were selected by concentrating and harvesting susceptible wildtype cultures and spreading them onto plates containing $2 \mathrm{X}$ MIC of 28 . Two stably resistant isolates were recovered which had MICs 8-fold higher than the wildtype. H. pylori ATCC 43504 was serially passaged onto plates containing increasingly higher concentrations of 1 until the strain was able to grow at $8 \mathrm{X}$ the MIC of the wild-type. The DNA from this resistant mutant was then purified and transformed into strain HM-274 and resistance was confirmed. Genomic DNA was purified from the three resistant mutants as well as HM-274 wildtype using ZR Fungal/Bacterial DNA MiniPrep (Zymo Research) according to the manufacturer's instructions. Whole genome sequencing was performed by Tufts Medical Center. Genome annotation was accomplished using RAST and single nucleotide polymorphism (SNP) identification was performed with CLC Workbench. The two spontaneous resistant mutants had SNPs in nuoD which caused one amino acid change, either A402P or T400I. The serial passage mutant had multiple non-synonymous mutations in nuoD. There were no other shared mutations in all three strains.

Seven additional spontaneous resistant mutants were isolated. Genomic DNA was purified from each and nuoD was amplified by PCR. The PCR products were sequenced and compared to wildtype nuoD. All seven had mutations in nuoD resulting in an amino acid change, either A402P or T400I.

In order to ensure that no secondary mutations were causing thienopyrimidine resistance, a wild-type copy of nuoD was modified to contain the A402P mutation using the Q5 Site Directed Mutagenesis kit (New England Biolabs) according to the manufacturer's instructions. The mutation was confirmed by PCR and then wild-type SS1 was transformed with this plasmid and selected for on plates containing $\mathbf{2 8}$. The A402P mutation in nuoD conferred resistance in strain SS1. Several colonies were chosen and the mutation was confirmed by PCR and sequencing. An SS1 isolate with the NuoD A402P mutation was used in further testing to confirm on target activity of the thienopyrimidine series (see above).

\section{ex vivo Efficacy Study}

This model was modified from what has previously been described ${ }^{2-4}$. Briefly, 6to 8-week-old female C57BL/6 (specific-pathogen-free) mice were obtained from

Charles River Laboratories. Five mice were housed per cage and allowed to acclimate for 
at least $72 \mathrm{~h}$. Animals were inoculated by oral gavage, three times within one week with $0.2 \mathrm{ml}$ of an $H$. pylori SS1 suspension $\left(\sim 10^{9} \mathrm{CFU} /\right.$ dose $)$. The infection was allowed to stabilize for one to two weeks after the final gavage. Mice were euthanized and the stomachs were excised, bisected laterally into equal halves along the long curvature, and washed three times with sterile PBS. Each half was then added to the well of a 12 well plate containing Brucella broth supplemented with 10\% FBS and Skirrow's with or without 100X the MIC of Compound 25. Each half from individual stomachs were assigned to a control and treatment group. After $24 \mathrm{~h}$, the stomach halves were washed, weighed, homogenized, serially diluted, and plated on selective medium. These plates were incubated for 7 days and then colonies were counted to determine colony counts. Data was log transformed and statistical significance was determined using a MannWhitney test with GraphPad Prism software. A P-value less than 0.05 was considered significant. The study was repeated to ensure reproducibility. Animal use complied with the Animal Welfare Act, the Guide for the Care and Use of Laboratory Animals, and the Office of Laboratory Animal Welfare.

\section{Homology Modeling of $H$. pylori's Complex I}

A homology model of the subunits NuoD and NuoB of NADH:ubiquinone Oxidoreductase in Helicobacter pylori was created using Schrodinger's Maestro program. The homology model was built using the protein sequence from a Thermus thermophilus crystal structure (PDB 2FUG) as a template to build the NuoD and NuoB subunits of NADH:quinone oxidoreductase in H. pylori. A series of thienopyrimines and two known complex I inhibitors were docked using Schrodinger's Induced Fit docking based upon the quinone binding channel, based on previous Thermus thermophilus and mitochondrial studies of ubiquinone binding (Tyr85, Arg40, Val403, and Asn401), and from a ligand in our thienopyrimidine series (T400 and A402). ${ }^{7}$ All residue numbers are based on the protein sequence of $H$. pylori. A multiple sequence alignment was performed with mouse, bovine, human, A. fumagatis, H. pylori, E. coli and $M$. tuburculosis using CLC Genomic Workbench 11.

\section{Results and Discussion}

New compounds were synthesized to develop the SAR around the two thienopyrimidine hits as outlined in Scheme 2-1. ${ }^{125-128}$ First, phenylacetaldehyde 3 is reacted with ethyl 3-cyanopropanoate $\mathbf{4}$ to give phenylthiophene $\mathbf{5}$. Heating $\mathbf{5}$ in the excess of formamide formed pyrimidone 6. Chlorination and dehydration of the pyrimidone with phosphoryl chloride afforded the chlorothienopyrimidine 7, which was coupled with a variety of primary and a secondary amine to give targeted 4-position substituted $H$. pylori inhibitors (8-30). These amines were selected based on their structural resemblance to the amine substituents of the hit compounds and the progress of the anti-H. pylori SAR. Initial SAR efforts focused on exploring the 4-position of the thienopyrimidine core, examining the $\mathrm{N}$-alkyl hydroxyl moiety of hit compound $\mathbf{1}$, Series 
Scheme 2-1. Synthesis of thienopyrimidine derivatives.
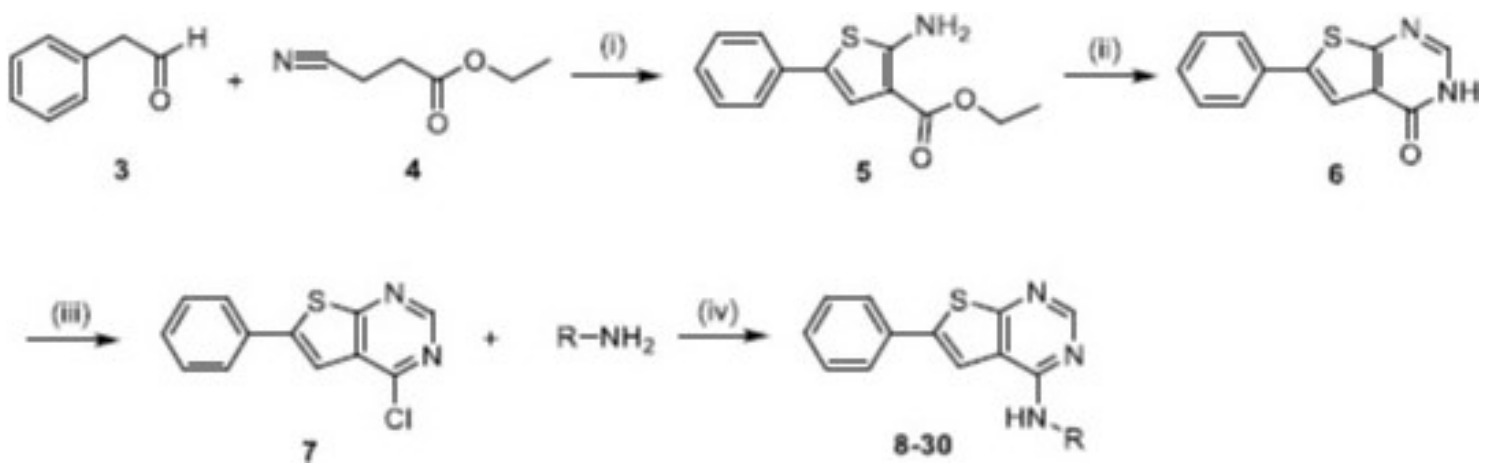

Reagents and conditions: (i) Sulfur, Morpholine, Ethanol, $70^{\circ} \mathrm{C}$, Microwave, $20 \mathrm{~min}$, 90\%; (ii) Formamide, reflux, $18 \mathrm{~h}, 80 \%$; (iii) $\mathrm{POCl}_{3}, \mathrm{~N}, \mathrm{~N}$ dimethylaniline, reflux, $14 \mathrm{~h}$, $90 \%$; (iv) Ethanol, $150^{\circ} \mathrm{C}$, Microwave, 1 h, $80-93 \%$. 
1 (Table 2-1) and phenethyl amine motif of compound 2, Series 2 (Table 2-2). These sidechains were then successful merged into Series 3 (Table 2-3). Commercially available amine starting materials with hydroxyl and aromatic moieties, similar to the moieties identified with the hit compounds, were used to synthesize analogs in these series to investigate the SAR at the 4-position.

In series 1, thienopyrimidines variation to the propyl alcohol like substituent of hit compound $\mathbf{1}$ were examined (Table 2-1). Compound $\mathbf{8}$ was generated first to investigate the effect of N-methylation of the amine group attached at the 4-position of the thienopyrimidine found in hit compound 1. Compound $\mathbf{8}$ demonstrates less anti-H. pylori activity and also increases the lipophilicity negatively impacting the Lipophilic Efficiency (LLE), suggesting that the free secondary amine is preferred in this series. To optimize the spacer between the hydroxyl group and the amine moiety, we synthesized compound 9 with an ethyl motif as spacer. Compared to hit compound 1,9 demonstrates less activity compared to compound $\mathbf{1}$, suggesting that a propyl linker is preferred for improved potency. To investigate branching between the amine moiety at the 4-position and the hydroxyl group of compound 1, we synthesized methyl branched isomers $\mathbf{1 0}$ and 11. Compound 10 demonstrates higher potency and selectivity compared to compound 11, suggesting further SAR development are preferred at the $\alpha$-carbon to the amine over the $\beta$ carbon. The methylation creates a chiral center, the contribution of each isomer in 10 was examined through the synthesis of enantiomers $12(S)$ and $\mathbf{1 3}(R)$. The $R$ enantiomer 13 demonstrates 75 -fold increased activity over the $S$ enantiomer $\mathbf{1 2}$. To examine the contribution of the hydroxyl group, we synthesized compound 14, the Omethylated analog of $\mathbf{1 0}$. Compared to compound 10, 14 demonstrates less potency with a large decrease in LLE, suggesting that the free hydroxyl group is desired for optimal activity. To expand on the linker region, we synthesized isomeric compounds 15, 16 and 17 with an additional methylene unit. Compound 16, $\alpha$-carbon ethyl substituted analog, demonstrates the highest potency of these 3 isomers consistent with previously developed SAR. Compound 18, with a bulkier isopropyl substitution over the ethyl motif found in 16, showed much decreased potency. Overall, within this compound series clear SAR was observed consistent with the analogs engaging a defined molecular target.

Next compounds we examined how phenethyl sidechain of compound 2 performs on the 6-phenyl- thienopyrimidine core found in the first series, four analogs with varying phenyl substitutions were designed and synthesized 19, 20, 21 and 22 (Table 2-2). Best among these compounds, 19 displays the excellent potency and selectivity against $H$. pylori. 19 is the direct phenyl- thienopyrimidine hybrid of $\mathbf{2}$ with the 3,4dimethoxyphenethyl side chain. Substitution of the dimethoxyphenyl motif with dimethylphenyl, compound 20, decrease comparative potency and increased lipophilicity. 3 and 4 substituted pyridyl analogs (21 and 22) designed to increase metabolic stability and solubility also showed substantially decreased potency, suggesting the dimethoxyphenylethyl substitution provides optimum anti-H. pylori activity. To determine the optimum spacer for the phenyl moiety, we synthesized compound $\mathbf{2 3}$ with a methylene motif as spacer. $\mathbf{2 3}$ demonstrates less potency compared to 19, suggesting the ethyl motif is preferred as spacer for this series of compounds. 
Table 2-1. The structure-activity relationship of thienopyrimidines of series 1.

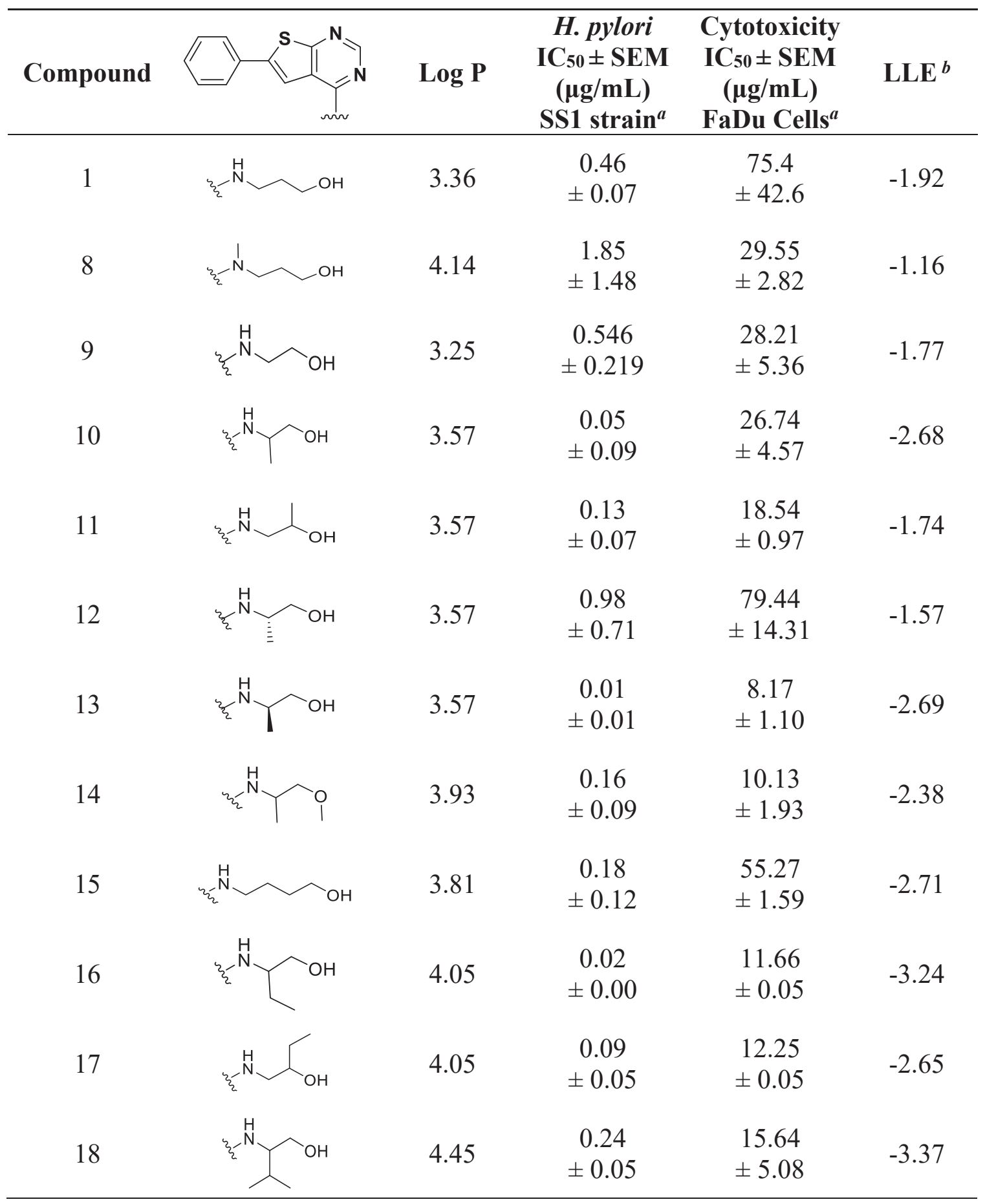

${ }^{a} \mathrm{All} \mathrm{IC}_{50}$ data are the mean of 3 measurements. ${ }^{b} \mathrm{LLE}=\mathrm{pIC}_{50}-\log \mathrm{P}$. 
Table 2-2. The structure-activity relationship of thienopyrimidines of series 2.

\begin{tabular}{|c|c|c|c|c|}
\hline Compound & $\log P$ & $\begin{array}{c}\text { H. pylori } \\
\text { IC50 } \pm \text { SEM } \\
(\mu \mathrm{g} / \mathrm{mL}) \\
\text { SS1 strain }{ }^{a} \\
\end{array}$ & $\begin{array}{c}\text { Cytotoxicity } \\
\text { IC50 } \pm \text { SEM } \\
(\mu \mathrm{g} / \mathrm{mL}) \\
\text { FaDu Cells }{ }^{a}\end{array}$ & $\mathbf{L L E}^{b}$ \\
\hline 19 & 5.53 & $\begin{array}{c}0.02 \\
\pm 0.002\end{array}$ & $\begin{array}{r}133.34 \\
\pm 57.73\end{array}$ & -4.69 \\
\hline 20 & 6.75 & $\begin{array}{c}0.22 \\
\pm 0.17\end{array}$ & $\begin{array}{l}<6.25 \\
\pm 0.00\end{array}$ & --5.83 \\
\hline 21 & 4.44 & $\begin{array}{c}0.48 \\
\pm 0.32\end{array}$ & $\begin{array}{l}29.32 \\
\pm 4.81\end{array}$ & -3.32 \\
\hline 22 & 4.44 & $\begin{array}{c}1.08 \\
\pm 0.47\end{array}$ & $\begin{array}{c}30.31 \\
\pm 0.64\end{array}$ & -2.72 \\
\hline 23 & 5.25 & $\begin{array}{c}0.237 \\
\pm 0.167\end{array}$ & $\begin{array}{c}9.72 \\
\pm 3.00\end{array}$ & -3.91 \\
\hline
\end{tabular}

${ }^{a}$ All $\mathrm{IC}_{50}$ data are the mean of 3 measurements. ${ }^{b} \mathrm{LLE}=\mathrm{pIC}_{50}-\log \mathrm{P}$. 
Table 2-3. The structure-activity relationship of thienopyrimidines of series 3 .

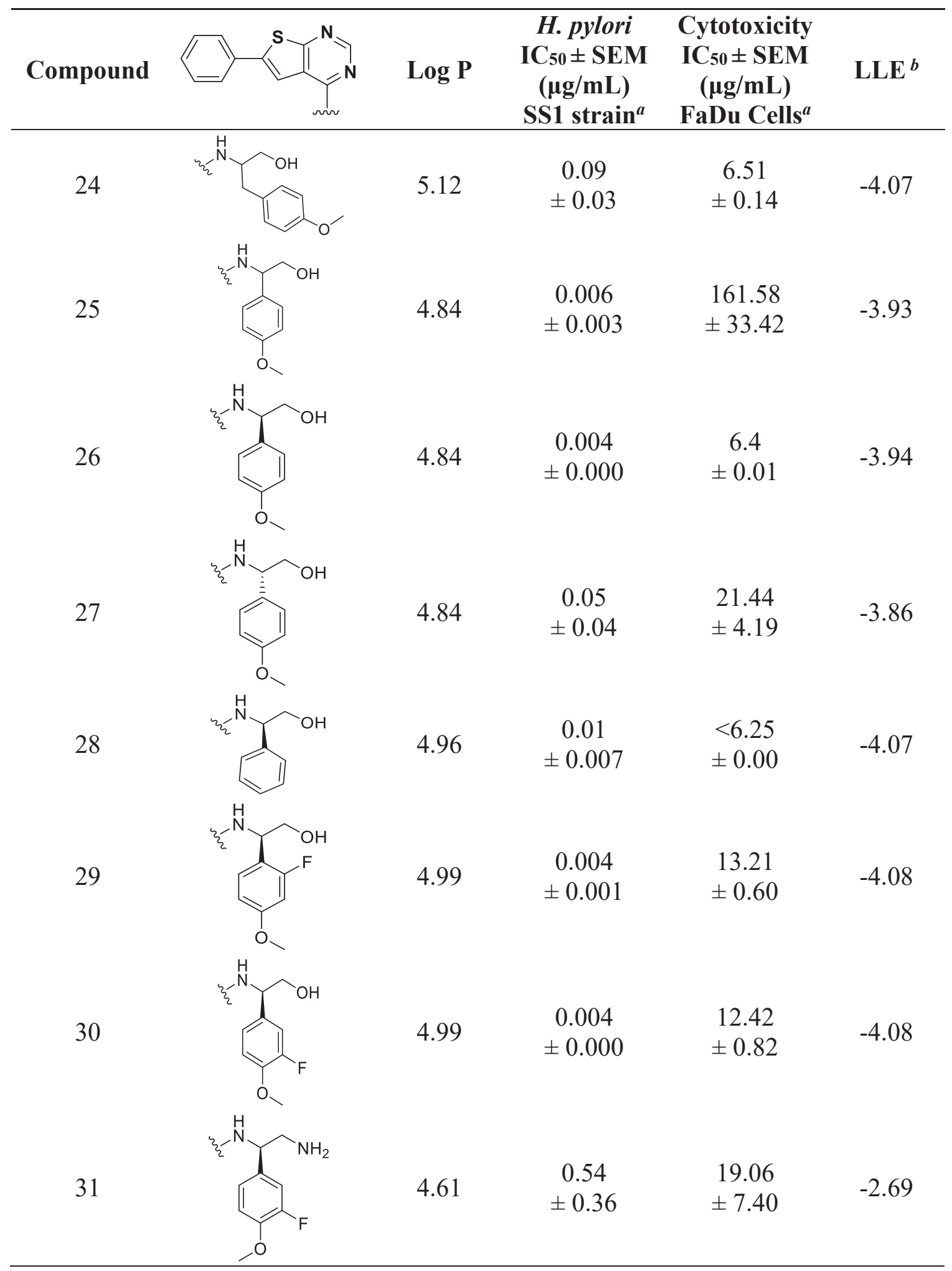

${ }^{a}$ All $\mathrm{IC}_{50}$ data are the mean of 3 measurements. ${ }^{b} \mathrm{LLE}=\mathrm{pIC}_{50}-\log \mathrm{P}$. 
The SAR progression revealed series leads 16 and 19 from hits 1 and $\mathbf{2}$ respectively. We hypothesized that merging both substituents at the 4-position of phenylthienopyrimidine could produce analogs with increased potency. Thus, we synthesized compound 24 (Table 2-3) which demonstrated higher activity than 16 and similar potent activity to 19. In attempt to optimize the activity of $\mathbf{2 4}$ we were able to purchase several 2-phenylglycinol building block with a shorter spacer between the aryl motif and the nitrogen. First compound $\mathbf{2 5}$ was generated which demonstrates the highest potency of the series, in low ng/mL-range of activity (H.p. $\left.\mathrm{IC}_{50}=0.006 \mu \mathrm{g} / \mathrm{ml}\right)$. Both enantiomers of 25 were then synthesized. Compound 26, the R enantiomer of 25, showed higher potency (H.p. $\mathrm{IC}_{50}=0.004 \mu \mathrm{g} / \mathrm{ml}$ ) than the S enantiomer (27). To further understand the SAR around the phenylglycinol aryl ring, we synthesized fluorinated compounds, 28 and 29. These compounds demonstrated comparable potency to $\mathbf{2 6}$.

While various aryl rings at the 4-position of compound 28-30 demonstrate activity, we synthesized primary amine analog $\mathbf{3 1}$ to further elucidate the importance of the hydroxyl moiety and the SAR at the 4-position, as primary amines have been shown to enhance uptake of drugs into Gram-negative bacteria. ${ }^{129}$ Compound $\mathbf{3 1}$ was synthesized as described in Scheme 2-2. ${ }^{143,144}$ Amine compound 32 was protected with di-tert-butyl dicarbonate to yield the the boc-protected amine 33. Substitution of the hydroxyl group of $\mathbf{3 3}$ with a phthalamide group was carried out under mitsunobu conditions to give the intermediate 34. Next, the removal of the boc group yielded the amine 35, which was coupled to chlorothienopyrimidine 7 to afford the intermediate 36 . In the final step, the deprotection of the phthalamide group of $\mathbf{3 6}$ afforded the targeted thienopyrimidine 31. However, 31 did not show improved activity compared to 30, suggesting the hydroxyl moiety is required for the anti-H. pylori activity.

Initial hits $\mathbf{1}$ and $\mathbf{2}$ also differ in their substitution patterns at -C5 and C6 of the thienopyrimidine ring. Compounds 37-40 were synthesized as described in Scheme 2-3. Commercially available chlorothienopyrimidines (41-44) were used in the coupling reaction with the optimized phenylglycinol sidechain to yield final compounds (37-40). The effect of substitution at these positions was then studied in combination with the optimized phenylglycinol 4-postion sidechain. Compounds 37, 38, 39 and 40 (Table 2-4) were generated and their activities were compared to compound 28 (Table 2-3). Moving the phenyl ring to the 5-position (39 and 40) substantially reduced anti-H. pylori potency. The dimethyl analog $\mathbf{3 0}$ with a similar substitution pattern of compound $\mathbf{2}$, showed $a$ slight improvement over $\mathbf{2}$ but weaker activity than the 6-phenyl substituted analog $\mathbf{3 8}$. 38 with a similar phenyl substitution pattern to series 3 compounds and an addition methyl substitution, demonstrates comparable activity to $\mathbf{2 8}$, suggesting the phenyl ring at the 6position optimal and 5-position can be hydrogen and methyl but not a larger phenyl group.

To determine the target of the thienopyrimidines, resistant $H$. pylori mutants were successfully isolated by serial passage on agar medium containing compound $\mathbf{2 5}$. Upon whole genome sequencing, two spontaneous mutants were identified containing nonsynonymous point mutations in $n u o D$. The resulting amino acid substitutions were T400I 


\section{Scheme 2-2. Synthesis of amine 31 .}
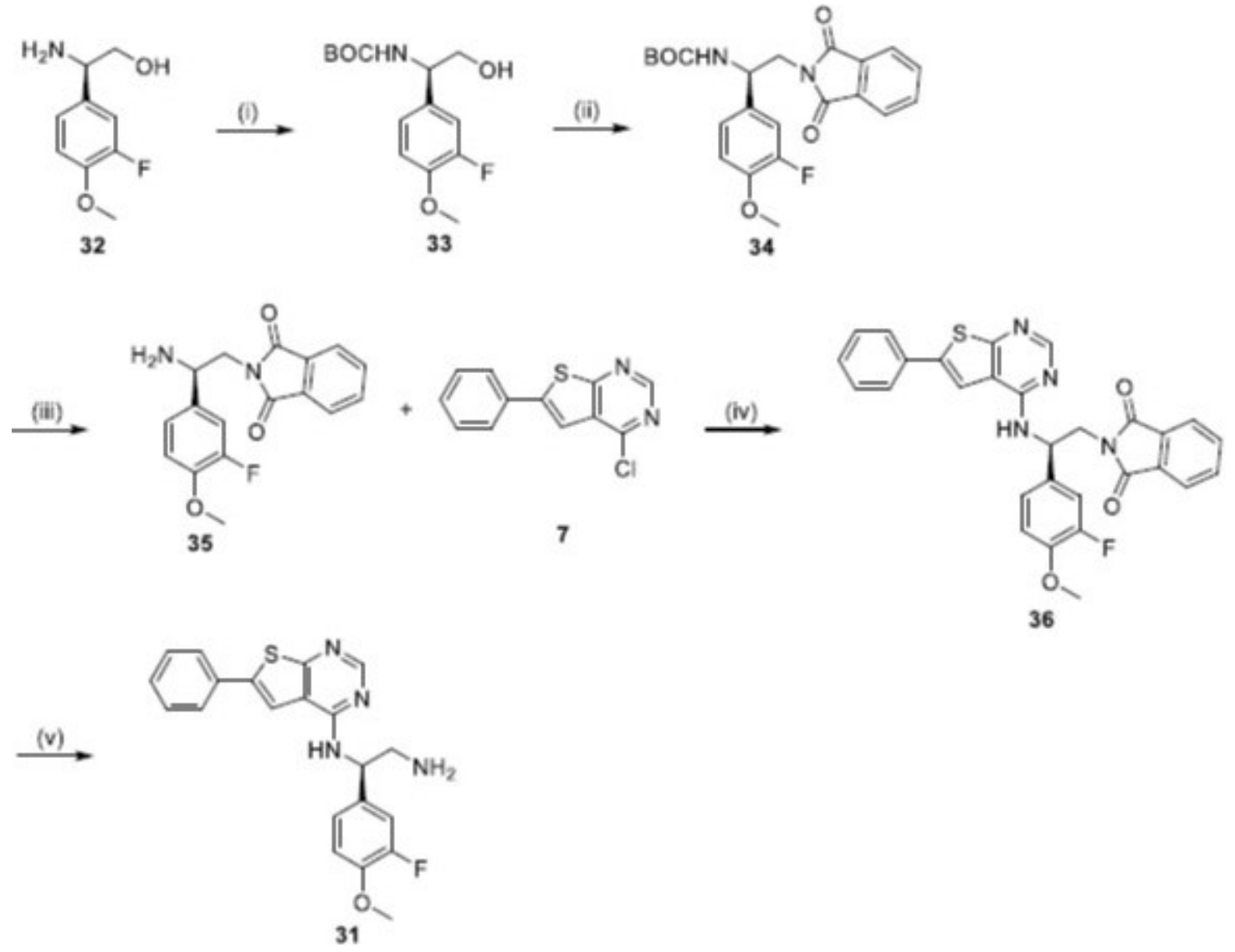

Reagents and conditions: (i) di-tert-butyl dicarbonate, Et 3 N, DCM, RT, 2 h; (ii) phthalimide, DIAD, $\mathrm{Ph}_{3} \mathrm{P}, \mathrm{DCM}, \mathrm{RT}, 4 \mathrm{~h}$; (iii) $4 \mathrm{M} \mathrm{HCl}$ in dioxane, DCM, methanol, RT, 4 h; (iv) Et $3 \mathrm{~N}$, reflux, 6 h; (v) $\mathrm{NH}_{2} \mathrm{NH}_{2}$, methanol, RT, 6 h. 
Scheme 2-3. Synthesis of thienopyrimidine analogs with modifications at the 5 and 6-position.
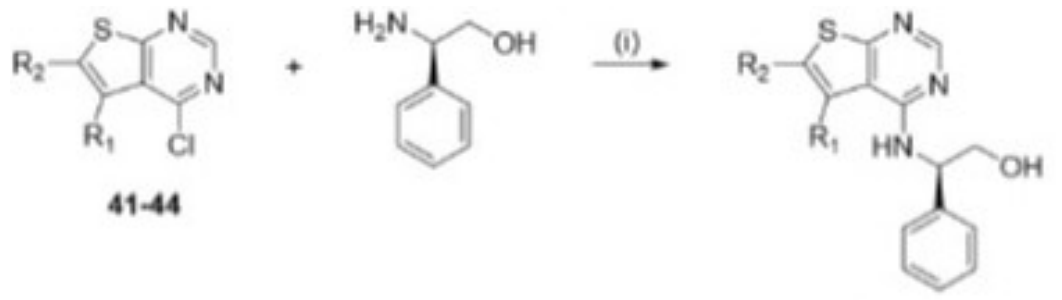

$37-40$

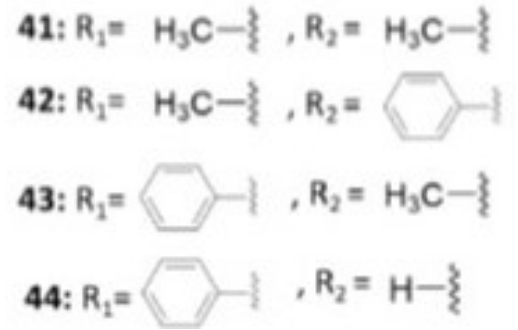

37: $\mathrm{R}_{1}=\mathrm{H}_{3} \mathrm{C}-\left\{, \mathrm{R}_{2}=\mathrm{H}_{3} \mathrm{C}-\right\}$

38: $\mathrm{R}_{1}=\mathrm{H}_{3} \mathrm{C}-\left\{, \mathrm{R}_{2}=\square\right.$

39: $\mathrm{R}_{1}=\square, \mathrm{R}_{2}=\mathrm{H}_{3} \mathrm{C}-\boldsymbol{\}}$

40: $\mathrm{R}_{1}=\square-\mathrm{R}_{2}=\mathrm{H}-\xi$

Reagents and conditions: (i) $\mathrm{Et}_{3} \mathrm{~N}, 150^{\circ} \mathrm{C}$, Microwave, $1 \mathrm{~h}, 89-93 \%$. 
Table 2-4. The structure-activity relationship of series 4 with substitutions at the 5 and 6-positions of the thienopyrimdine core.

\begin{tabular}{|c|c|c|c|c|c|c|}
\hline Compound & $\mathbf{R}_{\mathbf{1}}$ & $\mathbf{R}_{2}$ & $\log P$ & $\begin{array}{c}\text { H. pylori } \\
\text { IC }_{50} \pm \mathrm{SEM} \\
(\mu \mathrm{g} / \mathrm{mL}) \\
\text { SS1 strain } \\
\end{array}$ & $\begin{array}{c}\text { Cytotoxicity } \\
\text { IC }_{50} \pm \text { SEM } \\
(\mu \mathrm{g} / \mathrm{mL}) \\
\text { FaDu Cells }{ }^{a}\end{array}$ & $\mathbf{L L E}^{b}$ \\
\hline 37 & $\mathrm{Me}-\xi$ & $\mathrm{Me}-\xi$ & 4.05 & $\begin{array}{c}0.25 \\
\pm 0.18\end{array}$ & 24.61 & -1.84 \\
\hline 38 & $\mathrm{Me}-\xi$ & & 5.45 & $\begin{array}{c}0.01 \\
\pm 0.001\end{array}$ & 447.64 & -4.51 \\
\hline 39 & & $\mathrm{Me}-\xi$ & 5.24 & $\begin{array}{c}10.24 \\
\pm 13.59\end{array}$ & 4.37 & -1.7 \\
\hline 40 & & $\mathrm{H}-\xi$ & 4.91 & $\begin{array}{c}0.21 \\
\pm 0.16\end{array}$ & 60.69 & -2.81 \\
\hline
\end{tabular}

${ }^{a} \mathrm{All} \mathrm{IC}_{50}$ data are the mean of 3 measurements. ${ }^{b} \mathrm{LLE}=\mathrm{pIC}_{50}-\log \mathrm{P}$. 
and A402P (Figure 2-2). The gene, nuoD encodes for the D subunit of NADH:Quinone Oxidoreductase, also known as the respiratory Complex I (Figure 2-3A). ${ }^{109,} 136-138$ These results suggest that Complex I, a protein that catalyzes the first step of oxidative phosphorylation, is the putative target of thienopyrimidines. ${ }^{107,139}$

To further understand the inhibition of H. pylori's Complex I with the thienopyrimidine 25, a homology model of the wildtype subunits NuoD and the neighboring NuoB was generated using Thermus thermophilus' Complex I as a template. ${ }^{20}$ Using Clustal Omega it was found that the NuoB subunit has $59 \%$ homology with T. thermophilus' complex I subunit Nqo6 and 42\% homology with $T$. thermophilus' complex I subunit Nqo4. The T400I and A402P mutations in NuoD are found in the quinone binding pocket indicating the thienopyrimidine series bind in this pocket. In order to visualize the possible binding, the chiral thienopyrimidine $\mathbf{2 6}$ was docked into the known menaquinone binding pocket at the NuoB-NuoD interface (Figure 2-3B). This model suggests that thienopyrimidine $\mathbf{2 6}$ forms a hydrogen bond with the backbone of T400 and suggests the small molecule inhibits menaquinone binding. In support of the computational studies, the thienopyrimidine $\mathbf{2 6} \mathrm{IC}_{50}$ data shows toxicity to both wild type and the A402P mutant $H$. pylori. Further details of the target identification studies and the homology modeling are provided in the Experimental section of chapter II. To validate that the thienopyrimidines are on target, all analogs were tested against the mutant $H$. pylori strain (SS1 nuoD A402P). The $\mathrm{IC}_{50}$ values against the mutant strain are provided in Tables A-1 and A-2. The thienopyrimidines are found generally inactive against the mutant strain, further supporting that Complex I is the target of the series.

Given compound 25 demonstrates high in vitro potency (H.p. $\mathrm{IC}_{50}=0.006 \mu \mathrm{g} / \mathrm{ml}$, Table 2-3), we evaluated the in vitro ADME and in vivo pharmacokinetic properties. ${ }^{22-24}$ The results of these studies are provided in Tables A-3 and A-4, respectively. Compound 25 showed good microsomal stability $\left(\mathrm{t}_{1 / 2}=3.8 \mathrm{hr}\right.$ ) and permeability (Ave $\mathrm{Pe}=239.5 \mathrm{x}$ $10^{-6} \mathrm{~cm} / \mathrm{s}$ ) but it is highly bound to human plasma protein (HPPB \%=99). 25 showed good in vivo exposure (AUC $=31907.71 \mathrm{hr} * \mathrm{ng} / \mathrm{mL}$, Table A-3) at $50 \mathrm{mg} / \mathrm{kg}$, i.p. dose. The drug concentration of $\mathbf{2 5}$ in the plasma was above its $\mathrm{IC}_{50}$ value for more than 8 hours. With good plasma exposure and cytotoxicity profile, we proceeded to test $\mathbf{2 5}$ in the H. pylori murine infection model. ${ }^{133,134}$ Further details are provided in (Figure A-1). Compared to the BMT (Bismuth, Metronidazole and Tetracycline) triple therapy as control, 25 did not show efficacy at $50 \mathrm{mg} / \mathrm{kg}$ (OG), $50 \mathrm{mg} / \mathrm{kg}$ (i.p) or $25 \mathrm{mg} / \mathrm{kg}$ (i.p). ${ }^{135}$ To investigate the lack of in vivo efficacy, we tested compound $\mathbf{2 5}$ in the ex vivo model of H. pylori infection. Ten mice were infected with $H$. pylori SS1 strain and sacrificed after three days to harvest the stomachs. ${ }^{134,140}$ In a 12 well plate, one half of each stomach was placed in Brucella-FBS medium containing compound 25 and the other half was placed in Brucella-FBS medium only and treated as control. Further details of the ex vivo experiment are described in the experimental section of chapter II. As described in 


\begin{tabular}{|c|c|}
\hline & 391 \\
\hline mut2_genome & AVTVIGSTNPVFGEVDR \\
\hline mut2_PCR & AVTVIGSTNPVFGEVDR \\
\hline mut1_genome & AVTVIGS INAVFGEVDR \\
\hline mut1_PCR & AVTVIGS INAVEGEVDR \\
\hline WT_nuoD_genome & AVTVIGSTNAVEGEVDR \\
\hline
\end{tabular}

Figure 2-2. Sequence alignment of the wildtype nuoD and two $H$. pylori mutant nuoD.

Resistant mutants were generated by exposing $H$. pylori to compound $\mathbf{2 5}$ on agar plate. Whole genome sequencing was performed, and single nucleotide polymorphism analysis identified two spontaneous mutants, which had non-synonymous point mutations in nuoD. The resulting amino acid changes were Threonine to Isoleucine (T400I) and Alanine to Proline (A402P). nuoD encodes for the subunit D of NADH:Quinone Oxidoreductase also known as Complex I, a membrane protein that catalyzes the first step of oxidative phosphorylation. Complex I is the putative target of the thienopyrimidine. 
A.

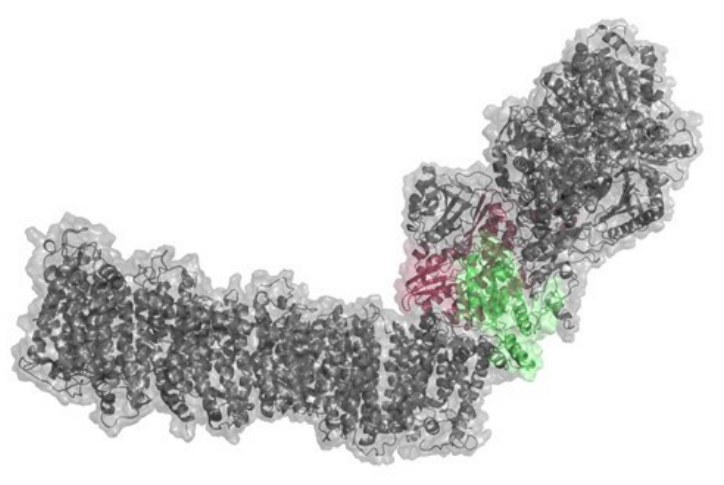

B.

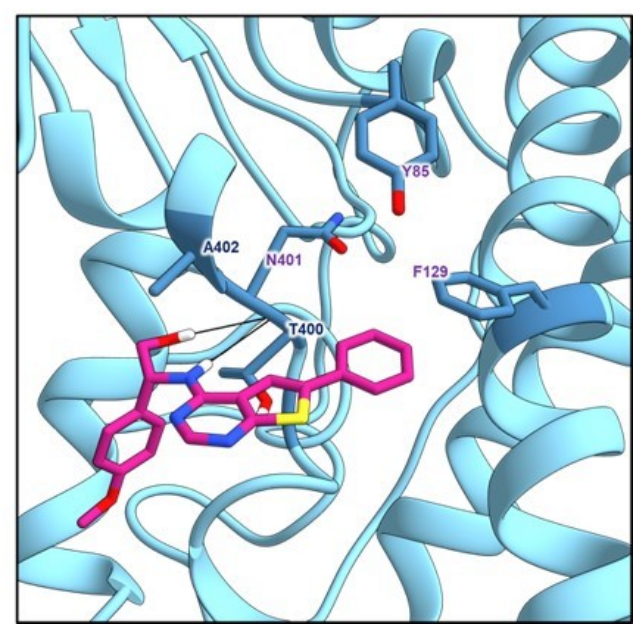

Figure 2-3. The homology model of $\boldsymbol{H}$. pylori's respiratory Complex I, the putative target of the thienopyrimidines.

(A) The structure of Complex I with NuoD colored in dark red. The homology model was generated using the crystal structure of Thermus Thermophilus' Complex I. The thienopyrimidines are predicted to bind at the interface of NuoD and NuoB. (B) The lead compound $\mathbf{2 6}$ docked in the proposed binding pocket of Complex 1 . The quinone binding residues are labeled in purple and the substituted amino acids in resistant mutants (T400I and A402P) against the thienopyrimidine $\mathbf{2 6}$ are labeled in blue. According to the homology model, the changed amino acids are located near the quinone binding pocket, suggesting 26 inhibits the endogenous quinone from binding to Complex I. 
Figure 2-4, 25 substantially eradicates $H$. pylori, with 6 stomachs found below the limit of detection (Figure 2-4). Compound 25 eradicates $H$. pylori in the ex vivo settings but does not show in vivo efficacy. This discrepancy suggests that not enough of $\mathbf{2 5}$ is getting to the site of infection and the optimization of the pharmacokinetic properties is required for the series to demonstrate in vivo efficacy.

\section{Conclusion}

In conclusion, we have synthesized 27 analogs and established the anti-H. pylori SAR at the 4, 5 and 6 position of the thienopyrimidine core. These efforts have generated potent compounds, including the lead compound 25, with the $\mathrm{IC}_{50}$ of $0.006 \mu \mathrm{g} / \mathrm{mL}$.

Although 25 does not show in vivo efficacy, it eradicates $H$. pylori in the ex vivo model. The mode of action studies identified H. pylori's respiratory Complex I as the putative target of the thienopyrimidines, with the amino acid changes found in the NuoD subunit. Complex I is located in the plasma membrane of bacteria and it catalyzes the first step of oxidative phosphorylation. Targeting oxidative phosphorylation has been done safely in the clinic, as exemplified by metformin, a drug used for the treatment of diabetes. ${ }^{141}$ Additionally, targeting oxidative phosphorylation is becoming an attractive approach in the treatment of relapsed/refractory AML and solid tumors. ${ }^{142}$ Recent knockout mutagenesis studies suggest that nuoD is an essential gene for H. pylori but not for the majority of other bacteria, indicating that $H$. pylori can be targeted selectively without perturbation the host microbiota. ${ }^{16,31}$ These examples suggest that Complex I is a worthwhile target in eradicating H. pylori. 25 is a promising lead compound that is worth further optimization to realize in vivo efficacy in the $H$. pylori infected mouse model. 


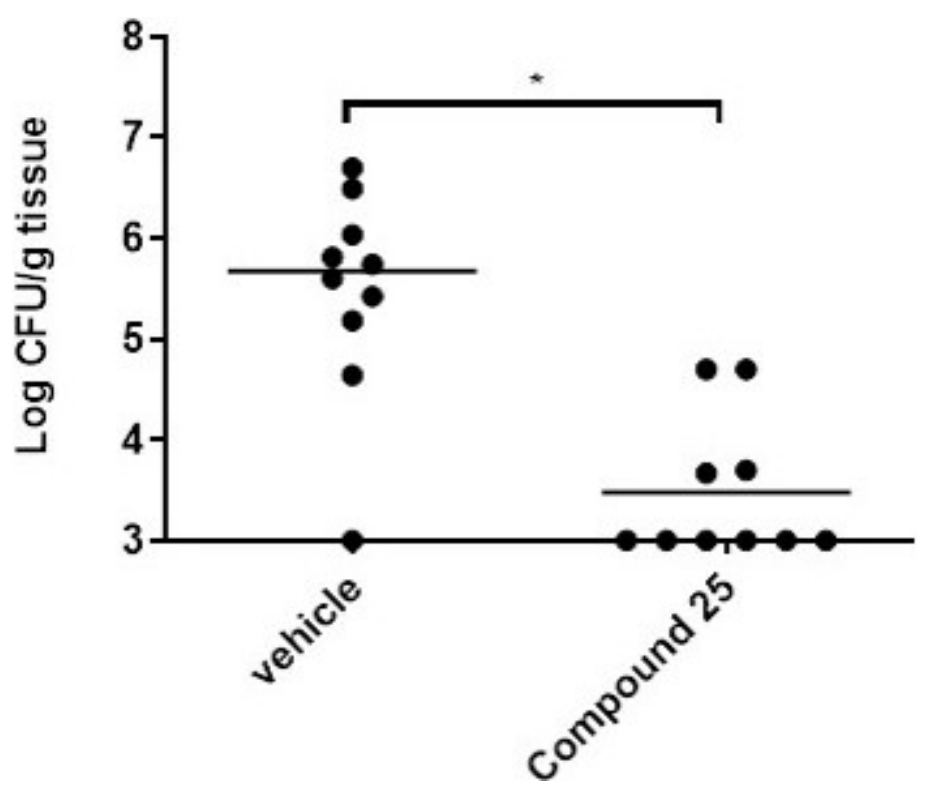

Figure 2-4. Compound 25 eradicates $H$. pylori in the ex vivo efficacy experiment. Ten mice were infected with $H$. pylori. After 3 days, stomachs were harvested and cut in half. In a 12 well plate, one half was placed in Brucella-FBS medium and the other half in Brucella-FBS medium with $0.6 \mu \mathrm{g} / \mathrm{mL}$ of compound 25. After incubation and homogenization, diluted samples were plated for colony counting. 25 eradicates $H$. pylori to below the limit of detection in 6 stomachs. 


\section{CHAPTER 3. ADME OPTIMIZATION OF THE THIENOPYRIMIDINES AGAINST HELICOBACTER PYLORI}

\section{Introduction}

The rate of successful treatment of $H$. pylori infections, with the clarithromycin triple therapy, is around $75 \% .{ }^{1}$ The triple therapy, which consists of a proton pump inhibitor and two broad-spectrum antibiotics such as clarithromycin and amoxicillin, is increasingly becoming ineffective due to the rise of resistance to these antibiotics. ${ }^{26,27,31}$ The World Health Organization lists H. pylori as a high priority drug resistant bacterium. ${ }^{7}$ In a recent study, $22 \%$ of patients tested were not cured by either the triple therapy or the quadruple therapy with Bismuth added. ${ }^{27}$ The triple therapy is also associated with poor patient compliance due to side effects such as diarrhea, derived from the effect of these broad-spectrum antibiotics on the host microbiota. ${ }^{11}$ Therefore, a novel, narrow-spectrum drug is needed for the treatment of $H$. pylori infections, to address the challenges of drug resistance against anti-H. pylori drugs and their side effects.

Advances in whole genome sequencing and highthrough-put screening approaches have provided opportunities to discover novel chemical matter against $H$. pylori. We have previously reported a series of thienopyrimidines that were developed from two hits, discovered through a high through-put screen of 219,197 compounds. Subsequent structure-activity relationship studies of the thienopyrimidines against $H$. pylori, compound 25 (Figure 3-1A) emerged with a high potency (H. pylori $\mathrm{IC}_{50}=0.006$ $\mu \mathrm{g} / \mathrm{mL})$ and tolerable cytotoxicity against the human FaDu cells $\left(\right.$ Cytotox $\mathrm{IC}_{50}=131$ $\mu \mathrm{g} / \mathrm{mL}$ ). Mode of action studies with the lead compound 25 were performed by the generation and sequencing of resistant mutants. These studies revealed the respiratory Complex I as the putative target of the series, with amino acid changes found in the NuoD subunit. NuoD has been previously identified as the target of a series of the benzimidazole compounds by researchers at AstraZeneca and it was found to be essential for $H$. pylori but not for most of bacteria including E. coli. ${ }^{105,106}$ Therefore, NuoD is an attractive target, in eradicating $H$. pylori while preserving the gut microbiota. Complex I, also known as NADH:Quinone Oxidoreductase, catalyzes the first step of oxidative phosphorylation. ${ }^{139}$

The lead compound $\mathbf{2 5}$ eradicates $H$. pylori in the ex vivo model of $H$. pylori infection. However, 25 lacked in vivo efficacy in the $H$. pylori murine model at $50 \mathrm{mg} / \mathrm{kg}$ by oral gavage; suggesting that further optimization of pharmacokinetic properties of the series is required. An examination of the in vivo pharmacokinetic properties, 25 shows good exposure (AUC: 31,907 hr*ng/mL at $50 \mathrm{mg} / \mathrm{kg}$ OG, Figure 3-1A) and a long high half-life in liver microsomes $\left(\mathrm{t}_{1 / 2}=3.8 \mathrm{hr}\right.$, Figure 3-1A) suggesting that hepatic metabolism was not the limiting factor. However, 25 demonstrates a relatively high volume of distribution (Vss $=6.28 \mathrm{~L}$, Figure 3-1A), suggesting 25 is being trapped in fatty tissues despite good exposure. ${ }^{145} 25$ also shows poor solubility (Sol: $0.1 \mu \mathrm{M}$, 
A.

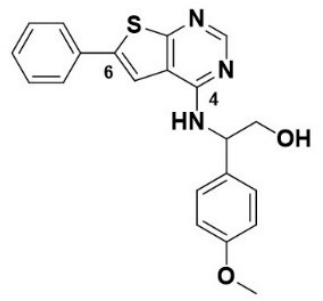

25

H. pylori $\mid \mathrm{IC}_{50}: 0.006 \mu \mathrm{g} / \mathrm{mL}$

Cytotox IC $50: 131 \mu \mathrm{g} / \mathrm{mL}$

ClogP: 4.23

${ }^{a} A U C: 31907.71$ hr*ng/mL

${ }^{a}$ Vss: $6.28 \mathrm{~L} / \mathrm{Kg}$

${ }^{b}$ Micr. Stab. $\left(\mathrm{t}_{1 / 2}\right): 3.8 \mathrm{hr}$

bSol: $0.1 \mu \mathrm{M}$

bНPPB\%: 99.3
B. ii. To introduce fluoro parasubstitution to block metabolism and replace phenyl ring with a pyridinyl, pyrazolyl and a methyl group to improve solubility
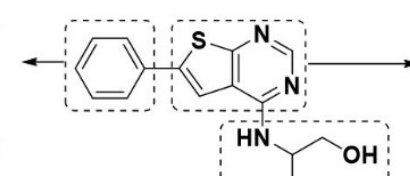

:

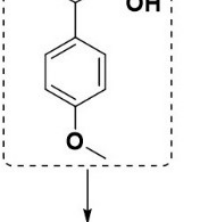

i. To introduce solubilizing amine moieties and to replace para methoxy ring with pyridinyl ring, to improve solubility and to reduce lipophilicity respectively

Figure 3-1. Lead compound 25 and targeted moieties to improve the ADME properties.

(A) ${ }^{\text {a }}$ in vivo pharmacokinetic properties of $\mathbf{2 5}$ at $50 \mathrm{mg} / \mathrm{Kg}$ i.p. and ${ }^{\mathrm{b}} \mathrm{ADME}$ properties of 25. (B) Chemical modifications on targeted moieties of $\mathbf{2 5}$, to improve the ADME properties of the series. 
Figure 3-1A) and is highly bound to plasma protein $(\mathrm{HPPB} \%=99.3$, Figure 3-1A), reducing the amount of free drug able to reach the site of infection. These limitations are compounded by the hard to reach biological niche of $H$. pylori, found in the glands of the stomach and in the mucus layer.

To tackle these shortcomings, in this chapter we look to introduce chemical modifications that will reduce the lipophilicity and increase the solubility while maintaining selective anti-H. pylori activity and resistance to hepatic metabolism of $\mathbf{2 5}$. In this chapter we explore the optimization of $\mathbf{2 5}$ through targeted synthesis and examination of the anti-H. pylori activity and ADME properties of four subseries (Figure 3-1B): (i) We replace metabolically labile and solubility limiting phenyl ring substituted at the 6-poisition of the thienopyrimidine scaffold with pyridinyl, pyrazolyl and methyl motifs; (ii) Through a scaffold hopping exercise, the introduction of core modifications to improve the solubility and reduce the tendency of drug to bind to plasma protein are examined; ${ }^{149}$ (iii) The introduction of primary amine motif to the side chain of $\mathbf{2 5}$, increase Gram-negative drug entry and increase the distribution in the body fluids is also tested; ${ }^{146,147}$ (iv) to reduce drug clearance and compensate for changes elsewhere in the ring, introduce blocking modifications such as para-fluoro-substitution of the 6-phenyl

ring of the thienopyrimidine core are also examined. ${ }^{148}$ Through analysis of the microbiological and pharmacological properties of the new analogs, new lead candidates have emerged suitable for further evaluations.

\section{Materials and Methods}

\section{Synthesis of Amine 46}

tert-butyl (2-hydroxy-1-(4-methoxyphenyl)ethyl)carbamate (60). Di-tert-butyl dicarbonate $(0.447 \mathrm{~g}, 2.047 \mathrm{mmol})$ was added to a solution of 2-hydroxy-1-(4methoxyphenyl)ethan-1-aminium chloride $59(0.347 \mathrm{~g}, 1.706 \mathrm{mmol})$ and triethylamine $(0.286 \mathrm{~mL}, 2.047 \mathrm{mmol})$ in DCM $(5.6 \mathrm{~mL})$. The reaction mixture was stirred for $2 \mathrm{~h}$ at room temperature. The mixture was concentrated and purified by flash column chromatography (Isolera 1, Biotage, $10 \mathrm{~g}$ size, elution gradient $12-80 \%$ ethyl acetate in hexanes). Pure fractions were evaporated to afford $\mathbf{6 0}$ as a white solid.

tert-butyl (2-(1,3-dioxoisoindolin-2-yl)-1-(4-methoxyphenyl)ethyl)carbamate (61). DIAD (0.141 $\mathrm{mL}, 0.729 \mathrm{mmol})$ was added dropwise at room temperature, to a solution of 60 ( $0.150 \mathrm{~g}, 0.561 \mathrm{mmol})$, phthalimide $(0.107 \mathrm{~g}, 0.729 \mathrm{mmol})$ and $\mathrm{Ph}_{3} \mathrm{P}$ $(0.191 \mathrm{~g}, 0.729 \mathrm{mmol})$ in THF $(.6 \mathrm{~mL})$. The resulting mixture was stirred for $16 \mathrm{~h}$ at room temperature. The mixture was extracted with Ethyl Acetate/Water and the organic phase was washed with brine, dried $\left(\mathrm{Na}_{2} \mathrm{SO}_{4}\right)$ and evaporated in vacuo. The residue was purified by flash column chromatography (Isolera 1, Biotage, $10 \mathrm{~g}$ size, elution gradient $12-60 \%$ ethyl acetate in hexanes), to afford 61 as a yellow solid. 
2-(2-amino-2-(4-methoxyphenyl)ethyl)isoindoline-1,3-dione (62) and 2-(2-(4methoxyphenyl)-2-((6-phenylthieno[2,3-d]pyrimidin-4-yl)amino)ethyl)isoindoline-1,3dione (63). $\mathrm{HCl}$ (4 M in 1,4-dioxane, $0.9 \mathrm{~mL}, 3.6 \mathrm{mmol}$ ) was added to a solution of $\mathbf{6 1}$ $(0.300 \mathrm{~g}, 0.757 \mathrm{mmol})$ in a mixture of DCM $(2 \mathrm{~mL})$ and methanol $(1 \mathrm{~mL})$, and the resulting mixture was stirred for $16 \mathrm{~h}$ at room temperature. The mixture was subjected to acid-base extraction with Ethyl Acetate/Water and the organic phase was washed with brine, dried $\left(\mathrm{Na}_{2} \mathrm{SO}_{4}\right)$ and concentrated in vacuo. The resulting residue $\mathbf{6 2}$ (yellow oil) was used in the next step without further purification. $\mathbf{6 3}$ was synthesized following the general procedure for amination of 4-chloro-6-phenylthieno[2,3- $d]$ pyrimidine.

\section{1-(4-methoxyphenyl)-N1-(6-phenylthieno[2,3-d]pyrimidin-4-yl)ethane-1,2-}

diamine (46). Compound 63 ( $0.130 \mathrm{~g}, 0.257 \mathrm{mmol})$ was dissolved in methanol and a 80 $\%$ hydrazine hydrate solution $(0.04 \mathrm{~mL}, 0.642 \mathrm{mmol})$ was added dropwise to the solution at $0^{\circ} \mathrm{C}$. The reaction mixture was stirred for $16 \mathrm{~h}$ at room temperature. The mixture was extracted with Ethyl Acetate/Water and the organic phase was washed with brine, dried $\left(\mathrm{Na}_{2} \mathrm{SO}_{4}\right)$ and evaporated in vacuo. The residue was purified by flash column chromatography (Isolera 1, Biotage, $10 \mathrm{~g}$ size, elution gradient 2-20\% methanol in ethyl acetate) to afford $\mathbf{4 6}$ as a white solid.

\section{1-(4-methoxyphenyl)-N1-(6-phenylthieno[2,3-d]pyrimidin-4-yl)ethane-1,2-} diamine (46). ${ }^{1} \mathrm{H}$ NMR (DMSO, $\left.400 \mathrm{MHz}\right) \delta 8.32(\mathrm{~d}, J=0.7 \mathrm{~Hz}, 1 \mathrm{H}), 8.03(\mathrm{~s}, 1 \mathrm{H}), 7.77$ $-7.67(\mathrm{~m}, 2 \mathrm{H}), 7.51(\mathrm{dd}, J=8.3,7.0 \mathrm{~Hz}, 2 \mathrm{H}), 7.45-7.35(\mathrm{~m}, 3 \mathrm{H}), 6.97-6.88(\mathrm{~m}, 2 \mathrm{H})$, $5.65(\mathrm{dd}, J=10.6,4.4 \mathrm{~Hz}, 1 \mathrm{H}), 3.70(\mathrm{~s}, 3 \mathrm{H}), 3.32(\mathrm{dd}, J=13.1,10.6 \mathrm{~Hz}, 1 \mathrm{H}), 3.23(\mathrm{dd}, J$ $=13.1,4.5 \mathrm{~Hz}, 1 \mathrm{H})$. MS (ESI): $m / z 377\left(\mathrm{M}^{+}+\mathrm{H}\right)$.

\section{Synthesis of Thienopyrimidine Analog 45}

45 was synthesized following the general procedure for amination of 4-chloro-6phenylthieno[2,3-d]pyrimidine.

(R)-3-(4-methoxyphenyl)-3-((6-phenylthieno[2,3-d]pyrimidin-4yl)amino)propan-1-ol (45). ${ }^{1} \mathrm{H}$ NMR (DMSO, $\left.400 \mathrm{MHZ}\right): \delta 8.27(\mathrm{~d}, J=4.1 \mathrm{~Hz}, 1 \mathrm{H})$, $8.19-8.11$ (m, 2H), $7.74-7.64$ (m, 2H), 7.52 (q, $J=6.4,6.0 \mathrm{~Hz}, 2 \mathrm{H}), 7.41$ (d, $J=8.8$ $\mathrm{Hz}, 1 \mathrm{H}), 7.36-7.30(\mathrm{~m}, 2 \mathrm{H}), 6.91-6.84(\mathrm{~m}, 2 \mathrm{H}), 5.46(\mathrm{~d}, J=7.7 \mathrm{~Hz}, 1 \mathrm{H}), 4.56(\mathrm{t}, J=$ $4.7 \mathrm{~Hz}, 1 \mathrm{H}), 3.71(\mathrm{~d}, J=3.2 \mathrm{~Hz}, 2 \mathrm{H}), 3.46(\mathrm{dd}, J=13.0,6.6 \mathrm{~Hz}, 2 \mathrm{H}), 2.09(\mathrm{~d}, J=5.1 \mathrm{~Hz}$, 1H), $2.01-1.86$ (m, 1H); MS (ESI): $\mathrm{m} / z 392.3\left(\mathrm{M}^{+}+\mathrm{H}\right)$.

\section{Synthesis of Primary Amine 47}

Benzyl (R)-(3-hydroxy-1-(4-methoxyphenyl)propyl)carbamate (65). Benzyl chloroformate chloride $(226 \mu \mathrm{L}, 1.584 \mathrm{mmol})$ was added to a solution of $64(261 \mathrm{mg}$, $1.440 \mathrm{mmol})$ and trimethylamine $(0.504 \mathrm{~mL}, 3.60 \mathrm{mmol})$ in THF $(7 \mathrm{~mL})$. The reaction mixture was stirred for $14 \mathrm{~h}$ at room temperature. The reaction was concentrated and extracted with ethyl acetate and water. The organic phase was washed with brine, dried 
$\left(\mathrm{Na}_{2} \mathrm{SO}_{4}\right)$ and evaporated in vacuo. The residue was purified by flash column chromatography (Isolera 1, Biotage, $10 \mathrm{~g}$ size, elution gradient 12-60\% ethyl acetate in hexanes), to afford $\mathbf{6 5}$ as a white solid.

Benzyl (R)-(3-(1,3-dioxoisoindolin-2-yl)-1-(4-methoxyphenyl)propyl)carbamate (66). DIAD (152 $\mathrm{mL}, 0.785 \mathrm{mmol})$ was added dropwise at room temperature, to a solution of $65(0.225 \mathrm{~g}, 0.7135 \mathrm{mmol})$, phthalimide $(0.115 \mathrm{~g}, 0.785 \mathrm{mmol})$ and $\mathrm{Ph}_{3} \mathrm{P}$ $(0.206 \mathrm{~g}, 0.785 \mathrm{mmol})$ in THF $(3.5 \mathrm{~mL})$. The resulting mixture was stirred for $16 \mathrm{~h}$. The mixture was extracted with ethyl acetate/water and the organic phase was washed with brine, dried $\left(\mathrm{Na}_{2} \mathrm{SO}_{4}\right)$ and evaporated in vacuo. The residue was purified by flash column chromatography (Isolera 1, Biotage, $10 \mathrm{~g}$ size, elution gradient 12-60 ethyl acetate in hexanes), to afford 66 as a yellow solid.

Benzyl (R)-(3-amino-1-(4-methoxyphenyl)propyl)carbamate (67). Compound 66 $(0.317 \mathrm{~g}, 0.713 \mathrm{mmol})$ was dissolved in methanol and a $80 \%$ hydrazine hydrate solution $(0.109 \mathrm{~mL}, 1.783 \mathrm{mmol})$ was added dropwise to the solution at $0^{\circ} \mathrm{C}$. The reaction mixture was stirred for $16 \mathrm{~h}$ at room temperature. The mixture was subjected to acid-base extraction with ethyl acetate/water and the organic phase was washed with brine, dried $\left(\mathrm{Na}_{2} \mathrm{SO}_{4}\right)$ and concentrated in vacuo. The resulting residue 67 (colorless oil) was used in the next reaction without further purification.

Benzyl (R)-(3-(((l1-methyl)(l1-oxidaneyl)boraneyl)amino)-1-(4methoxyphenyl)propyl)carbamate (68). Di-tert-butyl dicarbonate $(0.143 \mathrm{~g}, 0.657 \mathrm{mmol})$ was added to a solution of $67(0.172 \mathrm{~g}, 0.547 \mathrm{mmol})$ and trimethylamine $(0.153 \mathrm{~mL}$, $1.094 \mathrm{mmol})$ in DCM (3 mL). The reaction mixture was stirred for $2 \mathrm{~h}$ at room temperature. The mixture was extracted with ethyl acetate/water and the organic phase was washed with brine, dried $\left(\mathrm{Na}_{2} \mathrm{SO}_{4}\right)$ and concentrated in vacuo. The residue $\mathbf{6 8}$ was used in the next step without further purification.

(R)-N1-((l1-methyl)(l1-oxidaneyl)boraneyl)-3-(4-methoxyphenyl)propane-1,3diamine (69). $\mathrm{Pd} / \mathrm{C}(0.200 \mathrm{~g}, 10 \% \mathrm{wet})$ was added to a solution of $\mathbf{6 8}(0.137 \mathrm{~g}, 0.331$ $\mathrm{mmol})$ in methanol $(8 \mathrm{~mL})$. The reaction was stirred under hydrogen for $3 \mathrm{~h}$ at room temperature. The mixture was filtered, and the filtrate was concentrated in vacuo. The residue 69 was used in the next step without further purification.

(R)-N3-((l1-methyl)(l1-oxidaneyl)boraneyl)-1-(4-methoxyphenyl)-N1-(6phenylthieno[2,3-d]pyrimidin-4-yl)propane-1,3-diamine (70). 4-chloro-6-phenylthienopyrimidine $7(0.45 \mathrm{~g}, 0.168 \mathrm{mmol})$, amine 69 (0.61 g, $0.218 \mathrm{mmol})$, and triethylamine $(0.36 \mathrm{~mL}, 0.254 \mathrm{mmol})$ were dissolved in ethanol and the mixture was heated at $115^{\circ} \mathrm{C}$ for $14 \mathrm{~h}$. The mixture was extracted with ethyl acetate/water and the organic phase was washed with brine, dried $\left(\mathrm{Na}_{2} \mathrm{SO}_{4}\right)$ and concentrated in vacuo. The residue was purified by column chromatography (Isolera 1, Biotage, $10 \mathrm{~g}$ size, elution gradient of $12-60 \%$ ethyl acetate in hexanes) to afford $\mathbf{7 0}$ as a yellow solid.

(R)-1-(4-methoxyphenyl)-N1-(6-phenylthieno[2,3-d]pyrimidin-4-yl)propane-1,3diamine (47). $\mathrm{HCl}$ (4M in 1,4-dioxane, $0.073 \mathrm{~mL}, 0.292 \mathrm{mmol}$ ) was added to a solution 
of $70(0.053 \mathrm{~g}, 0.215 \mathrm{mmol})$ in a mixture of DCM $(1 \mathrm{~mL})$ and methanol $(0.4 \mathrm{~mL})$, and the resulting mixture was stirred for $16 \mathrm{~h}$ at room temperature. The mixture was extracted with ethyl acetate/water and the organic phase was washed with brine, dried $\left(\mathrm{Na}_{2} \mathrm{SO}_{4}\right)$ and evaporated in vacuo to afford $\mathbf{4 7}$ as a yellow solid.

(R)-1-(4-methoxyphenyl)-N1-(6-phenylthieno[2,3-d]pyrimidin-4-yl)propane-1,3diamine (47). ${ }^{1} \mathrm{H}$ NMR (DMSO, $\left.500 \mathrm{MHZ}\right): \delta 8.41(\mathrm{~s}, 1 \mathrm{H}), 8.32(\mathrm{~s}, 1 \mathrm{H}), 8.11(\mathrm{~s}, 1 \mathrm{H})$, $7.73(\mathrm{~d}, 2 \mathrm{H}, \mathrm{J}=8.1 \mathrm{~Hz}), 7.53(\mathrm{t}, 2 \mathrm{H}, \mathrm{J}=7.45 \mathrm{~Hz}), 7.45-7.39(\mathrm{~m}, 2 \mathrm{H}), 6.93(\mathrm{~d}, 2 \mathrm{H}, \mathrm{J}=$ 8.75 Hz), 5.45-5.43 (m, 1H), 4.79-4.76 (m, 2H), 3.73 (s, 3H), 2.97-2.89 (m, 1H), 2.792.75 (m, 1H), 1.21-1.18 (m, 2H); MS (ESI): $m / z 391\left(\mathrm{M}^{+}+\mathrm{H}\right)$.

\section{Synthesis of Amine 48}

Tert-butyl (R)-(3-hydroxy-1-(4-methoxyphenyl)propyl)carbamate (71). Di-tertbutyl dicarbonate $(1.98 \mathrm{~g}, 9.10 \mathrm{mmol})$ was added to a solution of $64(1.5 \mathrm{~g}, 8.28 \mathrm{mmo})$ and trimethylamine $(1.27 \mathrm{~mL}, 9.10 \mathrm{mmol})$ in DCM $(40 \mathrm{~mL})$. The reaction mixture was stirred for $2 \mathrm{~h}$ a room temperature. The mixture was concentrated and purified by flash column chromatography (Isolera 1, Biotage, $10 \mathrm{~g}$ size, elution gradient $12-80 \%$ ethyl acetate in hexanes) to afford $\mathbf{7 1}$ as a white solid.

\section{(R)-3-((tert-butoxycarbonyl)amino)-3-(4-methoxyphenyl)propyl}

methanesulfonate (72). Methanesulfonyl chloride $(0.825 \mathrm{~mL}, 10.66 \mathrm{mmol})$ was added to a solution of $71(1 \mathrm{~g}, 3.55 \mathrm{mmol})$ and trimethylamine $(1.487 \mathrm{~mL}, 10.66 \mathrm{mmol})$ at $0^{\circ} \mathrm{C}$. The reaction mixture was stirred for $3 \mathrm{~h}$ at room temperature. The mixture was concentrated and purified by flash column chromatography (Isolera 1, Biotage, $10 \mathrm{~g}$ size, elution gradient $12-80 \%$ ethyl acetate in hexanes) to afford 72 as a yellow solid.

Tert-butyl (R)-(3-(dimethylamino)-1-(4-methoxyphenyl)propyl)carbamate (73). In a microwave tube, compound $72(0.075 \mathrm{~g}, 0.21 \mathrm{mmol})$ and tetrabutylammonium $(0.01$ $\mathrm{g}, 0.042 \mathrm{mmol}$ ) were dissolved with dimethylamine (2M in THF, $2 \mathrm{~mL}$ ) and subjected to microwave heating at $150^{\circ} \mathrm{C}$ for $0.5 \mathrm{~h}$. The mixture was extracted with ethyl acetate/water and the organic phase was washed with brine, dried $\left(\mathrm{Na}_{2} \mathrm{SO}_{4}\right)$ and concentrate in vacuo. The resulting residue $\mathbf{7 3}$ (bright red oil) was used in the next step without further purification.

(R)-1-(4-methoxyphenyl)-N3,N3-dimethylpropane-1,3-diamine (74). $\mathrm{HCl}$ (4 M in 1,4-dioxane, $0.422 \mathrm{~mL}, 1.688 \mathrm{mmol})$ was added to a solution of $73(0.13 \mathrm{~g}, 0.422$ $\mathrm{mmol})$ in a mixture of DCM $(2 \mathrm{~mL})$ and methanol $(0.8 \mathrm{~mL})$, and the resulting mixture was stirred for $16 \mathrm{~h}$ at room temperature. The mixture was subjected to acid-base extraction with ethyl acetate/water and the organic phase was washed with brine, dried $\left(\mathrm{Na}_{2} \mathrm{SO}_{4}\right)$ and concentrated in vacuo. The resulting residue 74 (colorless oil) was used in the next step without further purification.

(R)-1-(4-methoxyphenyl)-N3,N3-dimethyl-N1-(6-phenylthieno[2,3-d]pyrimidin4-yl)propane-1,3-diamine (48). 4-Chloro-6-phenyl-thienopyrimidine 7 (0.048 g, 0.195 
$\mathrm{mmol})$, amine $74(0.05 \mathrm{~g}, 0.204 \mathrm{mmol})$, and triethylamine $(0.056 \mathrm{~mL}, 0.408 \mathrm{mmol})$ were dissolved in ethanol and the mixture was heated at $115^{\circ} \mathrm{C}$ for $14 \mathrm{~h}$. The mixture was extracted with ethyl acetate/water and the organic phase was washed with brine, dried $\left(\mathrm{Na}_{2} \mathrm{SO}_{4}\right)$ and concentrated in vacuo. The residue was purified by column chromatography (Isolera 1, Biotage, $10 \mathrm{~g}$ size, elution gradient of 0-20\% methanol in ethyl acetate) to afford $\mathbf{4 8}$ as yellow solid.

(R)1-(4-methoxyphenyl)-N3,N3-dimethyl-N1-(6-phenylthieno[2,3-d]pyrimidin4-yl)propane-1,3-diamine (48). ${ }^{1} \mathrm{H}$ NMR (MeOD, $\left.500 \mathrm{MHz}\right) \delta 8.27(\mathrm{~d}, J=1.1 \mathrm{~Hz}, 1 \mathrm{H})$, $7.90(\mathrm{~s}, 1 \mathrm{H}), 7.70(\mathrm{dt}, J=8.4,1.7 \mathrm{~Hz}, 2 \mathrm{H}), 7.45(\mathrm{dd}, J=8.5,7.1 \mathrm{~Hz}, 2 \mathrm{H}), 7.41-7.34$ (m, $3 \mathrm{H}), 6.92-6.88(\mathrm{~m}, 2 \mathrm{H}), 5.44(\mathrm{t}, J=7.3 \mathrm{~Hz}, 1 \mathrm{H}), 3.80-3.75(\mathrm{~m}, 3 \mathrm{H}), 2.48(\mathrm{ddd}, J=$ 12.6, 9.9, $5.3 \mathrm{~Hz}, 1 \mathrm{H}), 2.37(\mathrm{ddd}, J=12.6,9.9,6.0 \mathrm{~Hz}, 1 \mathrm{H}), 2.28(\mathrm{~s}, 6 \mathrm{H}), 2.21-2.09$ (m, 2H); MS (ESI): $m / z 419\left(\mathrm{M}^{+}+\mathrm{H}\right)$.

\section{Synthesis of Thienopyrimidine Analog 49}

49 was synthesized following the general procedure for the amination 4-chloro-6phenylthieno[2,3- $d]$ pyrimidine.

(R)-3-((6-phenylthieno[2,3-d]pyrimidin-4-yl)amino)-3-(pyridin-4-yl)propan-1-ol (49). ${ }^{1} \mathrm{H}$ NMR (MeOD, $\left.500 \mathrm{MHz}\right) \delta 8.54-8.44$ (m, 2H), 8.26 (d, $\left.J=2.7 \mathrm{~Hz}, 1 \mathrm{H}\right), 7.96$ (s, 1H), $7.81-7.70(\mathrm{~m}, 2 \mathrm{H}), 7.59-7.51(\mathrm{~m}, 2 \mathrm{H}), 7.49$ (dd, $J=8.5,7.0 \mathrm{~Hz}, 2 \mathrm{H}), 7.44-$ $7.37(\mathrm{~m}, 1 \mathrm{H}), 5.65(\mathrm{dd}, J=9.2,5.5 \mathrm{~Hz}, 1 \mathrm{H}), 3.81-3.66(\mathrm{~m}, 2 \mathrm{H}), 2.21$ (qdt, $J=13.9,7.8$, $5.5 \mathrm{~Hz}, 2 \mathrm{H})$; MS (ESI): $m / z 363\left(\mathrm{M}^{+}+\mathrm{H}\right)$.

\section{Synthesis of Thienopyrimidine and Pyrrolopyrimidine Analogs with Modifications at the 6-position}

2-((6-bromothieno[2,3-d]pyrimidin-4-yl)amino)-2-(4-methoxyphenyl)ethan-1-ol (76a). 4-Chloro-6-bromo-thienopyrimdine 75a (0.025 mg, $0.100 \mathrm{mmol})$, amine 59 (0.050 $\mathrm{mg}, 0.299 \mathrm{mmol})$ and trimethylamine $(0.042 \mathrm{~mL}, 0.299 \mathrm{mmol})$ were dissolved in ethanol and the mixture was heated at $115{ }^{\circ} \mathrm{C}$ for $14 \mathrm{~h}$. The mixture was extracted with ethyl acetate/water and the organic phase was washed with brine, dried $\left(\mathrm{Na}_{2} \mathrm{SO}_{4}\right)$ and concentrated in vacuo. The resulting residue 76a (red solid, with the pyrrolopyrimidine) was used in the next reaction without further purification.

\section{2-(4-methoxyphenyl)-2-((6-(pyridin-4-yl)thieno[2,3-d]pyrimidin-4-}

yl)amino)ethan-1-ol (50). Compound 76a (0.08 g, $0.210 \mathrm{mmol})$, tetraphenyl pallidium $(0.005 \mathrm{~g}, 0.0012 \mathrm{mmol})$ and compound 77a $(0.039 \mathrm{~g}, 0.316 \mathrm{mmol})$ were dissolved in dioxane $(4 \mathrm{~mL})$. The solution was bubbled with nitrogen for $15 \mathrm{~min}$. Then, an aqueous solution of $\mathrm{NaHCO}_{3}(0.083 \mathrm{~g}, 0.993 \mathrm{mmol})$ was added and the mixture was heated at 75 ${ }^{\circ} \mathrm{C}$ for $14 \mathrm{~h}$. The mixture was extracted with ethyl acetate/water and the organic phase was washed with brine, dried $\left(\mathrm{Na}_{2} \mathrm{SO}_{4}\right)$ and concentrated in vacuo. The residue was purified 
by column chromatography (Isolera 1, Biotage, $10 \mathrm{~g}$ size, elution gradient of $0-20 \%$ methanol in ethyl acetate) to afford $\mathbf{5 0}$ as yellow solid.

2-(4-methoxyphenyl)-2-((6-(pyridin-4-yl)thieno[2,3-d]pyrimidin-4yl)amino)ethan-1-ol (50). ${ }^{1} \mathrm{H}$ NMR (MeOD, $\left.400 \mathrm{MHz}\right) \delta 8.31-8.18(\mathrm{~m}, 1 \mathrm{H}), 8.03-$ $7.93(\mathrm{~m}, 1 \mathrm{H}), 7.81-7.68(\mathrm{~m}, 2 \mathrm{H}), 7.52-7.45(\mathrm{~m}, 2 \mathrm{H}), 7.43-7.36(\mathrm{~m}, 3 \mathrm{H}), 6.92$ (ddd, $J$ $=8.5,3.2,1.5 \mathrm{~Hz}, 2 \mathrm{H}), 5.52(\mathrm{~s}, 1 \mathrm{H}), 3.94(\mathrm{dd}, J=5.8,3.5 \mathrm{~Hz}, 2 \mathrm{H}), 3.82-3.70(\mathrm{~m}, 3 \mathrm{H})$; MS (ESI): $m / z 379.4\left(\mathrm{M}^{+}+\mathrm{H}\right)$.

\section{2-(4-methoxyphenyl)-2-((6-(pyridin-3-yl)thieno[2,3-d]pyrimidin-4-} yl)amino)ethan-1-ol (51). Compound 76a (0.1 g, $0.263 \mathrm{mmol})$, tetraphenyl pallidium (0.005 g, $0.0012 \mathrm{mmol})$ and compound 77b (0.048 g, $0.394 \mathrm{mmol})$ were dissolved in dioxane $(4 \mathrm{~mL})$. The solution was bubbled with nitrogen for $15 \mathrm{~min}$. Then, an aqueous solution of $\mathrm{NaHCO}_{3}(0.104 \mathrm{~g}, 1.238 \mathrm{mmol})$ was added and the mixture was heated at 75 ${ }^{\circ} \mathrm{C}$ for $14 \mathrm{~h}$. The mixture was extracted with ethyl acetate/water and the organic phase was washed with brine, dried $\left(\mathrm{Na}_{2} \mathrm{SO}_{4}\right)$ and concentrated in vacuo. The residue was purified by column chromatography (Isolera 1, Biotage, $10 \mathrm{~g}$ size, elution gradient of $0-20 \%$ methanol in ethyl acetate) to afford $\mathbf{5 1}$ as yellow solid.

\section{2-(4-methoxyphenyl)-2-((6-(pyridin-3-yl)thieno[2,3-d]pyrimidin-4-} yl)amino)ethan-1-ol (51). ${ }^{1} \mathrm{H}$ NMR (DMSO, $\left.500 \mathrm{MHz}\right) \delta 8.95$ (d, $\left.J=2.5 \mathrm{~Hz}, 1 \mathrm{H}\right), 8.60$ $(\mathrm{dd}, J=4.8,1.5 \mathrm{~Hz}, 1 \mathrm{H}), 8.31(\mathrm{~s}, 1 \mathrm{H}), 8.35(\mathrm{~s}, 1 \mathrm{H}), 8.23(\mathrm{~d}, J=8.0 \mathrm{~Hz}, 1 \mathrm{H}), 8.08$ (dt, $J=$ 8.0, $2.0 \mathrm{~Hz}, 1 \mathrm{H}), 7.40-7.32(\mathrm{~m}, 2 \mathrm{H}), 6.93-6.86(\mathrm{~m}, 2 \mathrm{H}), 5.41(\mathrm{td}, J=7.9,5.3 \mathrm{~Hz}, 1 \mathrm{H})$, 5.04 (s, 2H), 3.72 (s, 4H); MS (ESI): $m / z 379.2\left(\mathrm{M}^{+}+\mathrm{H}\right)$.

\section{2-(4-methoxyphenyl)-2-((6-(pyridin-2-yl)thieno[2,3-d]pyrimidin-4-}

yl)amino)ethan-1-ol (52). Compound 76a (0.1 g, $0.263 \mathrm{mmol})$, tetraphenyl pallidium (0.005 g, $0.0012 \mathrm{mmol})$ and compound 77c (0.048 g, $0.394 \mathrm{mmol})$ were dissolved in dioxane $(4 \mathrm{~mL})$. The solution was bubbled with nitrogen for $15 \mathrm{~min}$. Then, an aqueous solution of $\mathrm{NaHCO}_{3}(0.104 \mathrm{~g}, 1.238 \mathrm{mmol})$ was added and the mixture was heated at 75 ${ }^{\circ} \mathrm{C}$ for $14 \mathrm{~h}$. The mixture was extracted with ethyl acetate/water and the organic phase was washed with brine, dried $\left(\mathrm{Na}_{2} \mathrm{SO}_{4}\right)$ and concentrated in vacuo. The residue was purified by column chromatography (Isolera 1, Biotage, $10 \mathrm{~g}$ size, elution gradient of $0-20 \%$ methanol in ethyl acetate) to afford $\mathbf{5 2}$ as yellow solid.

\section{2-(4-methoxyphenyl)-2-((6-(pyridin-2-yl)thieno[2,3-d]pyrimidin-4-} yl)amino)ethan-1-ol (52). ${ }^{1} \mathrm{H}$ NMR (DMSO, $\left.400 \mathrm{MHz}\right) \delta 8.95$ (d, J=2.4 Hz, 1H), 8.60 $(\mathrm{dd}, J=4.8,1.5 \mathrm{~Hz}, 1 \mathrm{H}), 8.33$ (d, $J=12.6 \mathrm{~Hz}, 2 \mathrm{H}), 8.20$ (d, $J=8.0 \mathrm{~Hz}, 1 \mathrm{H}), 8.08$ (ddd, $J$ $=8.0,2.5,1.5 \mathrm{~Hz}, 1 \mathrm{H}), 7.36(\mathrm{~d}, J=8.6 \mathrm{~Hz}, 2 \mathrm{H}), 6.92-6.87(\mathrm{~m}, 2 \mathrm{H}), 5.41(\mathrm{q}, J=7.5 \mathrm{~Hz}$, $1 \mathrm{H}), 5.02(\mathrm{t}, J=5.7 \mathrm{~Hz}, 1 \mathrm{H}), 3.72(\mathrm{~s}, 5 \mathrm{H}) ; \mathrm{MS}(\mathrm{ESI}): \mathrm{m} / z 379.4\left(\mathrm{M}^{+}+\mathrm{H}\right)$.

\section{2-((6-(1H-pyrazol-4-yl)thieno[2,3-d]pyrimidin-4-yl)amino)-2-(4-} methoxyphenyl)ethan-1-ol (53). Compound 76a (0.1 g, $0.263 \mathrm{mmol})$, tetraphenyl pallidium $(0.005 \mathrm{~g}, 0.0012 \mathrm{mmol})$ and compound $77 \mathbf{d}(0.044 \mathrm{~g}, 0.394 \mathrm{mmol})$ were dissolved in dioxane $(4 \mathrm{~mL})$. The solution was bubbled with nitrogen for $15 \mathrm{~min}$. Then, an aqueous solution of $\mathrm{NaHCO}_{3}(0.104 \mathrm{~g}, 1.238 \mathrm{mmol})$ was added and the mixture was 
heated at $75{ }^{\circ} \mathrm{C}$ for $14 \mathrm{~h}$. The mixture was extracted with ethyl acetate/water and the organic phase was washed with brine, dried $\left(\mathrm{Na}_{2} \mathrm{SO}_{4}\right)$ and concentrated in vacuo. The residue was purified by column chromatography (Isolera 1, Biotage, $10 \mathrm{~g}$ size, elution gradient of $0-20 \%$ methanol in ethyl acetate) to afford $\mathbf{5 3}$ as yellow solid.

\section{2-((6-(1H-pyrazol-4-yl)thieno[2,3-d]pyrimidin-4-yl)amino)-2-(4-} methoxyphenyl)ethan-1-ol (53). ${ }^{1} \mathrm{H}$ NMR (DMSO, $\left.400 \mathrm{MHz}\right) \delta 8.22(\mathrm{~d}, J=1.6 \mathrm{~Hz}, 1 \mathrm{H})$, $8.08(\mathrm{~d}, J=7.9 \mathrm{~Hz}, 1 \mathrm{H}), 7.84(\mathrm{~s}, 1 \mathrm{H}), 7.39-7.30(\mathrm{~m}, 2 \mathrm{H}), 6.92-6.83(\mathrm{~m}, 2 \mathrm{H}), 5.36(\mathrm{q}, J$ $=7.8 \mathrm{~Hz}, 1 \mathrm{H}), 5.07(\mathrm{~s}, 1 \mathrm{H}), 3.72(\mathrm{~d}, J=1.4 \mathrm{~Hz}, 5 \mathrm{H}) ; \mathrm{MS}(\mathrm{ESI}): \mathrm{m} / z 368.3\left(\mathrm{M}^{+}+\mathrm{H}\right)$.

\section{Synthesis of Thienopyrimidine Analog 54}

54 was synthesized following the general procedure for the amination 4-chloro-6phenylthieno[2,3-d]pyrimidine.

2-((5,6-dimethylthieno[2,3-d]pyrimidin-4-yl)amino)-2-(4-methoxyphenyl)ethan1-ol (54). ${ }^{1} \mathrm{H}$ NMR $\left(\mathrm{CDCl}_{3}, 400 \mathrm{MHz}\right) \delta 8.33$ (s, 1H), 7.35 - 7.28 (m, 2H), 6.92 (dd, $J=$ $8.4,1.3 \mathrm{~Hz}, 2 \mathrm{H}), 6.15(\mathrm{~d}, J=5.8 \mathrm{~Hz}, 1 \mathrm{H}), 5.35(\mathrm{q}, J=5.5 \mathrm{~Hz}, 1 \mathrm{H}), 4.06-3.96(\mathrm{~m}, 2 \mathrm{H})$, 3.81 (d, $J=1.0 \mathrm{~Hz}, 3 \mathrm{H}), 2.49-2.38(\mathrm{~m}, 6 \mathrm{H})$. MS (ESI): $\mathrm{m} / z 330.01\left(\mathrm{M}^{+}+\mathrm{H}\right)$.

\section{Synthesis of Fluorophenyl Thienopyrimidine Analog 55}

Ethyl 2-amino-5-(4-fluorophenyl)thiophene-3-carboxylate (79). In a microwave tube, ethyl 3-cyanoacetate $4(0.693 \mathrm{~mL}, 6.52 \mathrm{mmol})$ was added to a solution of 2-(4fluorophenyl)acetaldehyde $78(0.804 \mathrm{~g}, 6.52 \mathrm{mmol})$, sulfur $(0.23 \mathrm{~g}, 7.17 \mathrm{mmol})$ and morpholine $(0.651 \mathrm{~mL}, 7.54 \mathrm{mmol})$ in ethanol $(20 \mathrm{~mL})$. The resulting mixture was submitted to microwave heating for $30 \mathrm{~min}$ at $70^{\circ} \mathrm{C}$. After cooling, the mixture was filtered, and the filtrate was poured in water. The precipitate was collected, dried and purified by flash column chromatography (Isolera 1, Biotage, $25 \mathrm{~g}$ size, elution gradient $12-60 \%$ ethyl acetate in hexanes) to afford $\mathbf{7 9}$ as a yellow solid.

Ethyl 2-amino-5-(4-fluorophenyl)thiophene-3-carboxylate (79). ${ }^{1} \mathrm{H}$ NMR (MeOD, $500 \mathrm{MHZ}): \delta$ 7.45-7.41 (m, 2H), $7.14(\mathrm{~s}, 1 \mathrm{H}), 7.08-7.03(\mathrm{~m}, 2 \mathrm{H}), 4.58(\mathrm{~s}, 2 \mathrm{H})$, 4.30-4.24 (q, 2H, $J=9.05 \mathrm{~Hz}), 1.37-1.29$ (t, $3 \mathrm{H}, J=8.95 \mathrm{~Hz}$ ); MS (ESI): $\mathrm{m} / z 266$ $\left(\mathrm{M}^{+}+\mathrm{H}\right)$.

6-(4-fluorophenyl)thieno[2,3-d]pyrimidin-4(3H)-one (80). Compound 79 (1.15 $\mathrm{g}, 4.33 \mathrm{mmol}$ ) was dissolved in excess formamide and the resulting mixture was heated for $16 \mathrm{~h}$ at $160^{\circ} \mathrm{C}$. The mixture was concentrated in vacuo and purified by flash column chromatography (Isolera 1, Biotage, $25 \mathrm{~g}$ size, elution gradient 12-60\% ethyl acetate in hexanes) to afford $\mathbf{8 0}$ as a red solid.

6-(4-fluorophenyl)thieno[2,3-d]pyrimidin-4(3H)-one (80). ${ }^{1} \mathrm{H}$ NMR (DMSO, 500 MHZ): $\delta 8.41$ (s, 1H), 7.85-7.81 (m, 2H), 7.78 (s, 1H), 7.33-7.27 (m, 2H); MS (ESI): $m / z 247\left(\mathrm{M}^{+}+\mathrm{H}\right)$. 
4-chloro-6-(4-fluorophenyl)thieno[2,3-d]pyrimidine (81). Compound 80 (0.32 g, $0.13 \mathrm{mmol})$ and $\mathrm{N}, \mathrm{N}$-dimethylaniline $(0.001 \mathrm{~mL}, 0.009 \mathrm{mmol})$ were dissolved in phosphoryl trichloride $(0.5 \mathrm{~mL}, 8.12 \mathrm{mmol})$ and the mixture was heated for $16 \mathrm{~h}$ to $95^{\circ} \mathrm{C}$. The mixture was concentrated in vacuo and purified by flash column chromatography (Isolera 1, Biotage, $25 \mathrm{~g}$ size, elution gradient of 12-60\% ethyl acetate in hexanes) to afford $\mathbf{8 1}$ as a yellow solid.

4-chloro-6-(4-fluorophenyl)thieno[2,3-d]pyrimidine (81). ${ }^{1} \mathrm{H} \mathrm{NMR}\left(\mathrm{CDCl}_{3}, 500\right.$ MHZ): $\delta 8.82(\mathrm{~s}, 1 \mathrm{H}), 7.74-7.69(\mathrm{~m}, 2 \mathrm{H}), 7.52(\mathrm{~s}, 1 \mathrm{H}), 7.22-7.16(\mathrm{~m}, 2 \mathrm{H}) ; \mathrm{MS}(\mathrm{ESI}): \mathrm{m} / z$ $265\left(\mathrm{M}^{+}+\mathrm{H}\right)$.

In the last step, $\mathbf{5 5}$ was synthesized following the general procedure for the amination 4-chloro-6-phenylthieno[2,3- $d]$ pyrimidine.

\section{2-((6-(4-fluorophenyl)thieno[2,3-d]pyrimidin-4-yl)amino)-2-(4-} methoxyphenyl)ethan-1-ol (55). ${ }^{1} \mathrm{H} \mathrm{NMR}\left(\mathrm{CDCl}_{3}, 400 \mathrm{MHz}\right) \delta 8.45(\mathrm{~s}, 1 \mathrm{H}), 7.66-7.59$ $(\mathrm{m}, 2 \mathrm{H}), 7.35(\mathrm{~d}, J=8.7 \mathrm{~Hz}, 2 \mathrm{H}), 7.13(\mathrm{t}, J=8.6 \mathrm{~Hz}, 3 \mathrm{H}), 6.94(\mathrm{~d}, J=8.7 \mathrm{~Hz}, 2 \mathrm{H}), 5.72$ $(\mathrm{d}, J=6.2 \mathrm{~Hz}, 1 \mathrm{H}), 5.40(\mathrm{~d}, J=4.0 \mathrm{~Hz}, 1 \mathrm{H}), 4.13-4.03(\mathrm{~m}, 3 \mathrm{H}), 3.82$ (s, 3H); MS (ESI): $m / z 396.2\left(\mathrm{M}^{+}+\mathrm{H}\right)$.

Synthesis of Pyrrolopyrimidine and Quinazoline Analogs with Modification at the 6position

2-((6-bromo-7H-pyrrolo[2,3-d]pyrimidin-4-yl)amino)-2-(4methoxyphenyl)ethan-1-ol (76b). 6-Bromo-4-chloro-7H-pyrrolo[2,3- $d]$ pyrimdine $75 \mathrm{~b}$ $(0.070 \mathrm{mg}, 0.301 \mathrm{mmol})$, amine $59(0.076 \mathrm{mg}, 0.452 \mathrm{mmol})$ and trimethylamine $(0.126$ $\mathrm{mL}, 0.904 \mathrm{mmol}$ ) were dissolved in ethanol and the mixture was heated at $115{ }^{\circ} \mathrm{C}$ for 14h. The mixture was extracted with ethyl acetate/water and the organic phase was washed with brine, dried $\left(\mathrm{Na}_{2} \mathrm{SO}_{4}\right)$ and concentrated in vacuo. The resulting residue $\mathbf{7 6} \mathbf{b}$ was used in the next reaction without further purification.

\section{2-(4-methoxyphenyl)-2-((6-phenyl-7H-pyrrolo[2,3-d]pyrimidin-4-}

yl)amino)ethan-1-ol (56). Compound 76b (0.081 g, $0.263 \mathrm{mmol})$, tetraphenyl pallidium $(0.005 \mathrm{~g}, 0.0012 \mathrm{mmol})$ and compound $77 \mathrm{e}(0.039 \mathrm{~g}, 0.316 \mathrm{mmol})$ were dissolved in dioxane $(4 \mathrm{~mL})$. The solution was bubbled with nitrogen for $15 \mathrm{~min}$. Then, an aqueous solution of $\mathrm{NaHCO}_{3}(0.083 \mathrm{~g}, 0.993 \mathrm{mmol})$ was added and the mixture was heated at 75 ${ }^{\circ} \mathrm{C}$ for $14 \mathrm{~h}$. The mixture was extracted with ethyl acetate/water and the organic phase was washed with brine, dried $\left(\mathrm{Na}_{2} \mathrm{SO}_{4}\right)$ and concentrated in vacuo. The residue was purified by column chromatography (Isolera 1, Biotage, $10 \mathrm{~g}$ size, elution gradient of $0-20 \%$ methanol in ethyl acetate) to afford $\mathbf{5 6}$ as yellow solid.

\section{2-(4-methoxyphenyl)-2-((6-phenyl-7H-pyrrolo[2,3-d]pyrimidin-4-} yl)amino)ethan-1-ol (56). ${ }^{1} \mathrm{H}$ NMR (DMSO, $\left.500 \mathrm{MHz}\right) \delta 12.11(\mathrm{~s}, 1 \mathrm{H}), 8.08(\mathrm{~s}, 1 \mathrm{H})$, $7.80(\mathrm{~d}, J=7.8 \mathrm{~Hz}, 2 \mathrm{H}), 7.45(\mathrm{t}, J=7.6 \mathrm{~Hz}, 2 \mathrm{H}), 7.35(\mathrm{~d}, J=8.3 \mathrm{~Hz}, 2 \mathrm{H}), 7.30$ (t, $J=7.4$ 
$\mathrm{Hz}, 1 \mathrm{H}), 7.15(\mathrm{~s}, 1 \mathrm{H}), 6.91-6.83(\mathrm{~m}, 2 \mathrm{H}), 5.36$ (q, $J=7.4 \mathrm{~Hz}, 1 \mathrm{H}), 4.95(\mathrm{~s}, 1 \mathrm{H}), 3.81-$ 3.64 (m, 5H); MS (ESI): $m / z 361.4\left(\mathrm{M}^{+}+\mathrm{H}\right)$.

2-((7-bromoquinazolin-4-yl)amino)-2-(4-methoxyphenyl)ethan-1-ol (83). 7bromo-4-chloroquinazoline $82(0.2 \mathrm{~g}, 0.821 \mathrm{mmol})$, amine $59(0.208 \mathrm{~g}, 1.232 \mathrm{mmol})$ and trimethylamine $(0.573 \mathrm{~mL}, 4.11 \mathrm{mmol})$ were dissolved in ethanol and the mixture was subjected to microwave heating at $150{ }^{\circ} \mathrm{C}$ for $1 \mathrm{~h}$. The mixture was extracted with ethyl acetate/water and the organic phase was washed with brine, dried $\left(\mathrm{Na}_{2} \mathrm{SO}_{4}\right)$ and concentrated in vacuo. The resulting residue $\mathbf{8 3}$ was used in the next reaction without further purification.

\section{2-(4-methoxyphenyl)-2-((7-phenylquinazolin-4-yl)amino)ethan-1-ol (57).} Compound 83 (0.1 g, $0.267 \mathrm{mmol})$, tetraphenyl pallidium $(0.005 \mathrm{~g}, 0.0012 \mathrm{mmol})$ and phenylboronic acid 77e $(0.049 \mathrm{~g}, 0.401 \mathrm{mmol})$ were dissolved in dioxane $(4 \mathrm{~mL})$. The solution was bubbled with nitrogen for $15 \mathrm{~min}$. Then, an aqueous solution of $\mathrm{NaHCO}_{3}$ $(0.185 \mathrm{~g}, 1.336 \mathrm{mmol})$ was added and the mixture was heated at $75^{\circ} \mathrm{C}$ for $14 \mathrm{~h}$. The mixture was extracted with ethyl acetate/water and the organic phase was washed with brine, dried $\left(\mathrm{Na}_{2} \mathrm{SO}_{4}\right)$ and concentrated in vacuo. The residue was purified by column chromatography (Isolera 1, Biotage, $10 \mathrm{~g}$ size, elution gradient of $0-100 \%$ ethyl acetate in hexanes) to afford $\mathbf{5 7}$ as white solid.

2-(4-methoxyphenyl)-2-((7-phenylquinazolin-4-yl)amino)ethan-1-ol (57). ${ }^{1} \mathrm{H}$ NMR (DMSO, $500 \mathrm{MHz}) \delta 8.57(\mathrm{~d}, J=8.6 \mathrm{~Hz}, 1 \mathrm{H}), 8.44-8.40(\mathrm{~m}, 2 \mathrm{H}), 8.21(\mathrm{~s}, 1 \mathrm{H})$, $7.93-7.87(\mathrm{~m}, 2 \mathrm{H}), 7.87-7.83(\mathrm{~m}, 2 \mathrm{H}), 7.53(\mathrm{dd}, J=8.3,7.0 \mathrm{~Hz}, 2 \mathrm{H}), 7.48-7.43(\mathrm{~m}$, $1 \mathrm{H}), 7.40-7.34(\mathrm{~m}, 2 \mathrm{H}), 6.90-6.85(\mathrm{~m}, 2 \mathrm{H}), 5.48(\mathrm{td}, J=8.2,5.2 \mathrm{~Hz}, 1 \mathrm{H}), 3.84(\mathrm{dd}, J$ $=11.2,8.6 \mathrm{~Hz}, 1 \mathrm{H}), 3.71(\mathrm{~s}, 4 \mathrm{H}) . \mathrm{MS}(\mathrm{ESI}): m / z 372.4\left(\mathrm{M}^{+}+\mathrm{H}\right)$.

\section{Synthesis of Quinazoline Analog 58}

2-((6-bromoquinazolin-4-yl)amino)-2-(4-methoxyphenyl)ethan-1-ol (81). 6bromo-4-chloroquinazoline 75c $(0.200 \mathrm{~g}, 0.821 \mathrm{mmol})$, amine 59 (0.208 g, $1.232 \mathrm{mmol})$ and trimethylamine $(0.573 \mathrm{~mL}, 4.11 \mathrm{mmol})$ were dissolved in ethanol and the mixture was subjected to microwave heating at $150{ }^{\circ} \mathrm{C}$ for $1 \mathrm{~h}$. The mixture was extracted with ethyl acetate/water and the organic phase was washed with brine, dried $\left(\mathrm{Na}_{2} \mathrm{SO}_{4}\right)$ and concentrated in vacuo. The resulting residue 76c was used in the next reaction without further purification.

\section{2-(4-methoxyphenyl)-2-((6-phenylquinazolin-4-yl)amino)ethan-1-ol (58).} Compound 76c $(0.1 \mathrm{~g}, 0.267 \mathrm{mmol})$, tetraphenyl pallidium $(0.005 \mathrm{~g}, 0.0012 \mathrm{mmol})$ and phenylboronic acid 77e $(0.049 \mathrm{~g}, 0.401 \mathrm{mmol})$ were dissolved in dioxane $(4 \mathrm{~mL})$. The solution was bubbled with nitrogen for $15 \mathrm{~min}$. Then, an aqueous solution of $\mathrm{NaHCO}_{3}$ $(0.185 \mathrm{~g}, 1.336 \mathrm{mmol})$ was added and the mixture was heated at $75{ }^{\circ} \mathrm{C}$ for $14 \mathrm{~h}$. The mixture was extracted with ethyl acetate/water and the organic phase was washed with brine, dried $\left(\mathrm{Na}_{2} \mathrm{SO}_{4}\right)$ and concentrated in vacuo. The residue was purified by column 
chromatography (Isolera 1, Biotage, $10 \mathrm{~g}$ size, elution gradient of $0-100 \%$ ethyl acetate in hexanes) to afford $\mathbf{5 8}$ as yellow solid.

2-(4-methoxyphenyl)-2-((6-phenylquinazolin-4-yl)amino)ethan-1-ol (58). ${ }^{1} \mathrm{H}$ NMR (500 MHz, DMSO) $\delta 8.77(\mathrm{~d}, J=2.1 \mathrm{~Hz}, 1 \mathrm{H}), 8.53(\mathrm{~d}, J=7.9 \mathrm{~Hz}, 1 \mathrm{H}), 8.40$ (s, $1 \mathrm{H}), 8.15(\mathrm{~s}, 1 \mathrm{H}), 8.11(\mathrm{dd}, J=8.6,2.0 \mathrm{~Hz}, 1 \mathrm{H}), 7.92-7.82(\mathrm{~m}, 2 \mathrm{H}), 7.78-7.71(\mathrm{~m}$, $1 \mathrm{H}), 7.56(\mathrm{t}, J=7.8 \mathrm{~Hz}, 2 \mathrm{H}), 7.44(\mathrm{dd}, J=8.2,6.5 \mathrm{~Hz}, 1 \mathrm{H}), 7.41-7.32(\mathrm{~m}, 2 \mathrm{H}), 6.93-$ $6.82(\mathrm{~m}, 2 \mathrm{H}), 5.51(\mathrm{td}, J=8.2,5.2 \mathrm{~Hz}, 1 \mathrm{H}), 5.02(\mathrm{~s}, 1 \mathrm{H}), 3.85(\mathrm{dd}, J=11.2,8.6 \mathrm{~Hz}, 1 \mathrm{H})$, $3.81-3.61$ (m, 4H); MS (ESI): $m / z 372.3\left(\mathrm{M}^{+}+\mathrm{H}\right)$.

\section{Results and Discussion}

\section{Chemistry}

The synthesis of the amine compounds $\mathbf{4 6}$ and $\mathbf{3 1}$ (was carried out as described in Scheme 3-1. Starting from the amino-2-(4-methoxyphenyl)ethan-1-ol 59, Boc-protection of the free amine gave alcohol $\mathbf{6 0}$. Mitsunobu conditions were then used to introduce the targeted terminal amine as a phthalimide group to afford the intermediate 61. After Bocdeprotection with $\mathrm{HCl}, \mathbf{6 2}$ was reacted with 4-chloro, 6 phenyl thienopyrimidine $\mathbf{6 2}$ to give the substituted thienopyrimidine $\mathbf{6 3}$, deprotection of the phthalimide group in the presence of hydrazine afforded 46. The second targeted amine, compound 47, was synthesized using a similar strategy as described in Scheme 3-2. Starting from (R)-3amino-3-(4-methoxyphenyl)propanol 64, amine protection with the benzyl chloroformate afforded 65. Then, a phthalimide group was again introduced under mitsonubu conditions, to yield intermediate 66. Cbz-protection of the (R)-3-amino-3-(4methoxyphenyl)propanol 64 , from the previous step, prevents the attack of amine to the ketone of the phthalimide group that results in the cyclized side product. Deprotection of the phthalimide and subsequent protection of the free amine 67 with di-tert-butyl dicarbonate gave compound 68. Deprotection reaction of benzyl chloroformate $(\mathrm{Cbz})$ yielded amine 69, which was coupled to 4-chloro, 6-phenylthienopyrimidine 7 to afford 70. The targeted amine compound $\mathbf{4 7}$ was achieved following the final Boc-deprotection reaction of $\mathbf{7 0}$ in the presence of $\mathrm{HCl}$.

To examine the SAR of $\mathbf{4 7}$, the dimethyl amine analog of $\mathbf{4 7}, \mathbf{4 8}$, was generated Scheme 3-3. Starting from (R)-3-amino-3-(4-methoxyphenyl)propanol 64, Bocprotection of the free amine gave compound 71. Then, mesylation of alcohol group with mesylchloride afforded the intermediate 72. Displacement of the mesylate group with dimethylamine in THF gave tertiary amine intermediate 73. Acid catalyzed deprotection yielded the free amine 74, which was coupled to 4-chloro, 6-phenyl, thienopyrimidine to afford the targeted compound $\mathbf{4 8}$. To replace the para methoxy phenyl moiety of $\mathbf{2 5}$, with a less lipophilic, pyridinyl ring, compounds 49 was synthesized following the general procedure for the amination of 4-chloro, 6-phenyl, thienopyrimidine. Next synthetic efforts aimed to diminish the lipophilicity by substitution to phenyl ring at the 6 position of the thienopyrimidine. Pyridyl and pyrazolyl analogs were synthesized as described in 
Scheme 3-1. Synthesis of amine 46.

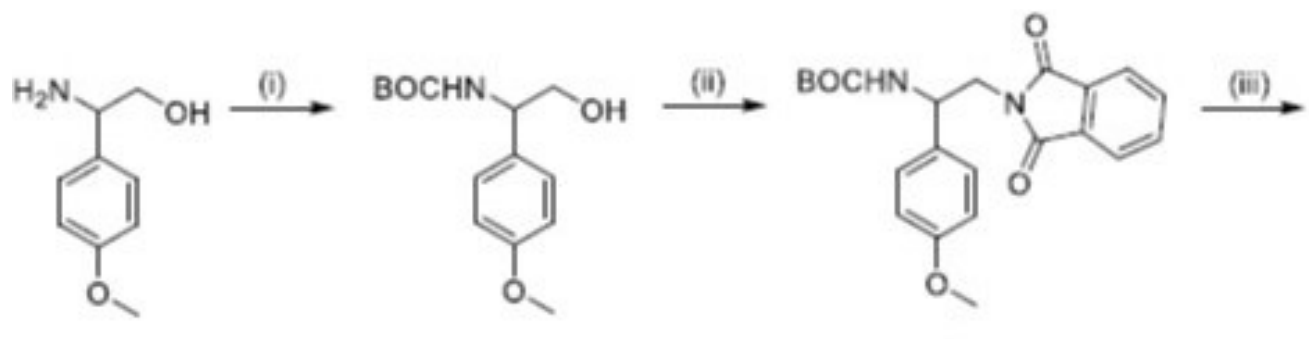

59

60

61
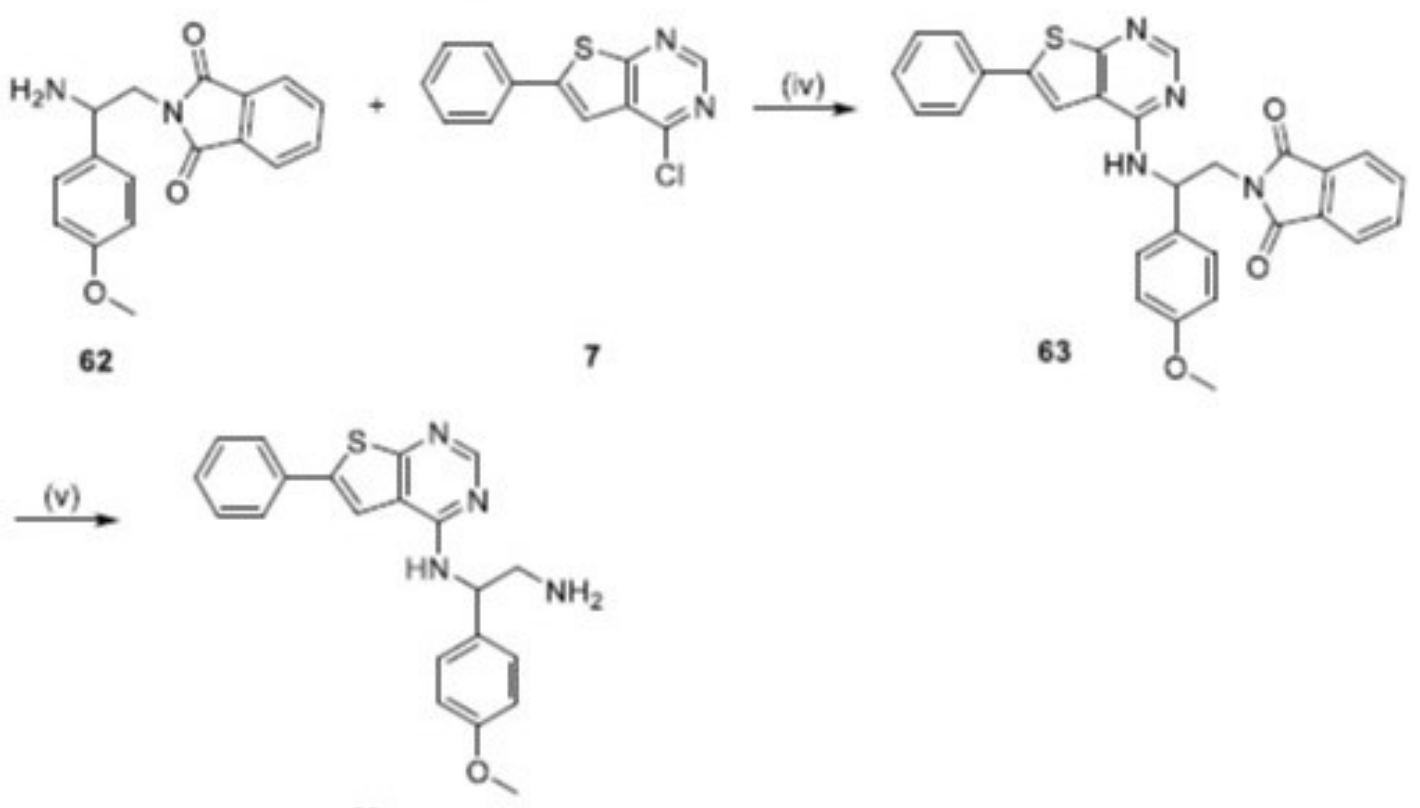

46

Reagents and Conditions: (i) di-tert-butyl decarbonate, Et ${ }_{3} \mathrm{~N}, \mathrm{DCM}, \mathrm{RT}, 2 \mathrm{~h}$; (ii)

Phthalimide, DIAD, $\mathrm{Ph}_{3} \mathrm{P}, \mathrm{DCM}, \mathrm{RT}$, 4h; (iii) $4 \mathrm{M} \mathrm{HCl}$ in dioxane, DCM, methanol, RT, 4 h; (iv) Et 3 N, reflux, 6 h; (v) $\mathrm{NH}_{2} \mathrm{NH}_{2}$, methanol, RT, 6 h. 
Scheme 3-2. Synthesis of the primary amine 47.
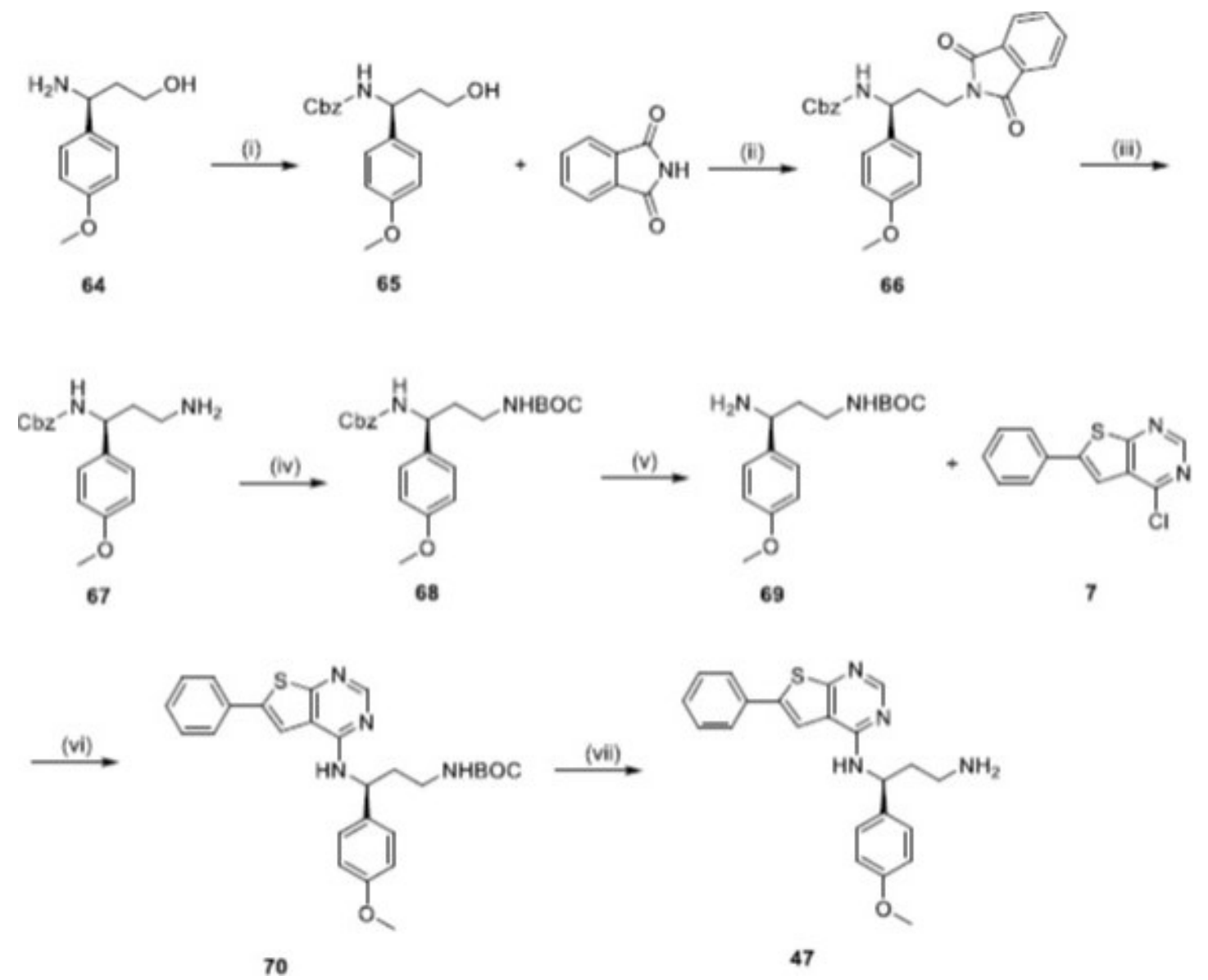

Reagents and conditions: (i) Benzyl chloroformate, $\mathrm{Et}_{3} \mathrm{~N}$, THF, RT, $14 \mathrm{~h}$; (ii) Phthalimide, DIAD, $\mathrm{Ph}_{3} \mathrm{P}$, DCM, RT, 4h; (iii) $\mathrm{NH}_{2} \mathrm{NH}_{2}$, methanol, RT, 6 h; (iv) di-tertbutyl decarbonate, Et 3 N, DCM, RT, 2 h; (v) Pd/C (10 wt\%), $\mathrm{H}_{2}$, methanol, 14 h; (vi) Et3N, reflux, 6 h; (vii) $4 \mathrm{M} \mathrm{HCl}$ in dioxane, DCM, methanol, RT, 4 h. 
Scheme 3-3. Synthesis of amine 48.

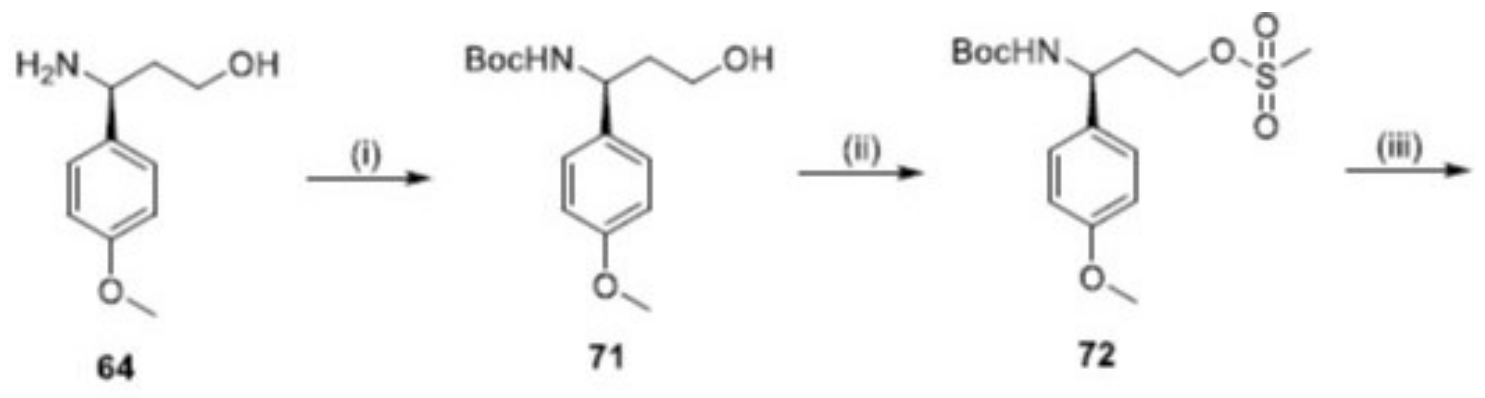<smiles>COc1ccc(C(CCN(C)C)NC(=O)OCc2ccccc2)cc1</smiles>

73
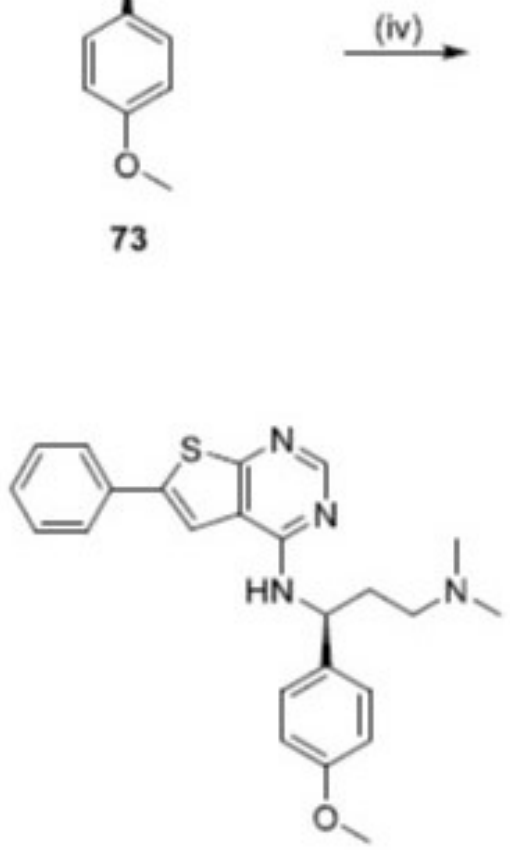

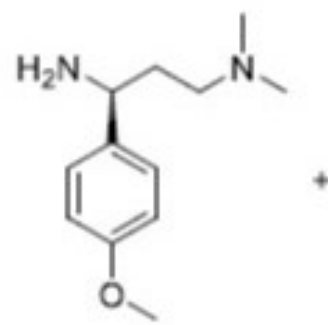

74

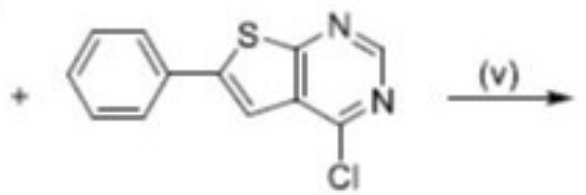

7

48

Reagents and conditions: (i) di-tert-butyl dicarbonate, Et $3 \mathrm{~N}, \mathrm{DCM}, \mathrm{RT}, 2 \mathrm{~h}$; (ii) Methanesulfonyl chloride, $\mathrm{Et}_{3} \mathrm{~N}, \mathrm{DCM}, \mathrm{RT}, 2 \mathrm{~h}$; (iii) Dimethylamine in ethanol, tetrabutylammonium, $150^{\circ} \mathrm{C}$, Microwave, $0.5 \mathrm{~h}$; (iv) $4 \mathrm{M} \mathrm{HCl}$ in dioxane, $\mathrm{DCM}$, methanol, RT, 4 h; (v) Ethanol, $150^{\circ} \mathrm{C}$, Microwave, $1 \mathrm{~h}$. 
Scheme 3-4. Starting from 4-chloro, 6-bromo thienopyrimidine, amination reaction introduced the 4-position side chain and gave the intermediate 76. Suzuki coupling reaction of 76 with the corresponding aryl boronic acids gave the targeted compounds 5053. To remove the 6-phenyl ring and replace it with a methyl motif, 54 was synthesized following the general procedure for the amination of 4-chloro, 6-phenyl thienopyrimidine.

To improve drug clearance of next generation compounds fluorination was explored. Compound $\mathbf{5 5}$ was synthesized with a fluorine atom at the para position of the 6-phenyl moiety, as described in Scheme 3-5. Starting with fluoro-phenylacetaldehyde 82, we carried out ring closure to give the fluorophenylthiophene $\mathbf{8 3}$ in the presence of ethyl-3-cyanopropanoate 4 . The pyrimidine 84 was formed by heating a solution of compound $\mathbf{8 3}$ in excess formamide. Chlorine substituted thienopyrimidine $\mathbf{8 5}$ was prepared in the presence of phosphoryl chloride and was coupled with the amine $\mathbf{5 9}$ to give the targeted analog $\mathbf{5 5}$.

In a scaffold hopping exercise, to replace the sulfur atom and decrease the lipophilicity and increase the stability of $\mathbf{2 5}$, pyrrolopyrimidine and quinazoline scaffolds were identified. ${ }^{134}$ Compounds $\mathbf{5 6}$ and $\mathbf{5 8}$ (ClogP: 3.73 and 4.18, respectively) were synthesized as described in Scheme 3-4. Compound 57 (ClogP: 4.18) was synthesized as described in Scheme 3-6. First, we carried out amination of compound $\mathbf{8 2}$ to give the intermediate 83. Then a Suzuki coupling reaction was prepared with $\mathbf{8 3}$ and the boronic acid $\mathbf{7 7 e}$ to yield targeted compound $\mathbf{5 7 .}$

\section{Anti-H. pylori Activity of New Analogs}

Amine Series. Primary amine compounds 46 and 47 did not show anti-H. pylori activity (H.p. IC $\mathrm{IC}_{50}$ of 2.1 and $2.1 \mu \mathrm{g} / \mathrm{mL}$ respectively, Table 3-1) compared to their corresponding alcohols 25 and 45 (H.p. IC50 of 0.006 and $0.08 \mu \mathrm{g} / \mathrm{mL}$ respectively, Table 3-1). Fluorination to the side chain phenyl ring produced primary amine 31, which showed slightly improved activity and therapeutic index (H.p. $\mathrm{IC}_{50}: 0.54 \mu \mathrm{g} / \mathrm{mL}$ and T. Index: 35.43). The tertiary amine 48 further weakened activity (H.p. IC $50: 12.9 \mu \mathrm{g} / \mathrm{mL}$ ). To reduce lipophilicity, compound $49(\mathrm{Clog} P=3.17$, Table 3-1) was synthesized to replace the para methoxy phenyl motif of 25, with a pyridinyl ring.Compound 49 showed activity but was cytotoxic, resulting in a low therapeutic index $\left(\right.$ H.p. $\mathrm{IC}_{50}: 0.167 \mu \mathrm{g} / \mathrm{mL}$ and T. Index: 92.39, Table 3-1).

Heteroaryl Substitution. Pyridinyl substitution at the 6- position theinopyrimidine was well tolerated. 50, 51 and $\mathbf{5 2}$ show good anti-H. pylori activities ( $\mathrm{IC}_{50}: 0.112 \mu \mathrm{g} / \mathrm{mL}, 0.084 \mu \mathrm{g} / \mathrm{mL}$ and $0.115 \mu \mathrm{g} / \mathrm{mL}$ respectively, Table 3-2). However, these compounds are cytotoxic, and demonstrate low therapeutic indices (T. Index: 55.80, 169.17 and 95.91 respectively, Table 3-2). Compound 53 with a pyrazole ring at the 6position, was less potent and more cytotoxic than the corresponding pyridines (H.p. IC 50 : $0.385 \mu \mathrm{g} / \mathrm{mL}$, Cytotox $\mathrm{IC}_{50}: 6.31 \mu \mathrm{g} / \mathrm{mL}$, Table 3-2). The pyridyl and pyrazole decreased the $\log \mathrm{P}$ of the series by over one Log unit, suggesting a major increase in solubility. 
Scheme 3-4. Synthesis of thienopyrimidine and pyrrolopyrimidine derivatives with modifications at the 6-position.

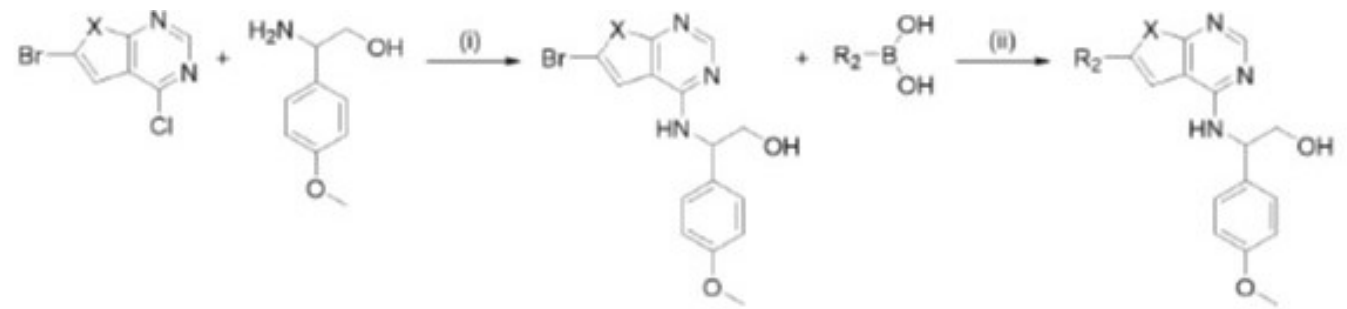

75a: $X=S$

75b: $X=\mathrm{NH}$

75c: $\mathrm{X}=\mathrm{C}=\mathrm{C}$
59 76a: $X=S$

76b: $X=\mathrm{NH}$

76c: $X=C=C$

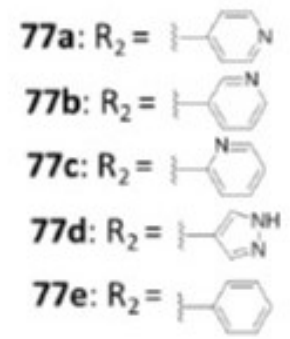

50: $\mathrm{X}=\mathrm{S}, \mathrm{R}_{2}=1-\mathrm{N}$

51: $\mathrm{X}=\mathrm{S}, \mathrm{R}_{2}=$

52: $X=S, R_{2}=$

53: $X=S, R_{2}=1-N$

56: $\mathrm{X}=\mathrm{NH}, \mathrm{R}_{2}=$

58: $X=C=C, R_{2}=$

Reagents and conditions: (i) Ethanol, $150^{\circ} \mathrm{C}$, Microwave, $1 \mathrm{~h}$; (ii) $\mathrm{Pd}(\mathrm{Ph})_{4}, \mathrm{~K}_{2} \mathrm{CO}_{3}$, Dioxane, $70^{\circ} \mathrm{C}, 14 \mathrm{~h}$.

Scheme 3-5. Synthesis of fluorophenyl thienopyrimidine 55.<smiles>CCOC(=O)c1cc(-c2ccc(F)cc2)sc1N</smiles>

78

4

79

80

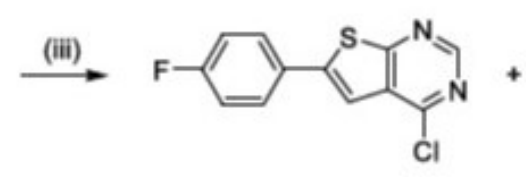<smiles>C1CCCCC1</smiles><smiles>COc1ccc(C(N)CO)cc1</smiles>

59

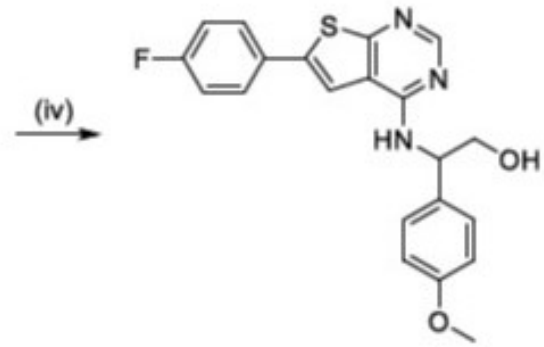

55

Reagents and conditions: (i) Sulfur, Morpholine, Ethanol, $70^{\circ} \mathrm{C}$, Microwave, $20 \mathrm{~min}$, 90\%; (ii) Formamide, reflux, 18 h, 80\%; (iii) POCl3, N,N dimethylaniline, reflux, 14 h, 90\%; (iv) Ethanol, $150^{\circ} \mathrm{C}$, Microwave, 1 h, $93 \%$. 
Scheme 3-6. Synthesis of quinazoline 57.

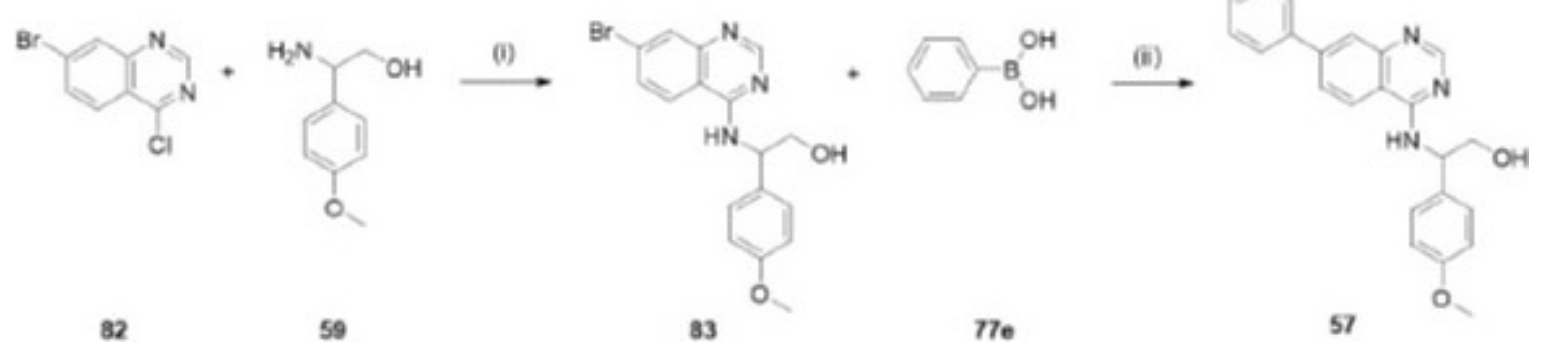

Reagents and conditions: (i) Ethanol, $150^{\circ} \mathrm{C}$, Microwave, $1 \mathrm{~h}$; (ii) $\mathrm{Pd}(\mathrm{Ph})_{4}, \mathrm{~K}_{2} \mathrm{CO}_{3}$, Dioxane, $70^{\circ} \mathrm{C}, 14 \mathrm{~h}$. 
Table 3-1. Thienopyrimidines with modified moieties at the 4-position for improved solubility and reduced lipophilicity.

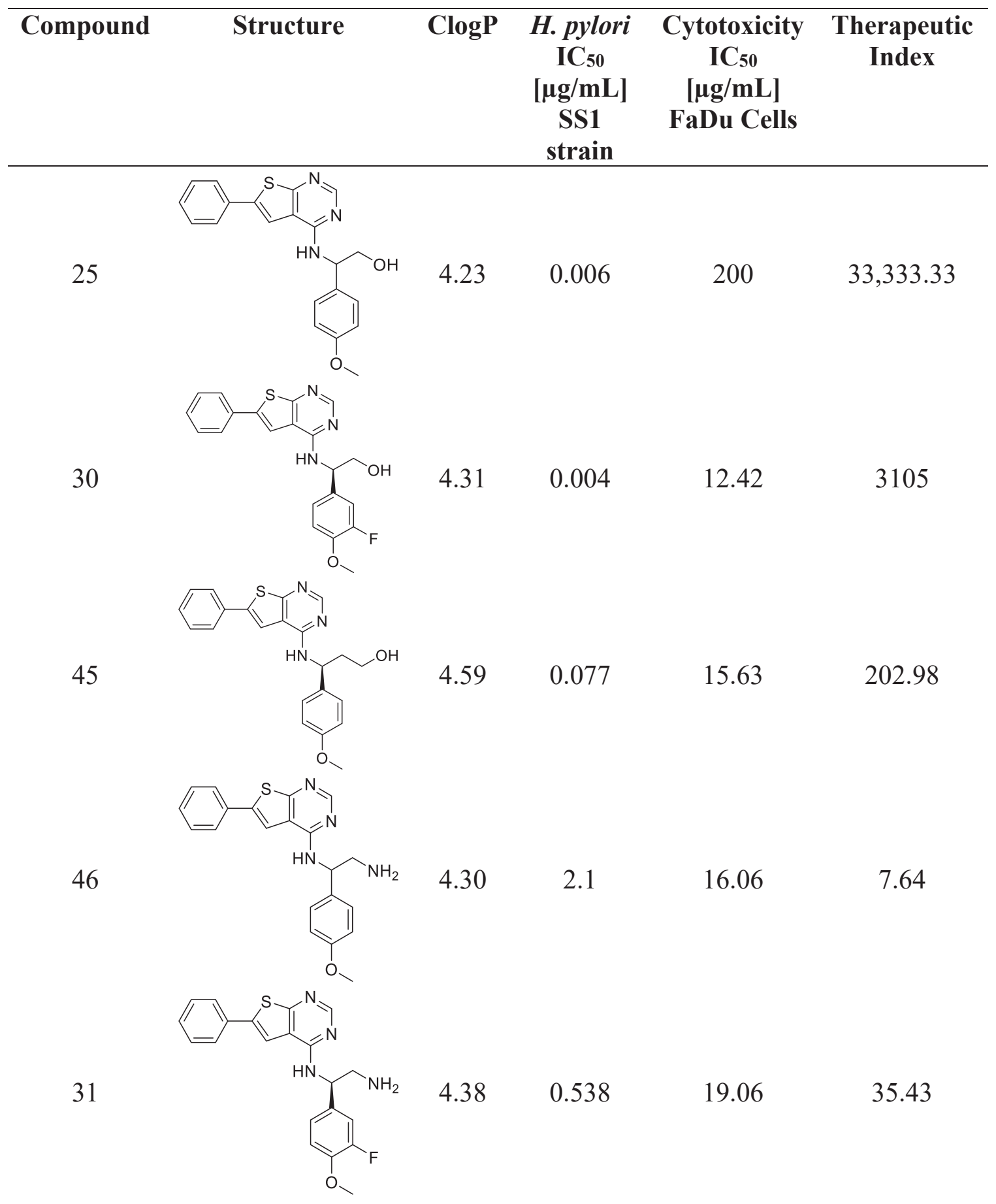


Table 3-1. (Continued).

\begin{tabular}{|c|c|c|c|c|c|}
\hline Compound & Structure & ClogP & $\begin{array}{c}\text { H. pylori } \\
\text { IC50 } \\
\text { [Mg/mL] } \\
\text { SS1 } \\
\text { strain } \\
\end{array}$ & $\begin{array}{c}\text { Cytotoxicity } \\
\text { IC } 50[\mu \mathrm{g} / \mathrm{mL}] \\
\text { FaDu Cells }\end{array}$ & $\begin{array}{c}\text { Therapeutic } \\
\text { Index }\end{array}$ \\
\hline 47 & & 4.67 & 2.09 & 43.75 & 20.93 \\
\hline 48 & & 5.51 & 12.92 & 15.72 & 1.22 \\
\hline 49 & & 3.17 & 0.167 & 15.43 & 92.39 \\
\hline
\end{tabular}


Table 3-2. Thienopyrimidines with modifications at the 4-position.

\begin{tabular}{|c|c|c|c|c|c|}
\hline Compound & Structure & $\mathrm{Clog} P$ & $\begin{array}{c}\text { H. pylori } \\
\text { IC50 } \\
\text { [Mg/mL] } \\
\text { SS1 } \\
\text { strain } \\
\end{array}$ & $\begin{array}{c}\text { Cytotoxicity } \\
\text { IC50 } \\
{[\mu \mathrm{mg} / \mathrm{mL}]} \\
\text { FaDu Cells }\end{array}$ & $\begin{array}{c}\text { Therapeutic } \\
\text { Index }\end{array}$ \\
\hline 50 & & 2.75 & 0.112 & 6.25 & 55.80 \\
\hline 51 & & 2.75 & 0.084 & 14.21 & 169.17 \\
\hline 52 & & 2.96 & 0.115 & 11.03 & 95.91 \\
\hline 53 & & 2.36 & 0.385 & 6.31 & 16.39 \\
\hline 54 & & 3.08 & 0.123 & 34.87 & 283.49 \\
\hline 55 & & 4.37 & 0.016 & 200 & 12,500 \\
\hline
\end{tabular}


To reduce lipophilicity, we synthesized compound 54 (ClogP: 3.08, Table 3-2) with a methyl group replacing the phenyl ring at the 6-position. Compound $\mathbf{5 4}$ shows acceptable activity and a good therapeutic index (H.p. $\mathrm{IC}_{50}: 0.123 \mu \mathrm{g} / \mathrm{mL}$, T. Index: $283.49 \mu \mathrm{g} / \mathrm{mL})$.

Fluorination of Leads. Compound 55, with a fluorine substituted at the 6-phenyl ring, maintained high potency (H.p. $\mathrm{IC}_{50}: 0.016 \mu \mathrm{g} / \mathrm{mL}$, Table 3-2) and an excellent therapeutic window. (T. Index: 12,500, Table 3-2).

Scaffold Hopping Analogs. The pyrrole-pyrimidine compound 56 (H.p. $\mathrm{IC}_{50}$ : $0.065 \mu \mathrm{g} / \mathrm{mL}$, Table 3-3) and the quinazoline compound 57 (H.p. $\mathrm{IC}_{50}: 0.034 \mu \mathrm{g} / \mathrm{mL}$, Table 3-3) demonstrate high potency and acceptable therapeutic indices ( $T$. Index: 454.31 and 730 respectively, Table 3-3). The quinazoline 58 shows reduced potency (H.p. $\mathrm{IC}_{50}: 0.499 \mu \mathrm{g} / \mathrm{mL}$, Table 3-3), suggesting the phenyl ring is preferred at the 7position over the 6-position of the quinazoline core.

\section{ADME Properties of New Leads}

Since, compounds $\mathbf{3 0}, \mathbf{4 5}, \mathbf{5 4}, \mathbf{5 5}, \mathbf{5 6}$ and $\mathbf{5 7}$ demonstrate good therapeutic indices $(>200)$, we proceeded to determine the solubility, the human plasma protein binding percentage and the microsomal stability of these compounds (Table 3-4). Compared to 25, compound 30 shows a slightly improved solubility (Sol: $3 \mu \mathrm{M}$, Table 3-4) and a reduced metabolic stability $\left(\mathrm{t}_{1 / 2}=0.86 \mathrm{hr}\right.$, Table 3-4). To our surprise, compound 45 show good solubility (Sol: $63.7 \mu \mathrm{g} / \mathrm{mL}$, Table 3-4), a good HPPB\% (96.71, Table 3-4) and an acceptable metabolic stability $\left(\mathrm{t}_{1 / 2}=0.99 \mathrm{hr}\right.$, Table 3-4). When comparing compound 25 and 55 (Table 3-4), the introduction of a fluorine atom at the para position of the 6-phenyl ring, significantly improve the microsomal stability $\left(\mathrm{t}_{1 / 2}: 13.34 \mathrm{hr}\right.$, Table 3-4) but decreased the solubility. The replacement of the phenyl substituent at the 6 position with a methyl motif, as exemplified by compound $\mathbf{5 4}$, significantly improves the solubility (Sol: $69.8 \mu \mathrm{M}$, Table 3-4) and reduces the plasma protein binding (HPPB\%: 95.9, Table 3-4). However, this replacement of phenyl with a methyl leads to a major reduction of metabolic stability ( $\mathrm{t}_{1 / 2}: 0.03 \mathrm{hr}$, Table 3-4). The pyrrolopyrimidine analog 56 demonstrates an improved solubility (Sol: $20.1 \mu \mathrm{M}$, Table 3-4) and a reduced plasma protein binding (HPPB\%: 97.9, Table 3-4). Although 57 is less bound to plasma protein (HPPB\%: 98.1, Table 3-4), it does not demonstrate a significant improvement in solubility (Sol: $2.4 \mu \mathrm{M}$, Table 3-4).

\section{Conclusion}

In conclusion, we have introduced targeted chemical modifications to improve the in vitro pharmacokinetic properties of the lead compound 25. Although it demonstrates a high potency $(\mathrm{H} . \mathrm{p}$. IC50: $0.006 \mathrm{ug} / \mathrm{mL}), 25$ lacks in vivo efficacy due to poor solubility, high protein binding and high lipophilicity. These factors limit the amount of free drug concentration that reaches the H. pylori site of infection. Efforts to improve these in vitro 
Table 3-3. Pyrrolo[2,3-d]pyrimidine and quinazolines synthesized for improved solubility.

\begin{tabular}{|c|c|c|c|c|c|}
\hline Compound & Structure & $\mathrm{Clog} P$ & $\begin{array}{c}\text { H. pylori } \\
\text { IC50 } \\
{[\mu \mathrm{g} / \mathrm{mL}]} \\
\text { SS1 } \\
\text { strain } \\
\end{array}$ & $\begin{array}{c}\text { Cytotoxicity } \\
\text { IC50 } \\
{[\mu \mathrm{g} / \mathrm{mL}]} \\
\text { FaDu Cells }\end{array}$ & $\begin{array}{l}\text { Therapeutic } \\
\text { Index }\end{array}$ \\
\hline 56 & & 3.73 & 0.065 & 29.53 & 454.31 \\
\hline 57 & & 4.18 & 0.034 & 24.82 & 730 \\
\hline 58 & & 4.18 & 0.499 & 32 & 64.13 \\
\hline
\end{tabular}


Table 3-4. ADME properties of selected compounds.

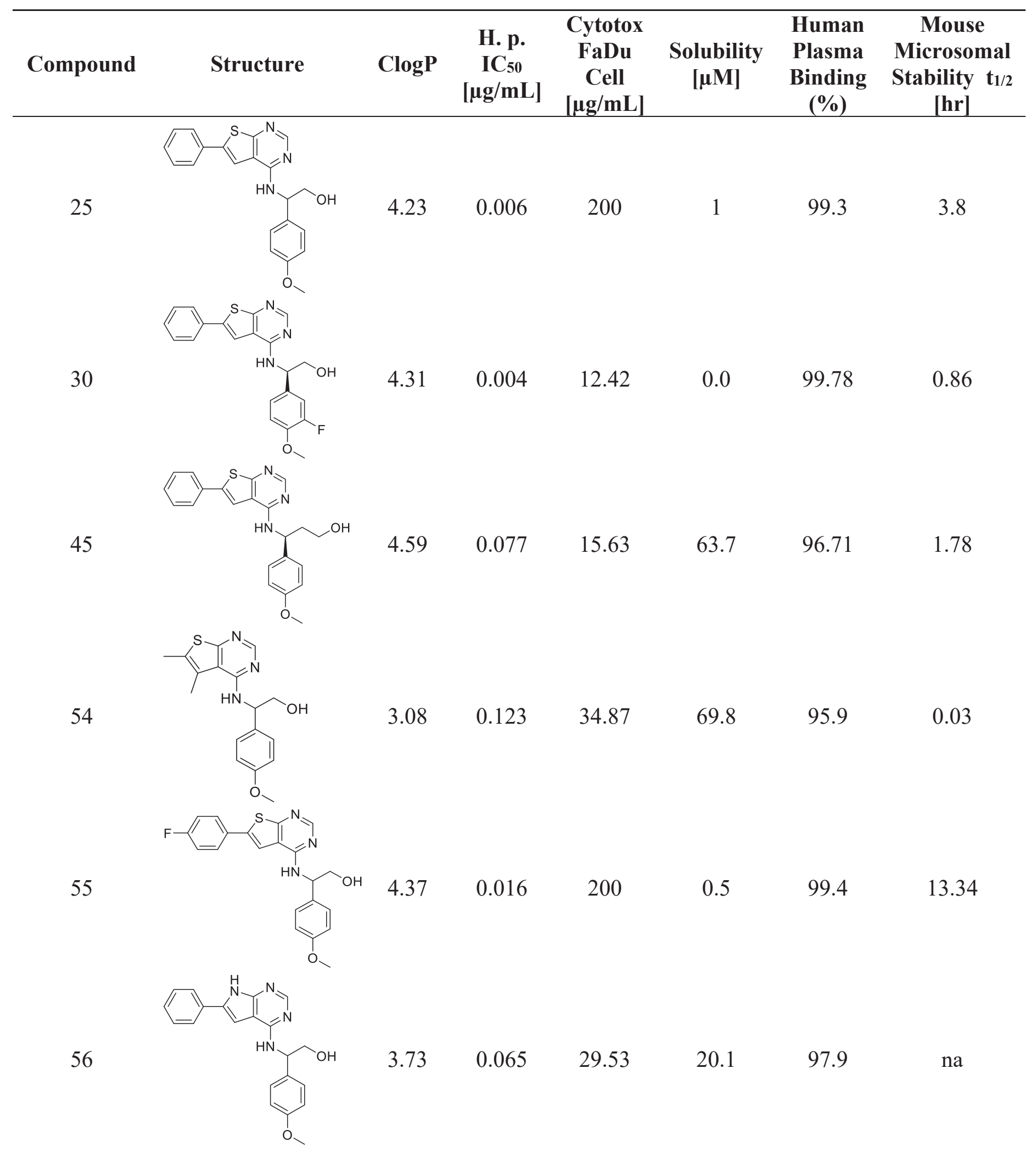


Table 3-4. (Continued).

\begin{tabular}{|c|c|c|c|c|c|c|c|}
\hline Compound & Structure & $C \log P$ & $\begin{array}{c}\text { H. p. } \\
\text { IC } 50 \\
{[\mu g / m L]}\end{array}$ & $\begin{array}{c}\text { Cytotox } \\
\text { FaDu } \\
\text { Cell } \\
{[\mu \mathrm{g} / \mathrm{mL}]}\end{array}$ & $\begin{array}{l}\text { Solubility } \\
\qquad[\mu \mathrm{M}]\end{array}$ & $\begin{array}{c}\text { Human } \\
\text { Plasma } \\
\text { Binding } \\
(\%) \\
\end{array}$ & $\begin{array}{c}\text { Mouse } \\
\text { Microsomal } \\
\text { Stability t } \text { t }_{1 / 2} \\
{[\mathrm{hr}]} \\
\end{array}$ \\
\hline 57 & & 4.18 & 0.034 & 24.82 & 2.4 & 98.1 & na \\
\hline
\end{tabular}


pharmacokinetic properties revealed compound 56, which demonstrates an improved solubility and is less bound to plasma protein. The removal of the sulfur greatly improves the solubility and the plasma protein binding. Para-fluoro substitution at the 6-phenyl group significantly improves the microsomal stability of the series. The pyrrolopyrimidine scaffold is a promising core in the optimization of the ADME properties, to discover a candidate for in vivo efficacy studies in the H. pylori murine model. 


\section{CHAPTER 4. CONCLUSION}

H. pylori is the causative agent of gastritis, peptic ulcers and gastric cancer. The recommended therapy for $H$. pylori infections is the triple therapy which consists of a PPI, and two antibiotics including clarithromycin and amoxicillin. The rate of successful treatment with the triple therapy has dropped below $80 \%$, due to the global rise of resistance against these antibiotics. The World Health Organization lists $H$. pylori as a high priority drug-resistant bacterium. A narrow-spectrum drug is needed to address the drug resistance challenge and preserve these broad-spectrum antibiotics for the treatment of other types of infections. Advances in whole genome sequencing provide an opportunity to discovery new chemical matter. Recent transposon mutagenesis studies have revealed $H$. pylori specific genes that enable the pathogen to grow in the stomach. The existence of these genes suggests that there are novel and unique targets that can explored for the development of novel therapies against $H$. pylori infections. Phenotypic screening for selective compounds against $H$. pylori is a promising approach to unveil these targets.

In an effort to discover a new chemical matter, we conducted a high-throughput screen of 219,197 compounds. Positive hits were counter-screened against a number of gut commensals, to eliminate promiscuous compounds. Two thienopyrimidines emerged from this screen and were explored through the synthesis of 29 analogs and the structure activity relationship (SAR) studies that merged elements from both hits. The SAR revealed several potent compounds, in low $\mu \mathrm{g} / \mathrm{mL}$-range of activity, including compound 25 (H.p. $\mathrm{IC}_{50}: 0.006 \mu \mathrm{g} / \mathrm{mL}$ ). Mode of action studies that involved the generation of mutants, revealed NuoD, a subunit of $H$. pylori's respiratory Complex 1, as the putative target of the thienopyrimidine. Complex I is an enzyme that catalyzes the first step of oxidative phosphorylation.

Targeting oxidative phosphorylation has been proven to be beneficial in the clinic, as exemplified by metformin, a drug that is used for the treatment of diabetes. The inhibition of oxidative phosphorylation is becoming an important approach in the development of therapies for the treatment of AML and solid tumors. Additionally, NuoD has been found to be essential for $H$. pylori but not for most of other bacteria including $E$. coli. These realizations suggest that the thienopyrimidines are a promising series of compounds, worth further optimization to fulfill the eradication of $H$. pylori in the in vivo murine model. The lead thienopyrimidine compound 25 eradicates $H$. pylori in the ex vivo model but lacks in vivo efficacy, suggesting the optimization of pharmacokinetic properties of the lead compound 25 is required.

To address the limitations of $\mathbf{2 5}$, we have synthesized 16 additional compounds. At $50 \mathrm{mg} / \mathrm{kg}$, compound 25 shows good exposure (AUC: $31907.71 \mathrm{hr} * \mathrm{ng} / \mathrm{mL}$ ) with a high volume of distribution (Vss: $6.28 \mathrm{~L} / \mathrm{Kg}$ ), suggesting that 25 (Clogp: 4.23 ) is too lipophilic and is being trapped in fatty tissues. 25 also shows poor solubility (Sol: 0.1 $\mu \mathrm{M}$ ) and high plasma protein binding (HPPB: $99.3 \%$ ), suggesting not enough of free drug concentration is able to reach the site of infection, in the mucus layer of the 
stomach. New compounds include amine derivatives of $\mathbf{2 5}$ and other lead compounds such as 30 and 45, to improve the solubility of the series. Scaffold hopping exercise that aimed to remove the lipophilic sulfur atom, revealed pyrrolopyrimidine and quinazoline scaffolds. The pyrrolopyrimidine analog 56 is less lipophilic (ClogP: 3.73), demonstrates an improved solubility (Sol: $20.1 \mu \mathrm{M}$ ) and is less bound to plasma protein (HPPB: 97.9 \%) compared to lead compound $\mathbf{2 5}$. Compound 56 is a promising compound, in the lead optimization efforts toward the in vivo proof of concept studies in the $H$. pylori murine model. 6-pyridinyl substituted derivative of pyrrolopyrimidine $\mathbf{5 6}$ will likely lead to improved solubility and decreased plasma protein binding. Fluorination of the 6-aryl ring will prolong the half-life of drug in vivo. Substitution of the (R)-3-amino-3-(4methoxyphenyl) propanol motif, identified with compound 45, at the 4-position of the pyrrolopyrimidine core could lead to a series with improved solubility and low plasma protein binding. 


\section{LIST OF REFERENCES}

1. Marcus, E. A.; Sachs, G.; Scott, D. R., Eradication of Helicobacter pylori Infection. Curr Gastroenterol Rep 2016, 18 (7), 33.

2. Hooi, J. K. Y.; Lai, W. Y.; Ng, W. K.; Suen, M. M. Y.; Underwood, F. E.; Tanyingoh, D.; Malfertheiner, P.; Graham, D. Y.; Wong, V. W. S.; Wu, J. C. Y.; Chan, F. K. L.; Sung, J. J. Y.; Kaplan, G. G.; Ng, S. C., Global Prevalence of Helicobacter pylori Infection: Systematic Review and Meta-Analysis. Gastroenterology 2017, 153 (2), 420-29.

3. Centers for Disease Control and Prevention. www.cdc.gov; accessed June 12, 2017

4. Pakodi, F.; Abdel-Salam, O. M.; Debreceni, A.; Mozsik, G., Helicobacter pylori. One bacterium and a broad spectrum of human disease! An overview. $J$ Physiol Paris 2000, 94 (2), 139-52.

5. Keilberg, D.; Ottemann, K. M., How Helicobacter pylori senses, targets and interacts with the gastric epithelium. Environ Microbiol 2016, 18 (3), 791-806.

6. Wotherspoon, A. C.; Doglioni, C.; de Boni, M.; Spencer, J.; Isaacson, P. G., Antibiotic treatment for low-grade gastric MALT lymphoma. Lancet 1994, 343 (8911), 1503.

7. World Health Organization. www.who.int; accessed June 13, 2017

8. Ferlay, J.; Shin, H. R.; Bray, F.; Forman, D.; Mathers, C.; Parkin, D. M., Estimates of worldwide burden of cancer in 2008: GLOBOCAN 2008. Int $J$ Cancer 2010, 127 (12), 2893-917.

9. The nobel prize. www.nobelprize.org/prizes/medecine/2005/pressrelease; accessed June 14, 2017

10. Chey, W. D.; Wong, B. C.; Practice Parameters Committee of the American College of, G., American College of Gastroenterology guideline on the management of Helicobacter pylori infection. Am J Gastroenterol 2007, 102 (8), 1808-25.

11. Chey, W. D.; Leontiadis, G. I.; Howden, C. W.; Moss, S. F., ACG Clinical Guideline: Treatment of Helicobacter pylori Infection. Am J Gastroenterol 2017, $112(2), 212-239$.

12. Ford, A. C.; Delaney, B. C.; Forman, D.; Moayyedi, P., Eradication therapy for peptic ulcer disease in Helicobacter pylori positive patients. Cochrane Database Syst Rev 2006, (2), CD003840.

13. Gisbert, J. P.; Khorrami, S.; Carballo, F.; Calvet, X.; Gene, E.; Dominguez-Munoz, J. E., H. pylori eradication therapy vs. antisecretory non-eradication therapy (with or without long-term maintenance antisecretory therapy) for the prevention of recurrent bleeding from peptic ulcer. Cochrane Database Syst Rev 2004, (2), CD004062.

14. Leodolter, A.; Kulig, M.; Brasch, H.; Meyer-Sabellek, W.; Willich, S. N.; Malfertheiner, P., A meta-analysis comparing eradication, healing and relapse rates in patients with Helicobacter pylori-associated gastric or duodenal ulcer. Aliment Pharmacol Ther 2001, 15 (12), 1949-58.

15. Wong, B. C.; Lam, S. K.; Wong, W. M.; Chen, J. S.; Zheng, T. T.; Feng, R. E.; Lai, K. C.; Hu, W. H.; Yuen, S. T.; Leung, S. Y.; Fong, D. Y.; Ho, J.; Ching, C. K.; 
Chen, J. S.; China Gastric Cancer Study, G., Helicobacter pylori eradication to prevent gastric cancer in a high-risk region of China: a randomized controlled trial. JAMA 2004, 291 (2), 187-94.

16. Wotherspoon, A. C.; Doglioni, C.; Diss, T. C.; Pan, L.; Moschini, A.; de Boni, M.; Isaacson, P. G., Regression of primary low-grade B-cell gastric lymphoma of mucosa-associated lymphoid tissue type after eradication of Helicobacter pylori. Lancet 1993, 342 (8871), 575-7.

17. Stathis, A.; Chini, C.; Bertoni, F.; Proserpio, I.; Capella, C.; Mazzucchelli, L.; Pedrinis, E.; Cavalli, F.; Pinotti, G.; Zucca, E., Long-term outcome following Helicobacter pylori eradication in a retrospective study of 105 patients with localized gastric marginal zone B-cell lymphoma of MALT type. Ann Oncol 2009, 20 (6), 1086-93.

18. Nakamura, S.; Sugiyama, T.; Matsumoto, T.; Iijima, K.; Ono, S.; Tajika, M.; Tari, A.; Kitadai, Y.; Matsumoto, H.; Nagaya, T.; Kamoshida, T.; Watanabe, N.; Chiba, T.; Origasa, H.; Asaka, M.; Group, J. G. S., Long-term clinical outcome of gastric MALT lymphoma after eradication of Helicobacter pylori: a multicentre cohort follow-up study of 420 patients in Japan. Gut 2012, 61 (4), 507-13.

19. Malfertheiner, P.; Megraud, F.; O'Morain, C. A.; Atherton, J.; Axon, A. T.; Bazzoli, F.; Gensini, G. F.; Gisbert, J. P.; Graham, D. Y.; Rokkas, T.; El-Omar, E. M.; Kuipers, E. J.; European Helicobacter Study, G., Management of Helicobacter pylori infection--the Maastricht IV/ Florence Consensus Report. Gut 2012, 61 (5), 646-64.

20. Marcus, E. A.; Inatomi, N.; Nagami, G. T.; Sachs, G.; Scott, D. R., The effects of varying acidity on Helicobacter pylori growth and the bactericidal efficacy of ampicillin. Aliment Pharmacol Ther 2012, 36 (10), 972-9.

21. McNulty, C. A.; Lasseter, G.; Shaw, I.; Nichols, T.; D'Arcy, S.; Lawson, A. J.; Glocker, E., Is Helicobacter pylori antibiotic resistance surveillance needed and how can it be delivered? Aliment Pharmacol Ther 2012, 35 (10), 1221-30.

22. Vianna, J. S.; Ramis, I. B.; Ramos, D. F.; A, V. O. N. G.; Silva, P. E., Drug Resistance in Helicobacter Pylori. Arq Gastroenterol 2016, 53 (4), 215-223.

23. Gisbert, J. P.; Gonzalez, L.; Calvet, X.; Garcia, N.; Lopez, T.; Roque, M.; Gabriel, R.; Pajares, J. M., Proton pump inhibitor, clarithromycin and either amoxycillin or nitroimidazole: a meta-analysis of eradication of Helicobacter pylori. Aliment Pharmacol Ther 2000, 14 (10), 1319-28.

24. Sun, Q.; Liang, X.; Zheng, Q.; Liu, W.; Xiao, S.; Gu, W.; Lu, H., High efficacy of 14-day triple therapy-based, bismuth-containing quadruple therapy for initial Helicobacter pylori eradication. Helicobacter 2010, 15 (3), 233-8.

25. Marcus, E. A.; Sachs, G.; Scott, D. R., Colloidal bismuth subcitrate impedes proton entry into Helicobacter pylori and increases the efficacy of growth-dependent antibiotics. Aliment Pharmacol Ther 2015, 42 (7), 922-33.

26. Li, B. Z.; Threapleton, D. E.; Wang, J. Y.; Xu, J. M.; Yuan, J. Q.; Zhang, C.; Li, P.; Ye, Q. L.; Guo, B.; Mao, C.; Ye, D. Q., Comparative effectiveness and tolerance of treatments for Helicobacter pylori: systematic review and network meta-analysis. BMJ 2015, 351, h4052.

27. Luther, J.; Higgins, P. D.; Schoenfeld, P. S.; Moayyedi, P.; Vakil, N.; Chey, W. D., Empiric quadruple vs. triple therapy for primary treatment of Helicobacter pylori 
infection: Systematic review and meta-analysis of efficacy and tolerability. Am J Gastroenterol 2010, 105 (1), 65-73.

28. Lind, T.; Veldhuyzen van Zanten, S.; Unge, P.; Spiller, R.; Bayerdorffer, E.; O'Morain, C.; Bardhan, K. D.; Bradette, M.; Chiba, N.; Wrangstadh, M.; Cederberg, C.; Idstrom, J. P., Eradication of Helicobacter pylori using one-week triple therapies combining omeprazole with two antimicrobials: the MACH I Study. Helicobacter 1996, 1 (3), 138-44.

29. Duck, W. M.; Sobel, J.; Pruckler, J. M.; Song, Q.; Swerdlow, D.; Friedman, C.; Sulka, A.; Swaminathan, B.; Taylor, T.; Hoekstra, M.; Griffin, P.; Smoot, D.; Peek, R.; Metz, D. C.; Bloom, P. B.; Goldschmidt, S.; Parsonnet, J.; Triadafilopoulos, G.; Perez-Perez, G. I.; Vakil, N.; Ernst, P.; Czinn, S.; Dunne, D.; Gold, B. D., Antimicrobial resistance incidence and risk factors among Helicobacter pyloriinfected persons, United States. Emerg Infect Dis 2004, 10 (6), 1088-94.

30. Tveit, A. H.; Bruce, M. G.; Bruden, D. L.; Morris, J.; Reasonover, A.; Hurlburt, D. A.; Hennessy, T. W.; McMahon, B., Alaska sentinel surveillance study of Helicobacter pylori isolates from Alaska Native persons from 2000 to 2008. J Clin Microbiol 2011, 49 (10), 3638-43.

31. Shiota, S.; Reddy, R.; Alsarraj, A.; El-Serag, H. B.; Graham, D. Y., Antibiotic Resistance of Helicobacter pylori Among Male United States Veterans. Clin Gastroenterol Hepatol 2015, 13 (9), 1616-24.

32. Hori, K.; Miwa, H.; Matsumoto, T., Efficacy of 2-week, second-line Helicobacter pylori eradication therapy using rabeprazole, amoxicillin, and metronidazole for the Japanese population. Helicobacter 2011, 16 (3), 234-40.

33. Eng, N. F.; Ybazeta, G.; Chapman, K.; Fraleigh, N. L.; Letto, R.; Altman, E.; DiazMitoma, F., Antimicrobial susceptibility of Canadian isolates of Helicobacter pylori in Northeastern Ontario. Can J Infect Dis Med Microbiol 2015, 26 (3), 137-44.

34. Smith, S. M.; O'Morain, C.; McNamara, D., Antimicrobial susceptibility testing for Helicobacter pylori in times of increasing antibiotic resistance. World $J$ Gastroenterol 2014, 20 (29), 9912-21.

35. Lambert, J. R.; Midolo, P., The actions of bismuth in the treatment of Helicobacter pylori infection. Aliment Pharmacol Ther 1997, 11 Suppl 1, 27-33.

36. Taneike, I.; Goshi, S.; Tamura, Y.; Wakisaka-Saito, N.; Matsumori, N.; Yanase, A.; Shimizu, T.; Yamashiro, Y.; Toyoda, S.; Yamamoto, T., Emergence of clarithromycin-resistant Helicobacter pylori (CRHP) with a high prevalence in children compared with their parents. Helicobacter 2002, 7 (5), 297-305

37. Gisbert, J. P.; Romano, M.; Gravina, A. G.; Solis-Munoz, P.; Bermejo, F.; MolinaInfante, J.; Castro-Fernandez, M.; Ortuno, J.; Lucendo, A. J.; Herranz, M.; Modolell, I.; Del Castillo, F.; Gomez, J.; Barrio, J.; Velayos, B.; Gomez, B.; Dominguez, J. L.; Miranda, A.; Martorano, M.; Algaba, A.; Pabon, M.; Angueira, T.; Fernandez-Salazar, L.; Federico, A.; Marin, A. C.; McNicholl, A. G., Helicobacter pylori second-line rescue therapy with levofloxacin- and bismuthcontaining quadruple therapy, after failure of standard triple or non-bismuth quadruple treatments. Aliment Pharmacol Ther 2015, 41 (8), 768-75.

38. Wong, B. C.; Lam, S. K.; Wong, W. M.; Chen, J. S.; Zheng, T. T.; Feng, R. E.; Lai, K. C.; Hu, W. H.; Yuen, S. T.; Leung, S. Y.; Fong, D. Y.; Ho, J.; Ching, C. K.; Chen, J. S.; China Gastric Cancer Study, G., Helicobacter pylori eradication to 
prevent gastric cancer in a high-risk region of China: a randomized controlled trial. JAMA 2004, 291 (2), 187-94.

39. Goodwin, A.; Kersulyte, D.; Sisson, G.; Veldhuyzen van Zanten, S. J.; Berg, D. E.; Hoffman, P. S., Metronidazole resistance in Helicobacter pylori is due to null mutations in a gene (rdxA) that encodes an oxygen-insensitive NADPH nitroreductase. Mol Microbiol 1998, 28 (2), 383-93.

40. Tsugawa, H.; Suzuki, H.; Muraoka, H.; Ikeda, F.; Hirata, K.; Matsuzaki, J.; Saito, Y.; Hibi, T., Enhanced bacterial efflux system is the first step to the development of metronidazole resistance in Helicobacter pylori. Biochem Biophys Res Commun 2011, 404 (2), 656-60.

41. Kutschke, A.; de Jonge, B. L., Compound efflux in Helicobacter pylori. Antimicrob Agents Chemother 2005, 49 (7), 3009-10.

42. Muri, E. M.; Williamson, J. S., Anti-Helicobacter pylori agents. An update. Mini Rev Med Chem 2004, 4 (2), 201-6.

43. Brown, L. M., Helicobacter pylori: epidemiology and routes of transmission. Epidemiol Rev 2000, 22 (2), 283-97.

44. Parsonnet, J.; Shmuely, H.; Haggerty, T., Fecal and oral shedding of Helicobacter pylori from healthy infected adults. JAMA 1999, 282 (23), 2240-5.

45. Goodman, K. J.; Correa, P.; Tengana Aux, H. J.; Ramirez, H.; DeLany, J. P.; Guerrero Pepinosa, O.; Lopez Quinones, M.; Collazos Parra, T., Helicobacter pylori infection in the Colombian Andes: a population-based study of transmission pathways. Am J Epidemiol 1996, 144 (3), 290-9.

46. Schreiber, S.; Konradt, M.; Groll, C.; Scheid, P.; Hanauer, G.; Werling, H. O.; Josenhans, C.; Suerbaum, S., The spatial orientation of Helicobacter pylori in the gastric mucus. Proc Natl Acad Sci U S A 2004, 101 (14), 5024-9.

47. Rolig, A. S.; Shanks, J.; Carter, J. E.; Ottemann, K. M., Helicobacter pylori requires TlpD-driven chemotaxis to proliferate in the antrum. Infect Immun 2012, 80 (10), 3713-20.

48. Howitt, M. R.; Lee, J. Y.; Lertsethtakarn, P.; Vogelmann, R.; Joubert, L. M.; Ottemann, K. M.; Amieva, M. R., ChePep controls Helicobacter pylori Infection of the gastric glands and chemotaxis in the Epsilonproteobacteria. MBio 2011, 2 (4).

49. Meyer-Rosberg, K.; Scott, D. R.; Rex, D.; Melchers, K.; Sachs, G., The effect of environmental $\mathrm{pH}$ on the proton motive force of Helicobacter pylori. Gastroenterology 1996, 111 (4), 886-900.

50. Marcus, E. A.; Moshfegh, A. P.; Sachs, G.; Scott, D. R., The periplasmic alphacarbonic anhydrase activity of Helicobacter pylori is essential for acid acclimation. $J$ Bacteriol 2005, 187 (2), 729-38.

51. Scott, D. R.; Marcus, E. A.; Weeks, D. L.; Lee, A.; Melchers, K.; Sachs, G., Expression of the Helicobacter pylori ureI gene is required for acidic $\mathrm{pH}$ activation of cytoplasmic urease. Infect Immun 2000, 68 (2), 470-7.

52. Weeks, D. L.; Eskandari, S.; Scott, D. R.; Sachs, G., A H+-gated urea channel: the link between Helicobacter pylori urease and gastric colonization. Science 2000, 287 (5452), 482-5.

53. Larhed, A. W.; Artursson, P.; Grasjo, J.; Bjork, E., Diffusion of drugs in native and purified gastrointestinal mucus. J Pharm Sci-Us 1997, 86 (6), 660-665. 
54. Croxen, M. A.; Sisson, G.; Melano, R.; Hoffman, P. S., The Helicobacter pylori chemotaxis receptor TlpB (HP0103) is required for $\mathrm{pH}$ taxis and for colonization of the gastric mucosa. J Bacteriol 2006, 188 (7), 2656-65.

55. Martinez, L. E.; Hardcastle, J. M.; Wang, J.; Pincus, Z.; Tsang, J.; Hoover, T. R.; Bansil, R.; Salama, N. R., Helicobacter pylori strains vary cell shape and flagellum number to maintain robust motility in viscous environments. Mol Microbiol 2016, 99 (1), 88-110.

56. Celli, J. P.; Turner, B. S.; Afdhal, N. H.; Keates, S.; Ghiran, I.; Kelly, C. P.; Ewoldt, R. H.; McKinley, G. H.; So, P.; Erramilli, S.; Bansil, R., Helicobacter pylori moves through mucus by reducing mucin viscoelasticity. Proc Natl Acad Sci U S A 2009, 106 (34), 14321-6.

57. Mizote, T.; Yoshiyama, H.; Nakazawa, T., Urease-independent chemotactic responses of Helicobacter pylori to urea, urease inhibitors, and sodium bicarbonate. Infect Immun 1997, 65 (4), 1519-21.

58. Sanders, L.; Andermann, T. M.; Ottemann, K. M., A supplemented soft agar chemotaxis assay demonstrates the Helicobacter pylori chemotactic response to zinc and nickel. Microbiology 2013, 159 (Pt 1), 46-57.

59. Huang, J. Y.; Sweeney, E. G.; Sigal, M.; Zhang, H. C.; Remington, S. J.; Cantrell, M. A.; Kuo, C. J.; Guillemin, K.; Amieva, M. R., Chemodetection and Destruction of Host Urea Allows Helicobacter pylori to Locate the Epithelium. Cell Host Microbe 2015, 18 (2), 147-56.

60. Aihara, E.; Closson, C.; Matthis, A. L.; Schumacher, M. A.; Engevik, A. C.; Zavros, Y.; Ottemann, K. M.; Montrose, M. H., Motility and chemotaxis mediate the preferential colonization of gastric injury sites by Helicobacter pylori. PLoS Pathog 2014, 10 (7), e1004275.

61. Jimenez-Pearson, M. A.; Delany, I.; Scarlato, V.; Beier, D., Phosphate flow in the chemotactic response system of Helicobacter pylori. Microbiology 2005, $151(\mathrm{Pt}$ 10), 3299-311.

62. Cerda, O. A.; Nunez-Villena, F.; Soto, S. E.; Ugalde, J. M.; Lopez-Solis, R.; Toledo, H., tlpA gene expression is required for arginine and bicarbonate chemotaxis in Helicobacter pylori. Biol Res 2011, 44 (3), 277-82.

63. Rader, B. A.; Wreden, C.; Hicks, K. G.; Sweeney, E. G.; Ottemann, K. M.; Guillemin, K., Helicobacter pylori perceives the quorum-sensing molecule AI-2 as a chemorepellent via the chemoreceptor TlpB. Microbiology 2011, 157 (Pt 9), 2445-55.

64. Boncristiano, M.; Paccani, S. R.; Barone, S.; Ulivieri, C.; Patrussi, L.; Ilver, D.; Amedei, A.; D'Elios, M. M.; Telford, J. L.; Baldari, C. T., The Helicobacter pylori vacuolating toxin inhibits $\mathrm{T}$ cell activation by two independent mechanisms. J Exp Med 2003, 198 (12), 1887-97.

65. Backert, S.; Clyne, M.; Tegtmeyer, N., Molecular mechanisms of gastric epithelial cell adhesion and injection of CagA by Helicobacter pylori. Cell Commun Signal 2011, 9, 28.

66. Kim, I. J.; Blanke, S. R., Remodeling the host environment: modulation of the gastric epithelium by the Helicobacter pylori vacuolating toxin (VacA). Front Cell Infect Microbiol 2012, 2, 37. 
67. Backert, S.; Tegtmeyer, N., Type IV Secretion and Signal Transduction of Helicobacter pylori CagA through Interactions with Host Cell Receptors. Toxins (Basel) 2017, 9 (4).

68. Keates, S.; Hitti, Y. S.; Upton, M.; Kelly, C. P., Helicobacter pylori infection activates NF-kappa B in gastric epithelial cells. Gastroenterology 1997, 113 (4), 1099-109.

69. Suzuki, M.; Mimuro, H.; Kiga, K.; Fukumatsu, M.; Ishijima, N.; Morikawa, H.; Nagai, S.; Koyasu, S.; Gilman, R. H.; Kersulyte, D.; Berg, D. E.; Sasakawa, C., Helicobacter pylori CagA phosphorylation-independent function in epithelial proliferation and inflammation. Cell Host Microbe 2009, 5 (1), 23-34.

70. Murata-Kamiya, N.; Kurashima, Y.; Teishikata, Y.; Yamahashi, Y.; Saito, Y.; Higashi, H.; Aburatani, H.; Akiyama, T.; Peek, R. M., Jr.; Azuma, T.; Hatakeyama, M., Helicobacter pylori CagA interacts with E-cadherin and deregulates the betacatenin signal that promotes intestinal transdifferentiation in gastric epithelial cells. Oncogene 2007, 26 (32), 4617-26.

71. Polk, D. B.; Peek, R. M., Jr., Helicobacter pylori: gastric cancer and beyond. Nat Rev Cancer 2010, 10 (6), 403-14.

72. Szabo, I.; Brutsche, S.; Tombola, F.; Moschioni, M.; Satin, B.; Telford, J. L.; Rappuoli, R.; Montecucco, C.; Papini, E.; Zoratti, M., Formation of anion-selective channels in the cell plasma membrane by the toxin VacA of Helicobacter pylori is required for its biological activity. EMBO J 1999, 18 (20), 5517-27.

73. Cover, T. L.; Krishna, U. S.; Israel, D. A.; Peek, R. M., Jr., Induction of gastric epithelial cell apoptosis by Helicobacter pylori vacuolating cytotoxin. Cancer Res 2003, 63 (5), 951-7.

74. Rassow, J.; Meinecke, M., Helicobacter pylori VacA: a new perspective on an invasive chloride channel. Microbes Infect 2012, 14 (12), 1026-33.

75. Gebert, B.; Fischer, W.; Weiss, E.; Hoffmann, R.; Haas, R., Helicobacter pylori vacuolating cytotoxin inhibits T lymphocyte activation. Science 2003, 301 (5636), 1099-102.

76. Morales-Guerrero, S.; Mucito-Varela, E.; Aguilar-Gutierrez, G. R.; Lopez-vidal, Y.; Castillo-Rojas, G., The role of CagA protein signaling in gastric carcinogenesisCagA signaling in gastric carcinogenesis. Current Topics in Gastritis, 2012

77. Tomb, J. F.; White, O.; Kerlavage, A. R.; Clayton, R. A.; Sutton, G. G.; Fleischmann, R. D.; Ketchum, K. A.; Klenk, H. P.; Gill, S.; Dougherty, B. A.; Nelson, K.; Quackenbush, J.; Zhou, L.; Kirkness, E. F.; Peterson, S.; Loftus, B.; Richardson, D.; Dodson, R.; Khalak, H. G.; Glodek, A.; McKenney, K.; Fitzegerald, L. M.; Lee, N.; Adams, M. D.; Hickey, E. K.; Berg, D. E.; Gocayne, J. D.; Utterback, T. R.; Peterson, J. D.; Kelley, J. M.; Cotton, M. D.; Weidman, J. M.; Fujii, C.; Bowman, C.; Watthey, L.; Wallin, E.; Hayes, W. S.; Borodovsky, M.; Karp, P. D.; Smith, H. O.; Fraser, C. M.; Venter, J. C., The complete genome sequence of the gastric pathogen Helicobacter pylori. Nature 1997, 388 (6642), $539-47$.

78. Alm, R. A.; Ling, L. S.; Moir, D. T.; King, B. L.; Brown, E. D.; Doig, P. C.; Smith, D. R.; Noonan, B.; Guild, B. C.; deJonge, B. L.; Carmel, G.; Tummino, P. J.; Caruso, A.; Uria-Nickelsen, M.; Mills, D. M.; Ives, C.; Gibson, R.; Merberg, D.; Mills, S. D.; Jiang, Q.; Taylor, D. E.; Vovis, G. F.; Trust, T. J., Genomic-sequence 
comparison of two unrelated isolates of the human gastric pathogen Helicobacter pylori. Nature 1999, 397 (6715), 176-80.

79. Trust, T. J.; Alm, R. A.; Pappo, J., Helicobacter pylori: today's treatment, and possible future treatment. Eur J Surg Suppl 2001, (586), 82-8.

80. Salama, N. R.; Shepherd, B.; Falkow, S., Global transposon mutagenesis and essential gene analysis of Helicobacter pylori. J Bacteriol 2004, 186 (23), 7926-35.

81. Gavrish, E.; Shrestha, B.; Chen, C.; Lister, I.; North, E. J.; Yang, L.; Lee, R. E.; Han, A.; Williams, B.; Charnuska, D.; Coleman, K.; Lewis, K.; LaFleur, M. D., In vitro and in vivo activities of HPi1, a selective antimicrobial against Helicobacter pylori. Antimicrob Agents Chemother 2014, 58 (6), 3255-60.

82. Baldwin, D. N.; Shepherd, B.; Kraemer, P.; Hall, M. K.; Sycuro, L. K.; PintoSantini, D. M.; Salama, N. R., Identification of Helicobacter pylori genes that contribute to stomach colonization. Infect Immun 2007, 75 (2), 1005-16.

83. Kavermann, H.; Burns, B. P.; Angermuller, K.; Odenbreit, S.; Fischer, W.; Melchers, K.; Haas, R., Identification and characterization of Helicobacter pylori genes essential for gastric colonization. J Exp Med 2003, 197 (7), 813-22.

84. Chalker, A. F.; Minehart, H. W.; Hughes, N. J.; Koretke, K. K.; Lonetto, M. A.; Brinkman, K. K.; Warren, P. V.; Lupas, A.; Stanhope, M. J.; Brown, J. R.; Hoffman, P. S., Systematic identification of selective essential genes in Helicobacter pylori by genome prioritization and allelic replacement mutagenesis. $J$ Bacteriol 2001, 183 (4), 1259-68.

85. Katsura, Y.; Tomishi, T.; Inoue, Y.; Sakane, K.; Matsumoto, Y.; Morinaga, C.; Ishikawa, H.; Takasugi, H., Anti-Helicobacter pylori agents. 4. 2-(Substituted guanidino)-4-phenylthiazoles and some structurally rigid derivatives. J Med Chem 2000, 43 (17), 3315-21.

86. Katsura, Y.; Nishino, S.; Tomishi, T.; Sakane, K.; Matsumoto, Y.; Ishikawa, H.; Takasugi, H., Anti-Helicobacter pylori agents. 2. Structure activity relationships in a new series of 2-alkylguanidino-4-furylthiazoles. Bioorg Med Chem Lett 1998, 8 (11), 1307-12.

87. Katsura, Y.; Nishino, S.; Ohno, M.; Sakane, K.; Matsumoto, Y.; Morinaga, C.; Ishikawa, H.; Takasugi, H., Anti-Helicobacter pylori agents. 3. 2[(Arylalkyl)guanidino]-4-furylthiazoles. J Med Chem 1999, 42 (15), 2920-6.

88. Katsura, Y.; Tomishi, T.; Inoue, Y.; Sakane, K.; Matsumoto, Y.; Ishikawa, H.; Takasugi, H., Anti-Helicobacter pylori agents. 1. 2-(Alkylguanidino)-4furylthiazoles and related compounds. J Med Chem 1997, 40 (16), 2462-5. 89. Ishikawa, H.; Ito, H.; Higaki, M.; Higaki, M.; Matsumoto, Y.; Kamimura, T.; Katsura, Y.; Tomishi, T.; Inoue, Y.; Takasugi, H.; Tomoi, M.; Krakowka, S.; Yoshida, K., FR145715, a novel histamine H2 receptor antagonist, with specific anti-Helicobacter pylori activities. Eur J Pharmacol 1999, 378 (3), 299-310.

90. Katsura, Y.; Nishino, S.; Inoue, Y.; Sakane, K.; Matsumoto, Y.; Morinaga, C.; Ishikawa, H.; Takasugi, H., Anti-Helicobacter pylori agents. 5. 2-(Substituted guanidino)-4-arylthiazoles and aryloxazole analogues. J Med Chem 2002, 45 (1), 143-50.

91. Ando, R.; Kawamura, M.; Chiba, N., 3-(Arylacetylamino)-N-methylbenzamides: a novel class of selective anti-Helicobacter pylori agents. $J$ Med Chem 2001, 44 (25), 4468-74. 
92. Copeland, R. A.; Marcinkeviciene, J.; Haque, T. S.; Kopcho, L. M.; Jiang, W.; Wang, K.; Ecret, L. D.; Sizemore, C.; Amsler, K. A.; Foster, L.; Tadesse, S.; Combs, A. P.; Stern, A. M.; Trainor, G. L.; Slee, A.; Rogers, M. J.; Hobbs, F., Helicobacter pylori-selective antibacterials based on inhibition of pyrimidine biosynthesis. J Biol Chem 2000, 275 (43), 33373-8.

93. Marcinkeviciene, J.; Rogers, M. J.; Kopcho, L.; Jiang, W.; Wang, K.; Murphy, D. J.; Lippy, J.; Link, S.; Chung, T. D.; Hobbs, F.; Haque, T.; Trainor, G. L.; Slee, A.; Stern, A. M.; Copeland, R. A., Selective inhibition of bacterial dihydroorotate dehydrogenases by thiadiazolidinediones. Biochem Pharmacol 2000, 60 (3), 33942.

94. Haque, T. S.; Tadesse, S.; Marcinkeviciene, J.; Rogers, M. J.; Sizemore, C.; Kopcho, L. M.; Amsler, K.; Ecret, L. D.; Zhan, D. L.; Hobbs, F.; Slee, A.; Trainor, G. L.; Stern, A. M.; Copeland, R. A.; Combs, A. P., Parallel synthesis of potent, pyrazole-based inhibitors of Helicobacter pylori dihydroorotate dehydrogenase. $J$ Med Chem 2002, 45 (21), 4669-78.

95. Nakao, M.; Miyagaw, K.; Nakano, Y.; Sakane, T.; Tada, M.; Nishimura, O.; Fujino, M., Pyloricidins, novel anti-helicobacterpylori antibiotics produced by Bacillus sp. I. Taxonomy, fermentation and biological activity. J Antibiot (Tokyo) 2001, 54 (11), 926-33.

96. Nagano, Y.; Ikedo, K.; Fujishima, A.; Izawa, M.; Tsubotani, S.; Nishimura, O.; Fujino, M., Pyloricidins, novel anti-Helicobacter pylori antibiotics produced by bacillus sp. II. Isolation and structure elucidation. J Antibiot (Tokyo) 2001, 54 (11), 934-47.

97. Hasuoka, A.; Nishikimi, Y.; Nakayama, Y.; Kamiyama, K.; Nakao, M.; Miyagawa, K.; Nishimura, O.; Fujino, M., Synthesis and anti-Helicobacter pylori activity of pyloricidin derivatives II. The combination of amino acid residues in the dipeptidic moiety and its effect on the anti-Helicobacter pylori activity. $J$ Antibiot (Tokyo) 2002, 55 (5), 499-507.

98. Dekker, K. A.; Inagaki, T.; Gootz, T. D.; Kaneda, K.; Nomura, E.; Sakakibara, T.; Sakemi, S.; Sugie, Y.; Yamauchi, Y.; Yoshikawa, N.; Kojima, N., CJ-12,954 and its congeners, new anti-Helicobacter pylori compounds produced by Phanerochaete velutina: fermentation, isolation, structural elucidation and biological activities. $J$ Antibiot (Tokyo) 1997, 50 (10), 833-9.

99. Yano, K.; Yokoi, K.; Sato, J.; Oono, J.; Kouda, T.; Ogawa, Y.; Nakashima, T., Actinopyrones A, B and C, new physiologically active substances. II. Physicochemical properties and chemical structures. J Antibiot (Tokyo) 1986, 39 (1), 38-43.

100. Taniguchi, M.; Watanabe, M.; Nagai, K.; Suzumura, K.; Suzuki, K.; Tanaka, A., Gamma-pyrone compounds with selective and potent anti-Helicobacter pylori activity. J Antibiot (Tokyo) 2000, 53 (8), 844-7.

101. Kanamaru, T.; Nakano, Y.; Toyoda, Y.; Miyagawa, K. I.; Tada, M.; Kaisho, T.; Nakao, M., In vitro and in vivo antibacterial activities of TAK-083, an agent for treatment of Helicobacter pylori infection. Antimicrob Agents Chemother 2001, 45 (9), 2455-9.

102. Hasuoka, A.; Nakayama, Y.; Adachi, M.; Kamiguchi, H.; Kamiyama, K., Development of a stereoselective practical synthetic route to indolmycin, a candidate anti-H. pylori agent. Chem Pharm Bull (Tokyo) 2001, 49 (12), 1604-8. 
103. Preobrazhenskaya, M. N.; Balashova, E. G.; Turchin, K. F.; Padeiskaya, E. N.; Uvarova, N. V.; Pershin, G. N.; Suvorov, N. N., Total synthesis of antibiotic indolmycin and its stereoisomers. Tetrahedron 1968, 24 (19), 6131-43.

104. Sorba, G.; Bertinaria, M.; Di Stilo, A.; Gasco, A.; Scaltrito, M. M.; Brenciaglia, M. I.; Dubini, F., Anti-Helicobacter pylori agents endowed with H2-antagonist properties. Bioorg Med Chem Lett 2001, 11 (3), 403-6.

105. Carcanague, D.; Shue, Y. K.; Wuonola, M. A.; Uria-Nickelsen, M.; Joubran, C.; Abedi, J. K.; Jones, J.; Kuhler, T. C., Novel structures derived from 2-[[(2pyridyl)methyl]thio]-1H-benzimidazole as anti-Helicobacter pylori agents, Part 2. $J$ Med Chem 2002, 45 (19), 4300-9.

106. Mills, S. D.; Yang, W.; MacCormack, K., Molecular characterization of benzimidazole resistance in Helicobacter pylori. Antimicrob Agents Chemother 2004, 48 (7), 2524-30.

107. Berrisford, J. M.; Baradaran, R.; Sazanov, L. A., Structure of bacterial respiratory complex I. Biochim Biophys Acta 2016, 1857 (7), 892-901.

108. Degli Esposti, M., Inhibitors of NADH-ubiquinone reductase: an overview. Biochim Biophys Acta 1998, 1364 (2), 222-35.

109. Smith, M. A.; Finel, M.; Korolik, V.; Mendz, G. L., Characteristics of the aerobic respiratory chains of the microaerophiles Campylobacter jejuni and Helicobacter pylori. Arch Microbiol 2000, 174 (1-2), 1-10.

110. Lee, J. W.; Kim, N.; Nam, R.H.; Kim, J. M.; Park, J. Y.; Lee, S. M., Kim, J. S.; Lee, D. H.; Jung, H. C.; High efficacy of finafloxacin on helicobacter pylori isolates at pH 5.0 compared with that of other fluoroquinolones. Antimicrob Agents Chemother 2015, 59 (12), 7629-36.

111. Yamamoto, T.; Takano, T.; Higuchi, W.; Nishiyama, A.; Taneike, I.; Yoshida, K.; Kanda, H.; Imamura, Y.; Helicobacter pylori eradication by sitafloxacinlansoprazole combination and sitafloxacin pharmacokinetics in Mongolian gerbils and its in vitro activity and resistance development. Antimicrob Agents Chemother 2011, 55 (9), 4261-6.

112. Takano, T.; Higuchi, W.; Kanda, H.; Yamamoto, T.; In vitro activity of DX-619, a des-F(6)-quinolone, against Helicobacter pylori. J Antimicrob Chemother 2011, 66 (1), 220-2.

113. Blandizzi, C.; Malizia, T.; Gherardi, G.; Costa, F.; Marchi, S.; Marveggio, C.; Natale, G.; Senesi, S.; Bellini, M.; Maltinti, G.; Campa, M.; Tacca, M. D.; Gastric mucosal distribution and clinical efficacy of azithromycin in patients with helicobacter pylori related gastritis. J Antimicrob Chemother 1998, 42 (1), 75-82

114. Garcia-Rodriguez, J. A.; Garcia Sanchez, J. E.; Garcia Garcia, M. I.; Garcia Sanchez, E.; Munoz Bellido, J. L.; In vitro activities of new oral beta-lactams and macrolides against campylobacter pylori. Antimicrob Agents Chemother 1989, 33 (9), 1650-1

115. Goswami, S.; Bhakuni, R. S.; Chinniah, A.; Pal, A.; Kar, S. K.; Das, P. K.; Antihelicobacter pylori potential of artemisinin and its derivatives. Antimicrob Agents Chemother 2012, 56 (9), 4594-607.

116. Hoffman, P. S.; Sisson, G.; Croxen, M. A.; Welch, K.; Harman, W. D.; Cremades, N.; Morash, M. G.; Antiparasitic drug nitazoxanide inhibits the pyruvate 
oxidoreductases of helicobacter pylori, selected anaerobic bacteria and parasites, and campylobacter jejuni. Antimicrob Agents Chemother 2007, 51 (3), 868-76.

117. Tsutsui, N.; Taneike, I.; Ohara, T.; Goshi, S.; Kojio, S.; Iwakura, N.; Matsumaru, H.; Wakisaka-Saito, N.; Zhang, H. M.; Yamamoto, T.; A novel action of the proton pump inhibitor rabeprazole and its thioether derivative against the motility of helicobacter pylori. Antimicrob Agent Chemother 2000, 44 (11), 3069-73.

118. Nagata, K.; Takagi, E.; Tsuda, M.; Nakazawa, T.; Satoh, H.; Nakao, M.; Okamura, H.; Tamura, T.; Inhibitory action of lansoprazole and its analogs against helicobacter pylori: inhibition of growth is not related to inhibition of urease. Antimicrob Agents Chemother 1995, 39 (2), 567-70.

119. Inatsu, S.; Ohsaki, A.; Nagata, K.; Idebenone acts against growth of helicobacter pylori by inhibiting its respiration. Antimicrob Agents Chemother 2006, 50 (6), 2237-9.

120. Von Recklinghausen, G.; Di Maio, C.; Ansorq, R.; Activity of the antimycotic ketoconazole against helicobacter pylori. J Antimicrob Chemother 1992, 30 (2), 238-40.

121. Garcia Sanchez, E. J.; Garcia Saenz, N.; Rodriguez Rincon, M.; Trujillano Martin, I.; Garcia Sanchez, E.; Fresnadillo, M.; Susceptibility of helicobacter pylori to mupirocin, oxazolidinones, quinupristin/dalfopristin and new quinolones. $J$ Antimicrob Chemother 2000, 46 (2), 283-5.

122. Hirschl, A. M.; Apfalter, P.; Makristathis, A.;Rotter, M. L.; Rotter, M. L.; Wimmer, M.; In vitro activities of linezolid alone and in combination with amoxicillin, clarithromycin, and metronidazole against helicobacter pylori. Antimicrob Agents Chemother 2000, 44 (7), 1977-9.

123. Ansorq, R.; Von Recklinghausen, G.; Heintschel von Heinegg, E.; Susceptibility of helicobacter pylori to simethicone and other non-antibiotic drugs. $J$ Antimicrob Chemother 1996, 37 (1), 45-52.

124. Watanabe, A.; Tokue, Y.; Takahashi, H.; Kikuchi, T.; Kobayashi, T.; Gomi, K.; Fujimura, S.; Nukiwa, T.; In vitro activity of HSR-903, a new oral quinolone, against bacteria causing respiratory infections. Antimicrob Agents Chemother 1999, 43 (7), 1767-8.

125. Revelant, G.; Dunand, S.; Hesse, S.; Kirsch, G., Microwave-assisted synthesis of 5substituted 2- aminothiophenes starting from arylacetaldehydes. Synthesis 2011, 18, 2935-40

126. Ishikawa, F.; Kosasayama, A.; Yamaguchi, H.; Watanabe, Y.; Saegusa, J.; Shibamura, S.; Sakuma, K.; Ashida, S.; Abiko, Y., Cyclic guanidines. 14. Imidazo[1,2-a]thienopyrimidin-2-one derivatives as blood platelet aggregation inhibitors. J Med Chem 1981, 24 (4), 376-82.

127. Golub, A. G.; Bdzhola, V. G.; Briukhovetska, N. V.; Balanda, A. O.; Kukharenko, O. P.; Kotey, I. M.; Ostrynska, O. V.; Yarmoluk, S. M., Synthesis and biological evaluation of substituted (thieno[2,3-d]pyrimidin-4-ylthio)carboxylic acids as inhibitors of human protein kinase CK2. Eur J Med Chem 2011, 46 (3), 870-6.

128. Bugge, S.; Kaspersen, S. J.; Larsen, S.; Nonstad, U.; Bjorkoy, G.; Sundby, E.; Hoff, B. H., Structure-activity study leading to identification of a highly active thienopyrimidine based EGFR inhibitor. Eur J Med Chem 2014, 75, 354-74. 
129. Richter, M. F.; Drown, B. S.; Riley, A. P.; Garcia, A.; Shirai, T.; Svec, R. L.; Hergenrother, P. J.; Predictive compound accumulation rules yield a broadspectrum antibiotic. Nature 2017, 545 (7654), 299-304.

130. Rakesh; Bruhn, D.; Madhura, D. B.; Maddox, M.; Lee, R. B.; Trivedi, A.; Yang, L.; Scherman, M. S.; Gilliland, J. C.; Gruppo, V.; McNeil, M. R.; Lenaerts, A. J.; Meibohm, B.; Lee, R. E., Antitubercular nitrofuran isoxazolines with improved pharmacokinetic properties. Bioorg Med Chem 2012, 20 (20), 6063-72.

131. Di, L.; Kerns, E. H.; Li, S. Q.; Petusky, S. L., High throughput microsomal stability assay for insoluble compounds. Int $J$ Pharm 2006, 317 (1), 54-60.

132. Di, L.; Kerns, E. H.; Hong, Y.; Chen, H., Development and application of high throughput plasma stability assay for drug discovery. Int J Pharm 2005, 297 (1-2), $110-9$.

133. Lee, A.; O'Rourke, J.; De Ungria, M. C.; Robertson, B.; Daskalopoulos, G.; Dixon, M. F., A standardized mouse model of Helicobacter pylori infection: introducing the Sydney strain. Gastroenterology 1997, 112 (4), 1386-97.

134. Marchetti, M.; Arico, B.; Burroni, D.; Figura, N.; Rappuoli, R.; Ghiara, P., Development of a mouse model of Helicobacter pylori infection that mimics human disease. Science 1995, 267 (5204), 1655-8.

135. George, L. L.; Borody, T. J.; Andrews, P.; Devine, M.; Moore-Jones, D.; Walton, M.; Brandl, S., Cure of duodenal ulcer after eradication of Helicobacter pylori. Med J Aust 1990, 153 (3), 145-9.

136. Yagi, T.; Yano, T.; Di Bernardo, S.; Matsuno-Yagi, A., Procaryotic complex I (NDH-1), an overview. Biochim Biophys Acta 1998, 1364 (2), 125-33.

137. Yagi, T.; Matsuno-Yagi, A., The proton-translocating NADH-quinone oxidoreductase in the respiratory chain: the secret unlocked. Biochemistry 2003, 42 (8), 2266-74.

138. Sazanov, L. A., Respiratory complex I: mechanistic and structural insights provided by the crystal structure of the hydrophilic domain. Biochemistry 2007, 46 (9), 227588 .

139. Brandt, U., Energy converting NADH:quinone oxidoreductase (complex I). Annu Rev Biochem 2006, 75, 69-92.

140. Lee, A.; O'Rourke, J.; De Ungria, M. C.; Robertson, B.; Daskalopoulos, G.; Dixon, M. F., A standardized mouse model of Helicobacter pylori infection: introducing the Sydney strain. Gastroenterology 1997, 112 (4), 1386-97.

141. Bridges, H. R.; Jones, A. J.; Pollak, M. N.; Hirst, J., Effects of metformin and other biguanides on oxidative phosphorylation in mitochondria. Biochem J 2014, 462 (3), 475-87.

142. Molina, J. R.; Sun, Y.; Protopopova, M.; Gera, S.; Bandi, M.; Bristow, C.; McAfoos, T.; Morlacchi, P.; Ackroyd, J.; Agip, A. A.; Al-Atrash, G.; Asara, J.; Bardenhagen, J.; Carrillo, C. C.; Carroll, C.; Chang, E.; Ciurea, S.; Cross, J. B.; Czako, B.; Deem, A.; Daver, N.; de Groot, J. F.; Dong, J. W.; Feng, N.; Gao, G.; Gay, J.; Do, M. G.; Greer, J.; Giuliani, V.; Han, J.; Han, L.; Henry, V. K.; Hirst, J.; Huang, S.; Jiang, Y.; Kang, Z.; Khor, T.; Konoplev, S.; Lin, Y. H.; Liu, G.; Lodi, A.; Lofton, T.; Ma, H.; Mahendra, M.; Matre, P.; Mullinax, R.; Peoples, M.; Petrocchi, A.; Rodriguez-Canale, J.; Serreli, R.; Shi, T.; Smith, M.; Tabe, Y.; Theroff, J.; Tiziani, S.; Xu, Q.; Zhang, Q.; Muller, F.; DePinho, R. A.; Toniatti, C.; 
Draetta, G. F.; Heffernan, T. P.; Konopleva, M.; Jones, P.; Di Francesco, M. E.; Marszalek, J. R., An inhibitor of oxidative phosphorylation exploits cancer vulnerability. Nat Med 2018, 24 (7), 1036-1046.

143. Addie, M.; Ballard, P.; Buttar, D.; Crafter, C.; Currie, G.; Davies, B. R.; Debreczeni, J.; Dry, H.; Dudley, P.; Greenwood, R.; Johnson, P. D.; Kettle, J. G.; Lane, C.; Lamont, G.; Leach, A.; Luke, R. W.; Morris, J.; Ogilvie, D.; Page, K.; Pass, M.; Pearson, S.; Ruston, L., Discovery of 4-amino-N-[(1S)-1-(4-chlorophenyl)-3hydroxypropyl]-1-(7H-pyrrolo[2,3-d]pyrimidin -4-yl)piperidine-4-carboxamide (AZD5363), an orally bioavailable, potent inhibitor of Akt kinases. J Med Chem 2013, 56 (5), 2059-73.

144. Sen, S. E.; Roach, S. L.; A convenient two-step procedure for the synthesis of substituted allylic amines from allylic alcohols. Synthesis 1995, 7, 756-758.

145. Raub, T., Bursuhn, C., Williams, L., Decker, D., Sawada, G. and Ho, N. (2008) Use of A Biophysical-Kinetic Model to Understand Roles of Protein Binding and Membrane Partitioning On Passive Diffusion of Highly Lipophilic Molecules Across Cellular Barriers. J. Drug Target. 4, 269-286

146. Walker, A. M., (2014) Novel Tactics for Designing Water-Soluble Molecules in Drug Discovery. Expert Opin. Drug Discov. 9, 1421-1433

147. Smith, A.D., Beaumont, K., Maurer, S.T. and Di, L. (2015) Volume of Distrubution in Drug Design. J. Med Chem. 2015, 5691-5698

148. Bohm, H., Banner, D., Bendels, S., Kansy, M., Juhn, B., Muller, K., Obst-Sander, V. and Stahl, M. (2004) Fluorine in Medicinal Chemistry. ChemBioChem 5, 637-643

149. Bohm, H, Flohr, A. and Stahl, M. (2004) Scaffold Hopping. Drug Discov. Today 3, 217-224 


\section{APPENDIX.}

Table A-1. Inhibition profile of thienopyrimidines against $H$. pylori ATCC 43504 strain, SS1 strain and SS1 mutant strain.

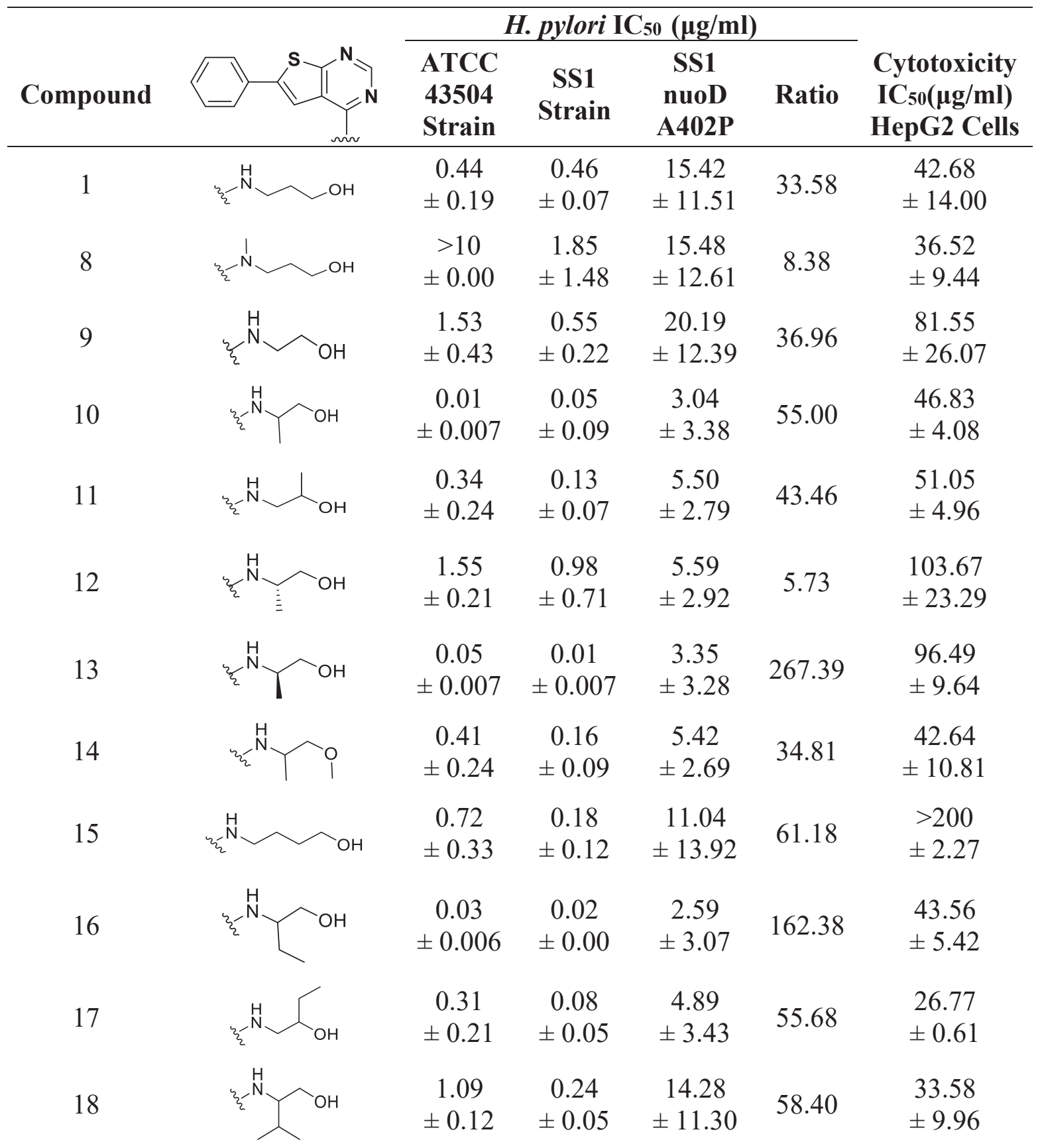


Table A-1. (Continued).

\begin{tabular}{|c|c|c|c|c|c|}
\hline \multirow[b]{2}{*}{ Compound } & \multicolumn{4}{|c|}{ H. pylori $\mathrm{IC}_{50}(\mu \mathrm{g} / \mathrm{ml})$} & \multirow[b]{2}{*}{$\begin{array}{c}\text { Cytotoxicity } \\
\text { IC } 50(\mu \mathrm{g} / \mathrm{ml}) \\
\text { HepG2 } \\
\text { Cells }\end{array}$} \\
\hline & $\begin{array}{c}\text { ATCC } \\
43504 \\
\text { Strain }\end{array}$ & $\begin{array}{c}\text { SS1 } \\
\text { Strain }\end{array}$ & $\begin{array}{c}\text { SS1 } \\
\text { nuoD } \\
\text { A402P }\end{array}$ & Ratio & \\
\hline 19 & $\begin{array}{c}0.08 \\
\pm 0.06\end{array}$ & $\begin{array}{c}0.02 \\
\pm 0.002\end{array}$ & $\begin{array}{c}0.29 \\
\pm 0.33\end{array}$ & 17.68 & $\begin{array}{l}>200 \\
\pm 0.00\end{array}$ \\
\hline 20 & $\begin{aligned} & 0.03 \\
\pm & 0.004\end{aligned}$ & $\begin{array}{c}0.22 \\
\pm 0.17\end{array}$ & $\begin{array}{c}3.34 \\
\pm 3.41\end{array}$ & 15.36 & $\begin{array}{c}135.01 \\
\pm 40.25\end{array}$ \\
\hline 21 & $\begin{array}{c}0.92 \\
\pm 0.30\end{array}$ & $\begin{array}{c}0.48 \\
\pm 0.32\end{array}$ & $\begin{array}{c}1.27 \\
\pm 1.87\end{array}$ & 2.63 & $\begin{array}{l}29.26 \\
\pm 3.34\end{array}$ \\
\hline 22 & $\begin{array}{c}1.97 \\
\pm 0.71\end{array}$ & $\begin{array}{c}1.08 \\
\pm 0.47\end{array}$ & $\begin{array}{c}4.4 \\
\pm 2.98\end{array}$ & 4.07 & $\begin{array}{l}31.86 \\
\pm 3.38\end{array}$ \\
\hline 23 & $\begin{array}{c}0.57 \\
\pm 0.08\end{array}$ & $\begin{array}{c}0.24 \\
\pm 0.17\end{array}$ & $\begin{array}{c}5.48 \\
\pm 2.83\end{array}$ & 23.13 & $\begin{array}{c}12.45 \\
\pm 1.70\end{array}$ \\
\hline 24 & $\begin{array}{c}0.06 \\
\pm 0.03\end{array}$ & $\begin{array}{c}0.09 \\
\pm 0.03\end{array}$ & $\begin{array}{c}3.53 \\
\pm 0.29\end{array}$ & 41.12 & $\begin{array}{c}6.51 \\
\pm 11.515\end{array}$ \\
\hline 25 & $\begin{array}{c}0.004 \\
\pm 0.000\end{array}$ & $\begin{array}{c}0.004 \\
\pm 0.000\end{array}$ & $\begin{array}{c}0.1 \\
\pm 0.09\end{array}$ & 18.41 & $\begin{array}{l}>200 \\
\pm 0.00\end{array}$ \\
\hline 26 & $\begin{array}{c}0.004 \\
\pm 0.002\end{array}$ & $\begin{array}{c}0.004 \\
\pm 0.000\end{array}$ & $\begin{array}{c}0.06 \\
\pm 0.05\end{array}$ & 15.59 & $\begin{array}{l}18.76 \\
\pm 3.64\end{array}$ \\
\hline 27 & $\begin{array}{c}0.02 \\
\pm 0.003\end{array}$ & $\begin{array}{c}0.05 \\
\pm 0.045\end{array}$ & $\begin{array}{c}1.75 \\
\pm 0.71\end{array}$ & 45.55 & $\begin{array}{l}28.22 \\
\pm 0.54\end{array}$ \\
\hline 28 & $\begin{array}{c}0.02 \\
\pm 0.003\end{array}$ & $\begin{array}{c}0.01 \\
\pm 0.007\end{array}$ & $\begin{array}{c}0.44 \\
\pm 0.31\end{array}$ & 39.11 & $\begin{array}{l}<6.25 \\
\pm 0.00\end{array}$ \\
\hline
\end{tabular}


Table A-1. (Continued).

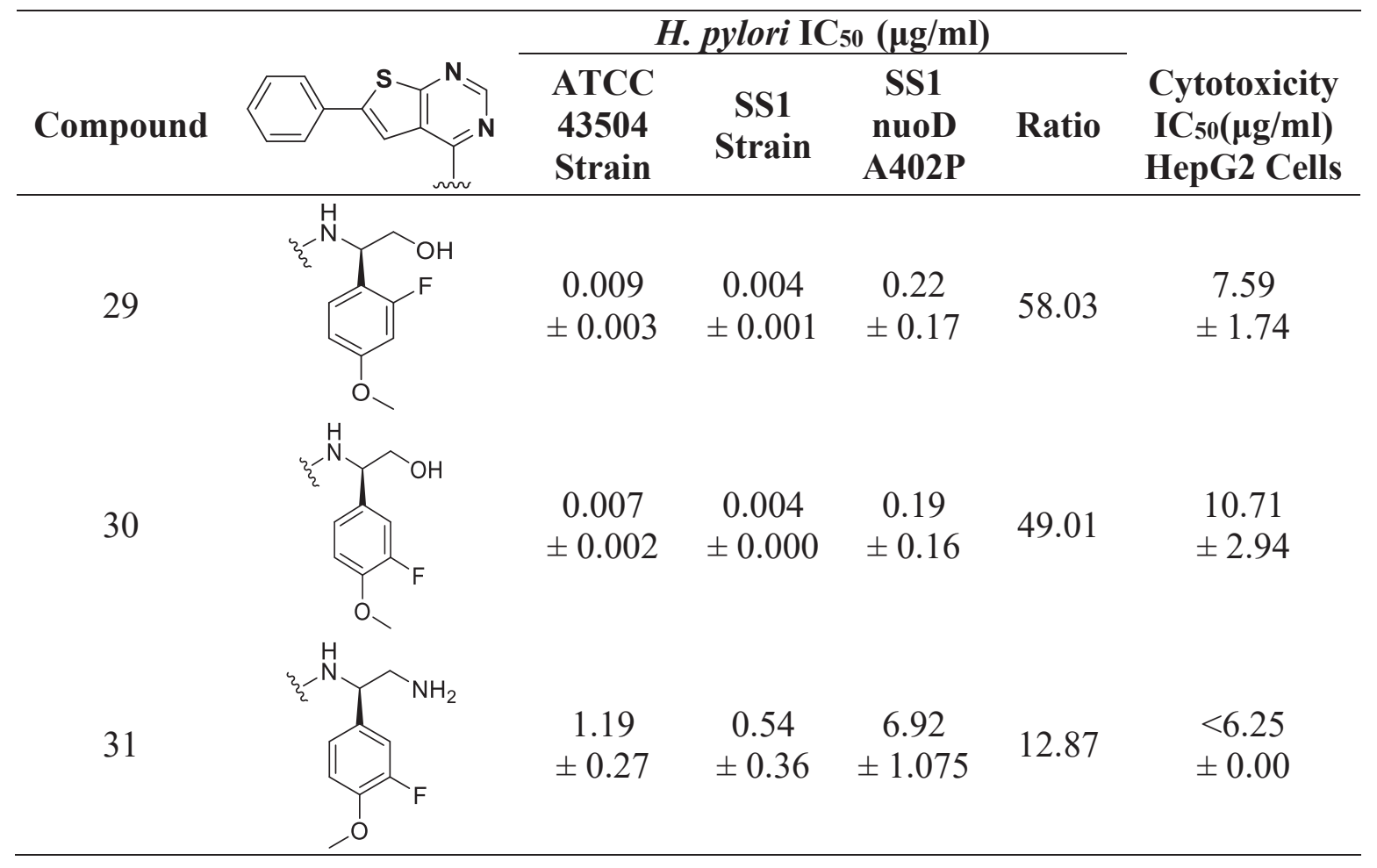


Table A-2. Inhibition profile of thienopyrimidines with modifications at the 5 and 6 positions against $H$. pylori ATCC 43504 strain, SS1 strain and SS1 mutant strain.

\begin{tabular}{|c|c|c|c|c|c|c|c|}
\hline \multirow[b]{2}{*}{ Compound } & \multirow[b]{2}{*}{$\mathbf{R}_{\mathbf{1}}$} & \multirow[b]{2}{*}{$\mathbf{R}_{2}$} & \multicolumn{4}{|c|}{ H. pylori $\mathrm{IC}_{50}(\mu \mathrm{g} / \mathrm{ml})$} & \multirow[b]{2}{*}{$\begin{array}{l}\text { Cytotoxicity } \\
\text { IC50 }(\mu \mathrm{g} / \mathrm{ml}) \\
\text { HepG2 Cells }\end{array}$} \\
\hline & & & $\begin{array}{c}\text { ATCC } \\
43504 \\
\text { Strain }\end{array}$ & $\begin{array}{c}\text { SS1 } \\
\text { Strain }\end{array}$ & $\begin{array}{c}\text { SS1 } \\
\text { nuoD } \\
\text { A402P }\end{array}$ & Ratio & \\
\hline 37 & $\mathrm{CH}_{3}$ & $\mathrm{CH}_{3}$ & $\begin{array}{c}1.77 \\
\pm 0.05\end{array}$ & $\begin{array}{c}0.25 \\
\pm 0.18\end{array}$ & $\begin{array}{c}5.05 \\
\pm 3.26\end{array}$ & 19.89 & $\begin{array}{l}<6.25 \\
\pm 0.00\end{array}$ \\
\hline 38 & $\mathrm{CH}_{3}$ & & $\begin{array}{c}0.12 \\
\pm \\
0.007\end{array}$ & $\begin{array}{c}0.02 \\
\pm 0.001\end{array}$ & $\begin{array}{c}3.71 \\
\pm 2.87\end{array}$ & 222.93 & $\begin{array}{r}20.64 \\
\pm 6.00\end{array}$ \\
\hline 39 & & $\mathrm{CH}_{3}$ & $\begin{array}{c}1.51 \\
\pm 1.49\end{array}$ & $\begin{array}{c}10.24 \\
\pm 13.58\end{array}$ & $\begin{array}{c}10.51 \\
\pm 12.95\end{array}$ & 1.03 & $\begin{array}{r}32.93 \\
\pm 1.92\end{array}$ \\
\hline 40 & & $\mathrm{H}$ & $\begin{array}{l}1.51 \\
\pm 0.27\end{array}$ & $\begin{array}{c}0.215 \\
\pm 0.16\end{array}$ & $\begin{array}{c}0.44 \\
\pm 0.35\end{array}$ & 2.06 & $\begin{array}{r}27.86 \\
\pm 9.41\end{array}$ \\
\hline
\end{tabular}

Table A-3. ADME of the lead compound 25.

\begin{tabular}{|c|c|c|c|c|c|c|}
\hline Compound & MW & $\mathrm{Clog} P$ & $\begin{array}{c}\text { Solubility } \\
{[\boldsymbol{[ \mu \mathrm { M } ]}}\end{array}$ & $\begin{array}{c}\text { PAMPA }^{a} \\
\text { Avg Pe } \\
\left(10^{-6}\right. \\
\mathrm{cm} / \mathrm{s})\end{array}$ & $\begin{array}{c}\text { Mouse } \\
\text { Plasma } \\
\text { Binding } \\
(\%)\end{array}$ & 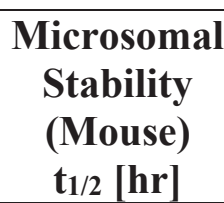 \\
\hline 25 & 377 & 4.23 & $\begin{array}{c}1 \\
\pm 0.2\end{array}$ & $\begin{array}{c}239.5 \\
\pm 180.5\end{array}$ & $\begin{array}{l}99.3 \\
\pm 0.1\end{array}$ & $\begin{array}{c}3.8 \\
\pm 0.37\end{array}$ \\
\hline
\end{tabular}

${ }^{a}$ The PAMPA assay was carried out at physiological $(\mathrm{pH}=7.4)$. 
Table A-4. Evaluation of in vivo pharmacokinetic properties of the lead compound 25.

\begin{tabular}{ccccccccc}
\hline Compound & Route & $\begin{array}{c}\text { Dose } \\
{[\mathbf{m g} / \mathbf{k g}]}\end{array}$ & $\begin{array}{c}\mathbf{T}_{\max } \\
(\mathbf{h r})\end{array}$ & $\begin{array}{c}\mathbf{C}_{\max } \\
(\mathbf{n g} / \mathbf{m L})\end{array}$ & $\begin{array}{c}\mathbf{A U} \mathbf{C}_{\text {last }} \\
\left(\mathbf{h r} \mathbf{n}^{*} \mathbf{n g} / \mathbf{m L}\right)\end{array}$ & $\begin{array}{c}\mathbf{T}_{\mathbf{1} / 2} \\
(\mathbf{h r})\end{array}$ & $\begin{array}{c}\mathbf{C L} \\
(\mathbf{m L} / \mathbf{m i n} / \mathbf{k g})\end{array}$ & $\begin{array}{c}\text { Vss } \\
(\mathbf{L} / \mathbf{k g})\end{array}$ \\
\hline \multirow{2}{*}{25} & i.p. & 50 & 2.00 & 2550.25 & 31907.71 & 2.79 & 26.05 & 6.28 \\
& PO & 10 & 0.25 & 589.84 & 674.42 & 1.52 & - & - \\
\hline
\end{tabular}

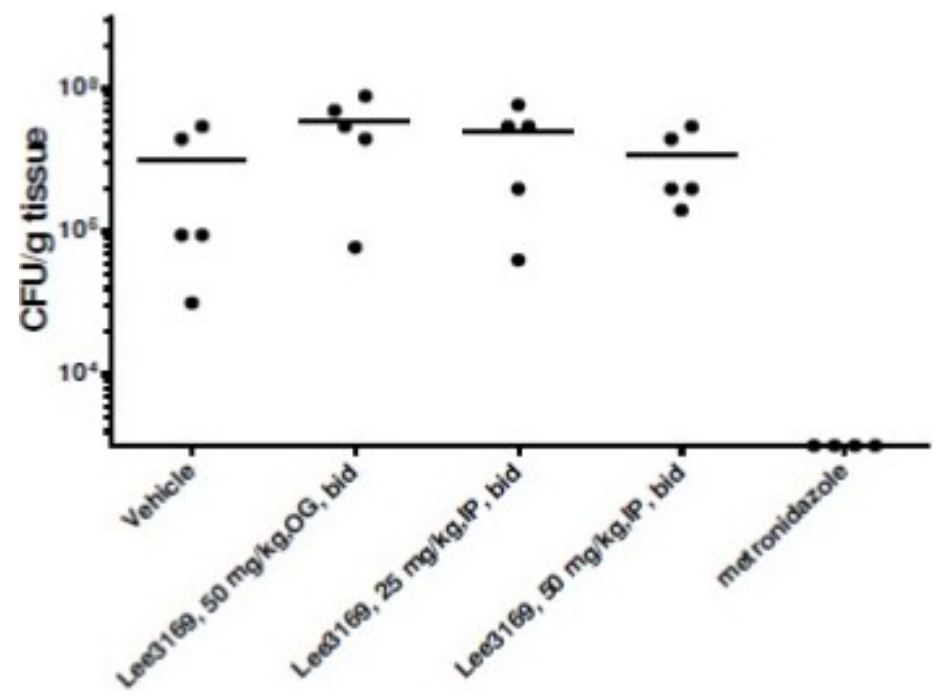

Figure A-1. In vivo efficacy of compound 25 in the $\mathrm{H}$. pylori infected mouse model. C57BL16 mice were infected with a H. pylori SS1 strain suspension. Then, mice were treated with metronidazole as control, and the following doses of compound $\mathbf{2 5}$, twice per day: $50 \mathrm{mg} / \mathrm{kg}(\mathrm{OG}), 50 \mathrm{mg} / \mathrm{kg}$ (IP) and $25 \mathrm{mg} / \mathrm{kg}$ (IP). 


\section{VITA}

Alex K. Mugengana was born in Mwanza, Tanzania in 1988. He received a bachelor's degree in Chemistry from Philander Smith College in Little Rock, Arkansas. After he graduated from college, he trained as an intern in the laboratory of Dr. William Tolleson at the National Center for Toxicological Research/ Food and Drug Administration (NCTR/FDA) in Jefferson, Arkansas. In 2013, he joined the pharmaceutical sciences graduate program at UTHSC to pursue his doctoral studies. In the following year, he served as vice-president in the Graduate Student Executive Committee. He is a recipient of the Honor Medal from the Office of International Program/ FDA and was recognized at the Black Student Association (BSA) Annual Awards Banquet as the 2016 College of Graduate Health Science (CGHS) Outstanding Student. In December of 2019, he will graduate with a Doctor of Philosophy degree. 